\title{
Calculation of thermal expansion of iron -aluminides with transition metal additives
}

Tatiana Seletskaia

West Virginia University

Follow this and additional works at: https://researchrepository.wvu.edu/etd

\section{Recommended Citation}

Seletskaia, Tatiana, "Calculation of thermal expansion of iron -aluminides with transition metal additives" (2002). Graduate Theses, Dissertations, and Problem Reports. 1698.

https://researchrepository.wvu.edu/etd/1698

This Dissertation is protected by copyright and/or related rights. It has been brought to you by the The Research Repository @ WVU with permission from the rights-holder(s). You are free to use this Dissertation in any way that is permitted by the copyright and related rights legislation that applies to your use. For other uses you must obtain permission from the rights-holder(s) directly, unless additional rights are indicated by a Creative Commons license in the record and/ or on the work itself. This Dissertation has been accepted for inclusion in WVU Graduate Theses, Dissertations, and Problem Reports collection by an authorized administrator of The Research Repository @ WVU.

For more information, please contact researchrepository@mail.wvu.edu. 


\title{
Calculation of Thermal Expansion of Iron-Aluminides with Transition Metal Additives
}

\author{
Tatiana Seletskaia
}

Dissertation submitted to the

Eberly College of Arts and Sciences at West Virginia University

in partial fulfillment of the requirements for the Degree of

Doctor of Philosophy in Physics

Bernard R. Cooper, Ph.D., Chair

Martina E. Bachlechner, Ph.D.

Mohindar S. Seehra, Ph.D.

David Lederman, Ph.D.

Bruce Kang, Ph.D.

Department of Physics

Morgantown, West Virginia

2002

Keywords: Iron-aluminides, Thermal expansion, Site selection 


\title{
ABSTRACT
}

\section{Calculation of Thermal Expansion of Iron-Aluminides}

\author{
with Transition Metal Additives
}

\author{
Tatiana Seletskaia
}

The addition of transition metal elements can significantly modify physical properties of intermetalic compounds. We studied the influence of Mo and $\mathrm{V}$ additives on thermal expansion coefficient (CTE) of $\mathrm{Fe}_{3} \mathrm{Al}$ and $\mathrm{FeAl}$ over the wide range of temperatures. The site preference of both transition metals was determined by FP-LMTO method. $\mathrm{Fe}_{3} \mathrm{Al}$ in $\mathrm{DO}_{3}$ structure has three non-equivalent lattice sites: $\mathrm{Fe}_{\mathrm{I}}$ site surrounded by eight iron atoms, $\mathrm{Fe}_{\mathrm{II}}$ site with four iron and four aluminum nearest neighbors, and $\mathrm{Al}$ site. Our calculations show that $\mathrm{Fe}_{\mathrm{I}}$ site is energetically more favorable for both Mo and $\mathrm{V}$. The obtained site-selection energies of Mo are relatively small, while for $\mathrm{V}$ they are much larger indicating that this additive is likely to improve the stability of $\mathrm{Fe}_{3} \mathrm{Al} \mathrm{DO} \mathrm{DO}_{3}$ structure. Thermal expansion of pure $\mathrm{FeAl}$ and $\mathrm{Fe}_{3} \mathrm{Al}$ was found directly from FP-LMTO calculations by incorporating them into the Debye model of a solid. The obtained results are within $10 \%$ of the experimentally measured values. To test the applicability of the Debye model to these compounds, we performed Molecular Dynamics (MD) simulation based on our many-body atomistic potentials. The potential parameters were fitted to reproduce the total energy of a crystal under various types of deformations obtained by FP-LMTO method and were tested with respect to different structures and vacancy formation energies. For pure iron-aluminides, thermal expansion calculated within the Debye model differs from the results of MD only at high temperatures. However, when the additives are present, the Debye model does not provide the correct description of thermal expansion. Our calculations show that the addition of $\mathrm{V}$ decreases the CTEs of both iron-aluminides, while the addition of Mo makes $\mathrm{Fe}_{3} \mathrm{Al} \mathrm{DO}_{3}$ structure unstable. 


\section{Acknowledgements}

I would like to express my sincerest gratitude and thanks to Professor Cooper for his input and supervision as a head of my graduate committee.

I also would like to thank all my committee members for their time and effort dedicated to helping me succeed.

Finally, I would like to thank the members of Professor Copper's research group and particularly Leonid Muratov for their helpful discussions. 


\section{TABLE OF CONTENTS}

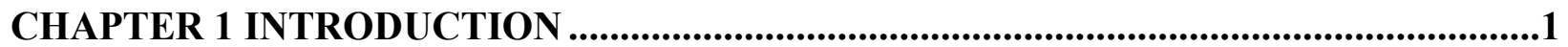

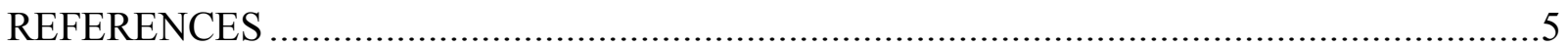

CHAPTER 2 ........................................................................................................6

FULL-POTENTIAL LINEAR MUFFIN-TIN ORBITAL METHOD (FP-LMTO) ..............6

2.1 BASIS OF ONE-ELECTRON LOCAL POTENTIAL THEORY ..................................6

2.2 DENSITY FUNCTIONAL THEORY (DFT) .........................................................

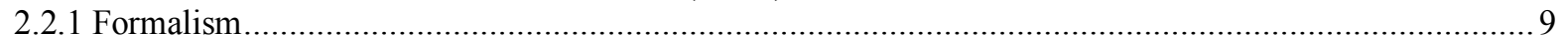

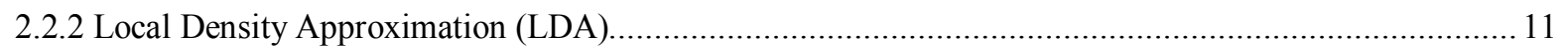

2.3 FULL-POTENTIAL LINEAR MUFFIN-TIN ORBITAL METHOD (FP-LMTO) ...........13

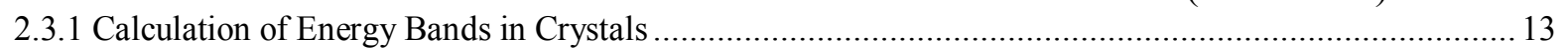

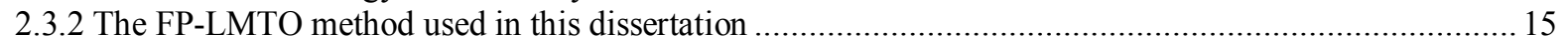

REFERENCES ...............................................................................................23

CHAPTER 3 SITE PREFERENCE OF MO AND V ADDITIVES IN FE 3 AL .................25

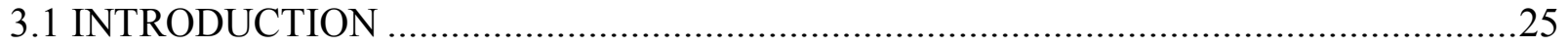

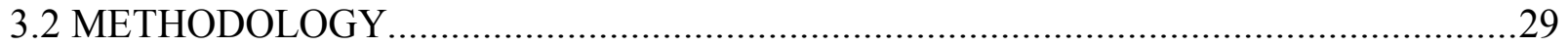

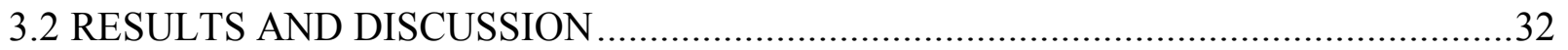

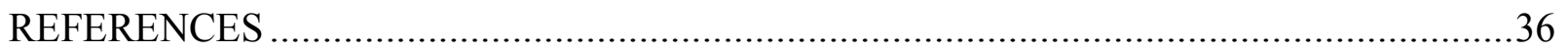

APPENDIX: THE INPUT DATA FILE FOR OUR FP-LMTO PROGRAM........................37

CHAPTER 4 FP-LMTO CALCULATION OF THERMAL EXPANSION........................43

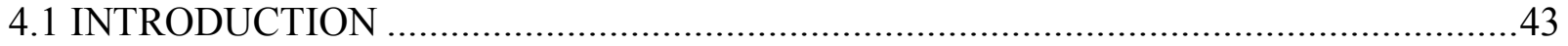

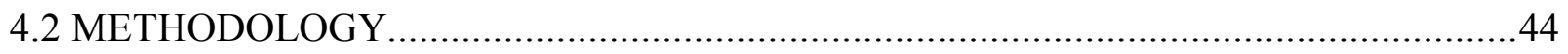

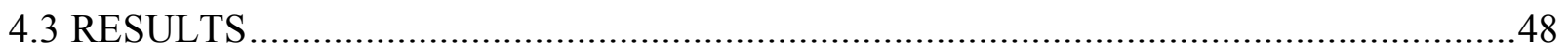

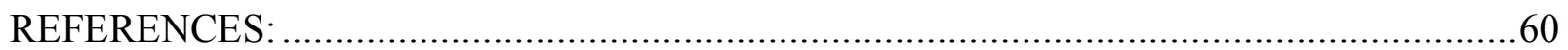

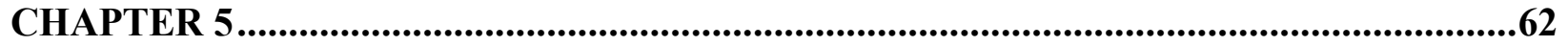

MOLECULAR DYNAMICS SIMULATION OF THERMAL EXPANSION OF IRONALUMINIDES IN THE PRESENCE OF ADDITIVES ................................................62

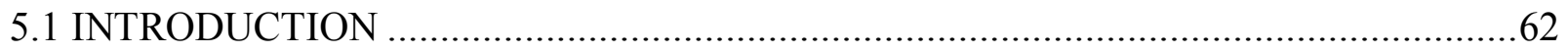

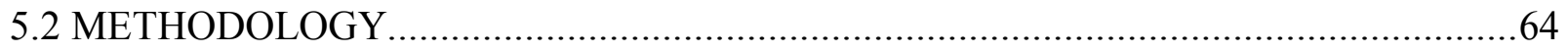

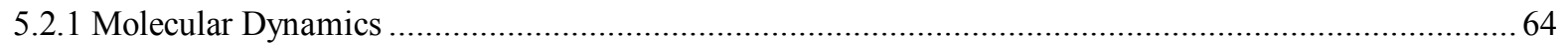

5.2.2 Many-body atomistic potentials for iron-aluminides with the addition of transition metals ..................... 70

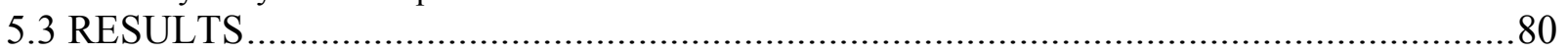

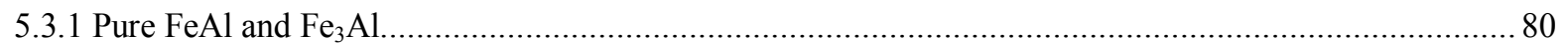

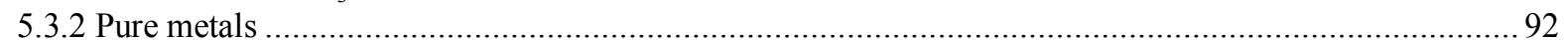

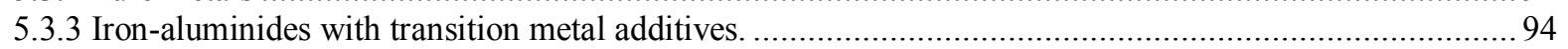

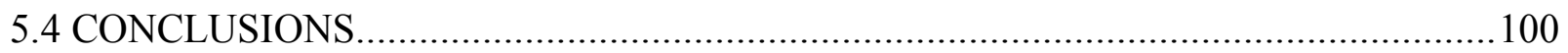

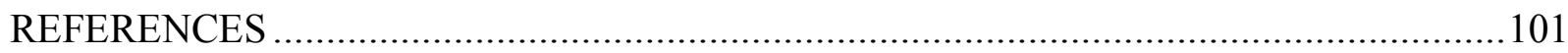




\section{List Of Tables}

Table 3.1 Effective formation energies of various defects in $\mathrm{Fe}_{3} \mathrm{Al}$

Table 4.1 Equilibrium lattice constants and elastic constants of $\mathrm{FeAl}$ and $\mathrm{Fe}_{3} \mathrm{Al}$.

Table 4.2 Elastic constants of $\mathrm{FeAl}$ and $\mathrm{Fe}_{3} \mathrm{Al}$ calculated at the experimental lattice constants

Table 4.3 Calculated third-order elastic constants in GPa.

Table 4.4 Elastic constants of $\mathrm{Fe}_{3} \mathrm{Al}$ with the addition of Mo and $\mathrm{V}$

Table 4.5 Calculated third-order elastic constants in $\mathrm{GPa}$ of $\mathrm{Fe}_{3} \mathrm{Al}$ with $\mathrm{Mo}$ and $\mathrm{V}$ additives.

Table 5.1 Cutoff distances in A for different atomic types

Table 5.2 Atomic density parameters found from the fitting of the relationship given by (5.12).

Table 5.3 Potential parameters for lattice expansion energy curve (5.17), for bcc structure.

Table 5.4 Pair-potential parameters for expression given by (5.18), found by fitting FPLMTO total energies for the compound with the lattice under deformations.

Table 5.5 Three-body interaction parameters for use in the expression given by (5.19) found by fitting FP-LMTO total energies for distorted lattices.

Table 5.6 Lattice vectors and basis of the selected lattice structures. The vectors are represented in Cartesian coordinates.

Table 5.7 Computed structural data for $\mathrm{FeAl}$ and $\mathrm{Fe}_{3} \mathrm{Al}$ within many-body atomistic potentials, ab initio calculations and semi-empirical method of Besson et al.

Table 5.8 Point defect energies $(\mathrm{eV})$ for $\mathrm{B}_{2}$ FeAl. The subscript $v$ stands for vacancy, the subscript $a$ is used for anti-site defect.

Table 5.9 Point defect energies (eV) for $\mathrm{DO}_{3} \mathrm{Fe}_{3} \mathrm{Al}$

Table 5.10 Elastic constants in $\mathrm{GPa}$ of $\mathrm{FeAl}$ and $\mathrm{Fe}_{3} \mathrm{Al}$ at different temperatures 


\section{List Of Figures}

1.1 Iron-aluminides phase diagram

1.2 $\mathrm{B}_{2}$ structure of FeAl

1.3 $\mathrm{DO}_{3}$ structure of $\mathrm{Fe}_{3} \mathrm{Al}$

2.1 $\mathrm{DO}_{3}$ structure of $\mathrm{Fe}_{3} \mathrm{Al}$

2.2 Electronic band structure of $\mathrm{Fe}_{3} \mathrm{Al}$ obtained by FP-LMTO method

4.1 Equation of state for $\mathrm{Fe}_{3} \mathrm{Al}$

4.2 Calculated bulk modulus as a function of lattice parameter for $\mathrm{Fe}_{3} \mathrm{Al}$

4.3. Temperature dependence of the linear thermal expansion of $\mathrm{FeAl}$ and $\mathrm{Fe}_{3} \mathrm{Al}$

4.4 The effect of the Mo and $\mathrm{V}$ additives on the thermal expansion of $\mathrm{Fe}_{3} \mathrm{Al}$

5.1 Divergence of trajectories in molecular dynamics

5.2 Energy conservation during molecular dynamics

5.3 (a, b) The results of the fitting procedure

5.4 Distance-dependence of pair-potentials given by 5.17

5.5 Distance-dependence of 3-body term given by 5.19

5.6 (a, b) The Brilloin zones of FeAl B $\mathrm{B}_{2}$ and $\mathrm{Fe}_{3} \mathrm{Al} \mathrm{DO}_{3}$ structures with the special k-points

5.7 (a, b) The phonon frequencies of $\mathrm{FeAl}$ ( on the left-hand side) and of $\mathrm{Fe}_{3} \mathrm{Al}$ (on the righthand side)

5.8 Linear Thermal Expansion of FeAl

5.9 Linear Thermal Expansion of $\mathrm{Fe}_{3} \mathrm{Al}$

5.10 (a, b, c and d) Linear Thermal Expansion of Fe, Al, Mo and V

5.11 Linear Thermal Expansion of $\left(\mathrm{Fe}_{1-\mathrm{x}} \mathrm{V}_{\mathrm{x}}\right)_{3} \mathrm{Al}$ pseudo-binary alloy $(x=8.3 \%)$.

5.12 Calculated phonon density of states of $\mathrm{Fe}_{3} \mathrm{Al}$ (black line) and $\left(\mathrm{Fe}_{1-\mathrm{x}} \mathrm{V}_{\mathrm{x}}\right)_{3} \mathrm{Al}$ with $x=8.3 \%$ (redline)

5.13 CTE of $\mathrm{Fe}_{1-\mathrm{x}} \mathrm{V}_{\mathrm{x}} \mathrm{Al}$ pseudo-binary alloys

5.14 CTE of $\left(\mathrm{Fe}_{1-\mathrm{x}} \mathrm{V}_{\mathrm{x}}\right)_{3} \mathrm{Al}$ pseudo-binary alloys

5.15 CTE of $\left(\mathrm{Fe}_{1-\mathrm{x}} \mathrm{Mo}_{\mathrm{x}}\right)_{3} \mathrm{Al}$ pseudo-binary alloys 


\section{Chapter 1 Introduction}

The iron-aluminides based on $\mathrm{Fe}_{3} \mathrm{Al}$ and $\mathrm{FeAl}$ possess unique properties and have development potential as new materials for structural use [1]. In addition to excellent oxidation and corrosion resistance, the aluminides offer low cost and low density. However, the major drawbacks of the aluminides are their low ductility at ambient temperature and poor strength at the temperatures above $600 \mathrm{C}$. Recently, a lot of effort has been put toward improvement of these properties through the control of material processing as well as alloying the aluminides with the transition-metal additives.

The low ductility of iron-aluminides is particularly referred to the strong covalent bonding that aluminum tends to form with the iron atoms. The embrittlement of FeAl compound has environmental nature [2], therefore, it improves very little with the addition of alloying components such as Boron. Even though, the problem of FeAl embrittlement remains an open question, the ductility of $\mathrm{Fe}_{3} \mathrm{Al}$ can be sufficiently increased by the proper control of the processing and the addition of Chromium from 2 to $6 \%$ of the total composition to this compound $[3,4]$.

The drop in strength of $\mathrm{Fe}_{3} \mathrm{Al}$ above $600 \mathrm{C}$ occurs due to the second-order phase transition from ordered $\mathrm{DO}_{3}$ structure to disordered $\mathrm{B}_{2} \mathrm{FeAl}$ phase [5] demonstrated in the phase diagram, Figure 1.1. $\mathrm{B}_{2}$ and $\mathrm{DO}_{3}$ structures are shown in Figures 1.2 and 1.3, respectively. 
Figure 1.1 Iron-aluminides phase diagram.

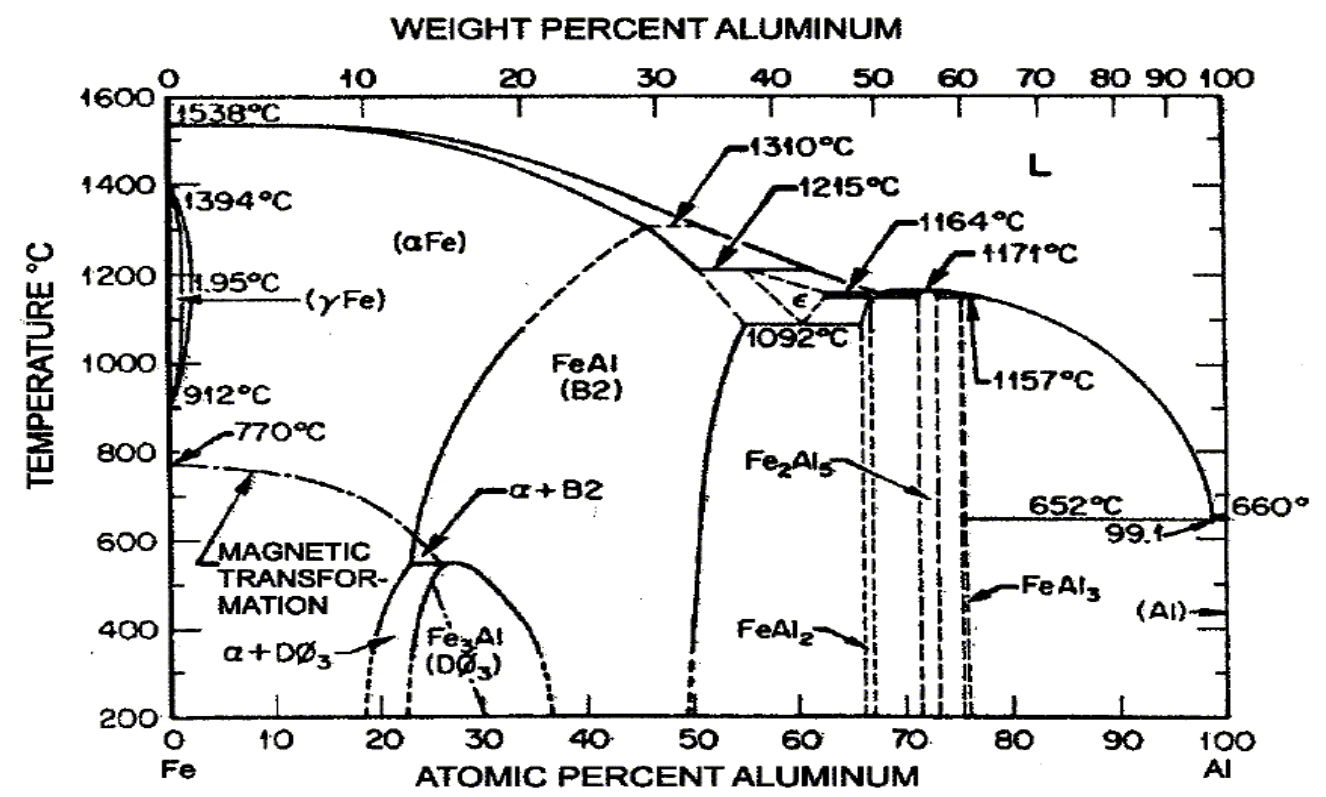

Figure 1.2 $\mathrm{B}_{2}$ structure of FeAl. Fe sites are shown by transparent circles and Al sites are represented by black-filled circles.

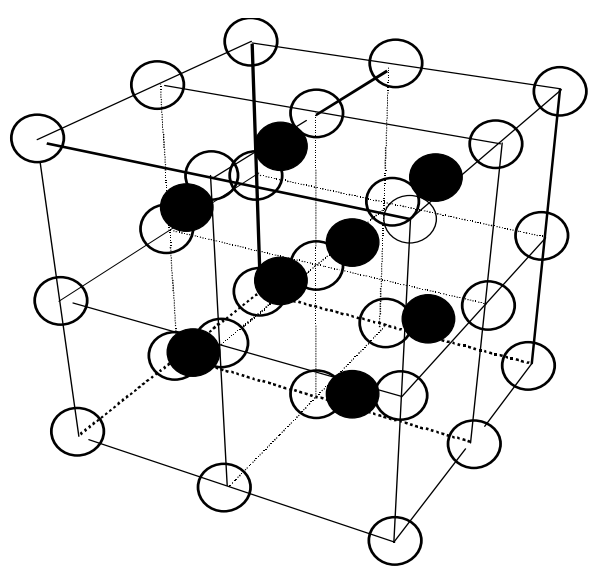

Figure 1.3 $\mathrm{DO}_{3}$ structure of $\mathrm{Fe}_{3} \mathrm{Al}$. It has three non-equivalent lattice sites: FeI sites (crosshatched circles) surrounded by eight iron atoms, FeII sites (transparent circles) with four iron and four aluminum nextneighbor, and the rest are Al sites (black-filled circles).

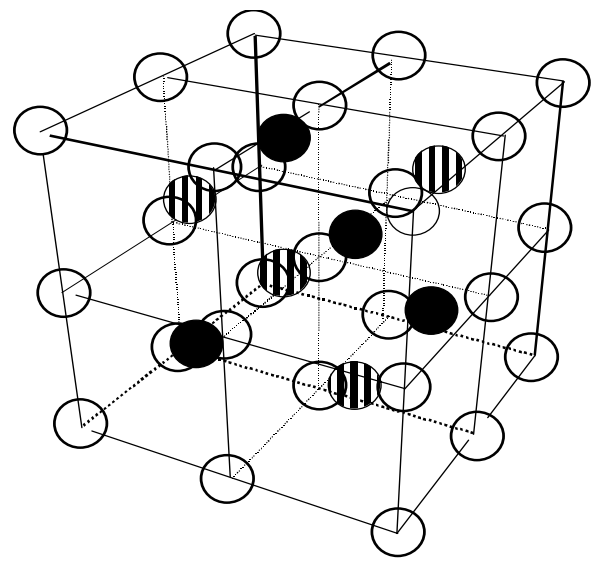


Recent experiments indicated that addition of $3 d$ transition elements, such as Mo, $\mathrm{V}$ and Ti leads to remarkable stabilization of $\mathrm{DO}_{3}$ structure and thus improves the strength of $\mathrm{Fe}_{3} \mathrm{Al}$ [6]. At the same time it was observed that the addition of Mo improves the creep resistance in $\mathrm{Fe}_{3} \mathrm{Al}$ [7]. Therefore, there is a natural interest in studying the effect of transition-metal additives on the physical properties of iron-aluminides.

One of the most promising applications of the aluminides is their use as the coating materials at high temperatures. There are many situations when the thermal expansion can cause the appearance of the macro cracks in the coating. Anisotropy of the thermal expansion can lead to the uneven deformation of the coating in different directions [8]. A similar effect can be produced in the case when the thermal expansion of the coating strongly differs from that of the material in a contact. Thus, the thermal expansion becomes one of the important characteristics of the aluminides that has to be studied. We also have to mention that conducting the measurements of the thermal expansion coefficient is by no means a trivial task. It is hard to grow a single crystal of these intermetallics and the measurements have to be carried at the temperatures appropriate for their future use. So far no experiments were done on studying how the transition metal additives are changing the coefficient of thermal expansion. The intent of our computational research is to compensate for the lack of this empirical knowledge. Here we briefly describe the composition of this dissertation.

Before simulating any thermal or mechanical process in the intermetallic compounds in the presence of additives, it is necessary to determine the probability of each site to be occupied by the additive. To solve the problem of site preference of transition metals $\mathrm{Mo}$ and $\mathrm{V}$ in $\mathrm{Fe}_{3} \mathrm{Al}$, we employed our full-potential Linearized Muffin-Tin Orbitals (FP-LMTO) method based on the local density approximation (LDA) as the most accurate in describing the electronic structure of 
solids. A general description of FP-LMTO is presented in Chapter 2. The computational details for site-preference problem of $\mathrm{Mo}$ and $\mathrm{V}$ in $\mathrm{Fe}_{3} \mathrm{Al}$ are outlined in Chapter 3. Chapter 3 also contains the results of the calculations together with their physical interpretation.

Our second task was to calculate the thermal expansion of pure iron-aluminides and the thermal expansion with the addition of Molybdenum and Vanadium. In pure iron-aluminides the binding between the atoms has both metallic and covalent features. In covalent crystals the optical phonons have relatively high activation energy and make insignificant contribution to the thermal expansion at temperatures comparable with the Debye temperature. Thus we used FPLMTO method incorporated into the Debye model to calculate the thermal expansion of pure $\mathrm{Fe}_{3} \mathrm{Al}$ and $\mathrm{FeAl}$. The results of the calculations are presented in Chapter 4.

The presence of point defects, in our case, transition metal additives, can significantly affect the phonon spectrum of iron-aluminides and therefore, the thermal expansion. To examine this statement, we performed molecular dynamics simulation based on many-body atomistic potentials obtained purely from first-principle calculations. Then we compared the results with the thermal expansion calculated within the Debye model using the same atomistic potentials. While for pure iron-aluminides the difference between two methods becomes noticeable only at high temperatures, in the presence of transition metal additives the results strongly differ from each other indicating that the Debye model becomes invalid for these compounds. Our manybody atomistic potentials, their fitting procedure and the results of calculations are presented in Chapter 5. 


\section{References}

1. A summary of experimental studies of iron-aluminides can be found in: N.S.Stoloff, Proceedings of the International Symposium on Nickel and Iron Aluminides: Processing, Properties and Applications, edited by S.V.Deevi, P.J.Maziasz, V.K.Sikka and R.W.Cahn (ASM International, Ohio, 1997)

2. M.A.Crimp, K.Vendula, J Mater Sci, vol.78, 1986, p.193

3. C.G.McKamey, J.A.Horton, C.T.Liu, Scr Metall, vol.36, 1988, p.2947-2958

4. C.G.McKamey, J.A.Horton, C.T.Liu, J Mater Res, vol.4, 1989, p.1156-1163

5. T.B.Massalski, Ed., Binary Alloy Phase Diagrams, vol.1 \&2, American Society of Metals, 1986

6. Y.Nishino, C.Kumada, S.Asano, Scripta Materialia, vol.36, N.4, 1997, p.461-466

7. C.G.McKamey, P.J.Maziasz, J.W.Jones, J Mater Res, vol.7, N.8, 1992, p.2089-106

8. Insoo Kim, W.D.Cho, Materials Letters 40, 1999, p. 124-128 


\section{Chapter 2}

\section{Full-Potential Linear Muffin-Tin Orbital Method (FP-LMTO)}

\subsection{Basis of one-electron local potential theory}

The problem of electrons in a solid is, in principle, a many-electron problem that requires solving the Schrödinger equation for the $N$-particle wave function of all $N$-electrons in a solid, $\Psi\left(r_{1}, s_{1}, r_{2}, s_{2} \ldots \ldots, r_{n}, s_{n}\right): H \Psi\left(r_{1}, r_{2}, \ldots \ldots, r_{n}\right)=E \Psi\left(r_{1}, r_{2}, \ldots \ldots, r_{n}\right)$,

where the Hamiltonian $H$ is:

$$
H \Psi=-\sum_{i} \frac{\hbar^{2}}{2 m} \nabla_{i}^{2} \Psi-Z e^{2} \sum_{i, R} \frac{1}{\left|\vec{r}_{i}-\vec{R}\right|} \Psi+\frac{1}{2} \sum_{i \neq j} \frac{e^{2}}{\left|r_{i}-\vec{r}_{j}\right|} \Psi
$$

Here, the first term represents the sum of the kinetic energies of the individual electrons, the second defines the interaction of each of these electrons with the nuclei located at the lattice site $\mathbf{R}$, and the last term contains the repulsive Coulomb interaction energy between pairs of electrons. In macroscopic case the value of $N$ will be the order of $10^{23}$, so it is unfeasible to solve (2.1) exactly.

Historically, the first approximation for solving a many-electron problem was proposed by Hartree. His idea can be summarized as follows: instead of working with the total wave function of the system, each electron should be assigned its personal wave function and energy level. Certainly, this is only possible when the correlation effects are small in the electronic system and a motion of every electron can be approximated independently of each other. Furthermore, the interaction between the given electron and all other electrons is replaced by the action of an electrostatic field induced by the charge density averaged over the states of these 
electrons. The total wave function of the system is represented as a product of the single-electron wave functions:

$\Psi\left(\overrightarrow{r_{1}}, \overrightarrow{r_{2}}, \ldots \ldots, \overrightarrow{r_{n}}\right)=\varphi_{1} \varphi_{2} \ldots . . \varphi_{n}$

where each of the orbitals $\varphi_{i}$ satisfies the equation:

$$
H_{i} \varphi_{i}(\vec{r})=\left(-\nabla_{i}^{2}+V_{i o n}(\vec{r})+V_{e l}(\vec{r})+V_{i}(\vec{r})\right) \varphi_{i}(\vec{r})=\varepsilon_{i} \varphi_{i}(\vec{r})
$$

Here, $\nabla^{2}$ is the kinetic energy operator; $V_{\text {ion }}(\boldsymbol{r})$ is the Coulomb potential energy of an electron at the point $\boldsymbol{r}$ resulting from the interaction with the nuclei and $V_{e l}(r)$ represents the electron interaction with the total electronic charge density. The last term can be written in the following form:

$V_{e l}(\vec{r})=-e \int d r^{\prime} \rho\left(r^{\prime}\right) \frac{1}{\left|\vec{r}_{i}-\vec{R}\right|}$

where the electronic charge density $\rho\left(r^{\prime}\right)$ is defined:

$\rho(r)=-e \sum_{i}\left|\Psi_{i}(r)\right|^{2}$

One can see that $V_{e l}(r)$ includes the electron self-interaction. To compensate for this, the third term in $(2.3), V_{i}(\boldsymbol{r})$, is used:

$V_{i}(\vec{r})=-n_{i} \int 2 \frac{\varphi_{i}^{*}\left(\vec{r}^{\prime}\right) \varphi_{i}(\vec{r})}{\left|\vec{r}^{\prime}-\vec{r}\right|} d \vec{r}^{\prime}$

where $n_{i}$ is the number of the electrons in orbital $i$.

However, in the Hartree approximation the total wave function is symmetric with respect to the interchanging of electron pairs and therefore, it does not satisfy the Pauli principle. The Hartree-Fock method corrects this deficiency by writing the total wave function as the determinant: 


$$
\Psi\left(\vec{r}_{1}, \vec{r}_{2}, \ldots, \vec{r}_{N}\right)=\frac{1}{N}\left|\begin{array}{cccc}
\varphi_{1}\left(\overrightarrow{r_{1}}\right) & \varphi_{1}\left(\overrightarrow{r_{2}}\right) & \ldots & \varphi_{1}\left(\overrightarrow{r_{N}}\right) \\
\varphi_{2}\left(\overrightarrow{r_{1}}\right) & \varphi_{2}\left(\overrightarrow{r_{2}}\right) & \ldots & \varphi_{2}\left(\overrightarrow{r_{N}}\right) \\
\vdots & \vdots & \ddots & \vdots \\
\varphi_{N}\left(\overrightarrow{r_{1}}\right) & \varphi_{N}\left(\overrightarrow{r_{2}}\right) & \cdots & \varphi_{N}\left(\overrightarrow{r_{N}}\right)
\end{array}\right|
$$

When we substitute this wave function into the Schrödinger equation, and use the Ritz variational principle:

$$
\delta\left[\frac{\int \Psi^{*}(\mathrm{H}-E) \Psi d V}{\int \Psi^{*} \Psi d V}\right]=0
$$

we obtain the Hartree-Fock equation:

$$
\left[-\nabla^{2}+V_{C}(\vec{r})\right] \varphi_{i}(\vec{r})-\sum_{j} n_{j} \varphi_{j}(\vec{r}) \int 2 \frac{\varphi_{j}^{*}\left(\vec{r}^{\prime}\right) \varphi_{i}\left(\overrightarrow{r^{\prime}}\right)}{\left|\vec{r}^{\prime}-\vec{r}\right|} d \vec{r}^{\prime}=\varepsilon_{i} \varphi_{i}(\vec{r})
$$

The equation (2.9) and Hartree equation (2.3) differ only in the third term that is called the exchange term. The complexity introduced by the exchange term is considerable, since it represents an integral operator and it is nonlinear in $\varphi$. The Hartree-Fock equations (2.9) can be solved by special iteration techniques. First, the initial set of orbitals $\varphi_{i}(\mathbf{r})$ is selected and substituted into (2.9). Then, by solving the eigenvalue problem, another set of orbitals is obtained and, this process gets repeated until self-consistency is achieved.

At present, the Hatree-Fock method is the best one-electron method. It has also been most effective in calculating the electronic structure of finite many-particle systems, i.e., atoms. However, its application to macroscopic many-electron systems presents some difficulties. For the Fermi statistics of macroscopic systems, when there is a strong dependence of the exchange energy on $\vec{k}$, the one-electron energies determined by Hartree-Fock are not correct. 


\subsection{Density Functional Theory (DFT)}

\subsubsection{Formalism}

The Density Functional Theory (DFT) is based on Hohenberg-Kohn theorem [1]. This theorem states that the total energy of any system of electrons and nuclei is a functional of the position of the nuclei and the density of the electrons. Kohn and Sham [2] proposed the way this theorem can be used for self-consistent electronic structure calculations.

The standard Hamiltonian of the system can be written in the following functional form:

$$
E[\rho]=G[\rho]+\int d \vec{r} \rho(\vec{r})\left[V_{e x t}(\vec{r})+\frac{1}{2} \Phi(\vec{r})\right]
$$

where $V_{\text {ext }}(\vec{r})$ is the external field incorporating the field of the nuclei, $\Phi$ is the classical Coulomb potential for electrons, and the functional $G[\rho]$ includes the kinetic and exchangecorrelation energy of electrons [3].

$$
G[\rho]=T_{0}[\rho]+E_{x c}[\rho]
$$

Here, $T_{0}[\rho]$ is the kinetic energy of a system of non-interacting electrons with density $\rho(\vec{r})$ and the functional $E_{x c}[\rho]$ may be viewed as a definition of the exchange-correlation energy. The exact form of $E_{x c}[\rho]$ includes the Hartree-Fock exchange energy, the many body correlation corrections and a cancellation of the electrons self-interaction in $\Phi$. Since $T_{0}[\rho]$ is different from the true kinetic energy $T$, the exchange-correlation term includes the remainder of the kinetic energy not found in $T_{0}[\rho]$.

The application of the variational principle to (2.10) yields:

$$
\frac{\delta E[\rho]}{\delta \rho(\vec{r})}=\frac{\delta T_{0}[\rho]}{\delta \rho(\vec{r})}+V_{\text {ext }}(\vec{r})+\Phi(\vec{r})+\frac{\delta E_{x c}[\rho]}{\delta \rho(\vec{r})}
$$


If we rewrite this equation using the following definition of the effective potential:

$$
V(\vec{r})=V_{e x t}(\vec{r})+\Phi(\vec{r})+V_{x c}(\vec{r}),
$$

where the exchange-correlation potential $V_{x c}$ is

$$
V_{x c}(\vec{r})=\frac{\delta E_{x c}[\rho]}{\delta \rho(\vec{r})},
$$

we obtain the following equation:

$$
\frac{\delta E[\rho]}{\delta \rho(\vec{r})}=\frac{\delta T_{0}[\rho]}{\delta \rho(\vec{r})}+V(\vec{r})
$$

This equation is mathematically identical to the Schrödinger equation for non-interacting particles,

$$
\left[-\frac{1}{2} \nabla^{2}+V(\vec{r})\right] \psi_{i}(\vec{r})=\varepsilon_{i} \psi_{i}(\vec{r})
$$

with the charge density as defined in (2.5). The solution of this system of equations then leads to the energy and density of the lowest state and to all quantities that can be derived from them.

Although the DFT is applicable only to the ground state, the importance of this theory to practical applications can hardly be overestimated, since it reduces the many-electron problem to an essentially single-particle problem with the effective local potential $V(\vec{r})=V_{\text {ext }}(\vec{r})+\Phi(\vec{r})+V_{x c}(\vec{r})$. Obviously, (2.16) can only be solved self-consistently, since $V(\vec{r})$ depends on the orbitals $\varphi_{i}(\mathrm{r})$ that we are seeking.

In Eq. 2.12, all the terms but the exchange-correlation energy $E_{x c}[\rho]$ can be evaluated exactly, so the approximation for exchange-correlation potential plays a central role in applying DFT. For inhomogeneous electron gas the exact expression of $E_{x c}[\rho]$ can be expressed as the 
Coulomb interaction between the electron with its surrounding, so called an exchange-correlation hole and charge density $\rho_{x c}\left(\vec{r}, \vec{r}^{\prime}-\vec{r}\right)[4,5]$ :

$$
E_{x c}[\rho]=\frac{1}{2} \int d \vec{r} \rho(\vec{r}) \int d \vec{r}^{\prime} \frac{\rho_{x c}\left(\vec{r}, \vec{r}^{\prime}-\vec{r}\right)}{\left|\vec{r}-\vec{r}^{\prime}\right|},
$$

with charge density defined as

$$
\rho_{x c}\left(\vec{r}, \vec{r}^{\prime}-\vec{r}\right) \equiv \rho\left(\vec{r}^{\prime}\right) \int_{0}^{2} d \lambda\left[g\left(\vec{r}, \vec{r}^{\prime} ; \lambda\right)-1\right] .
$$

where $g\left(\vec{r}, \vec{r}^{\prime} ; \lambda\right)$ is the pair correlation function; $\lambda$ is the coupling constant.

The $E_{x c}[\rho]$ is independent of the actual shape of the exchange-correlation hole. It can be shown [6] by replacing the variables $\vec{R}=\vec{r}-\vec{r}^{\prime}$ that

$$
E_{x c}[\rho]=4 \pi \int d \vec{r} \rho(\vec{r}) \int \vec{R} d \vec{R} \rho_{x c}(\vec{r}, \vec{R})
$$

and depends only on the spherically averaged charge density

$$
\bar{\rho}_{x c}(r, R)=\frac{1}{4 \pi} \int d \Omega \bar{\rho}_{x c}(\vec{r}, \vec{R})
$$

This means that the Coulomb energy depends only on the distance, not on the direction. Furthermore, the hole-charge density satisfies the sum rule [6]:

$$
4 \pi \int R^{2} d R \bar{\rho}_{x c}(r, R)=-2
$$

\subsubsection{Local Density Approximation (LDA)}

In band structure calculations, usually some certain approximations for the exchangecorrelation potential $V_{x c}(\vec{r})$ are used. The simplest and most frequently used is the local density approximation (LDA), where $\rho_{x c}\left(\vec{r}, \vec{r}^{\prime}-\vec{r}\right)$ has a form similar to that for a homogenous electron 
gas, but with the density at every point of the space replaced by the local value of the charge density $\rho(\vec{r})$ :

$$
\rho_{x c}\left(\vec{r}, \vec{r}^{\prime}-\vec{r}\right)=\rho(\vec{r}) \int_{0}^{2} d \lambda\left[g_{0}\left(\left|\vec{r}-\vec{r}^{\prime}\right|, \lambda, \rho(\vec{r})\right)-1\right]
$$

where $g_{0}\left(\left|\vec{r}-\vec{r}^{\prime}\right|, \lambda, \rho(\vec{r})\right)$ is the pair correlation function of a homogenous electron system. This approximation also satisfies the sum rule (2.22), which is one of its basic advantages. By substituting (2.23) into (2.18) we obtain the local electron density approximation:

$$
E_{x c}[\rho]=\int \rho(\vec{r}) \mathcal{E}_{x c}(\rho) d \vec{r}
$$

Here, $\varepsilon_{x c}$ is the contribution of exchange and correlation to the total energy (per electron) of a homogeneous interacting electron gas with the density $\rho(\vec{r})$. This approximation is pretty significant when $\rho(\vec{r})$ varies slowly. One of the most commonly used interpolation expressions for $\varepsilon_{x c}(\rho)$ was given by Hedin and Lundqvist [7]. In the local density approximation, the effective potential (2.16) is

$$
V(\vec{r})=V_{\text {ext }}(\vec{r})+\Phi(\vec{r})+\mu_{x c}(\vec{r})
$$

where $\mu_{x c}(\vec{r})$ is the exchange-correlation part of the chemical potential of a homogeneous interacting electron gas with the local density $\rho(\vec{r})$,

$$
\mu_{x c}(\vec{r})=\frac{d \rho \varepsilon_{x c}(\rho)}{d \rho}
$$

For spin-polarized systems, the local spin density approximation[3,8] is used

$$
E_{x c}\left[\rho^{+}, \rho^{-}\right]=\int \rho(\vec{r}) \varepsilon_{x c}\left(\rho^{+}(\vec{r}), \rho^{-}(\vec{r})\right) d \vec{r}
$$

Here, $\varepsilon_{x c}\left(\rho^{+}(\vec{r}), \rho^{-}(\vec{r})\right)$ is the exchange-correlation energy per electron of a homogeneous system with the densities $\rho^{+}(\vec{r})$ and $\rho^{-}(\vec{r})$ for spins up and down, respectively. 


\subsection{Full-Potential Linear Muffin-Tin Orbital Method (FP-LMTO)}

\subsubsection{Calculation of Energy Bands in Crystals}

The computation of the electronic structure of a crystal can be divided into two parts: (i) the definition of an effective crystal potential, and (ii) the actual solution of the Schrödinger equation to find the corresponding Bloch wave functions in this potential. It was shown in previous chapter how the many-electron problem of solids can be reduced within DFT to a single-electron problem with the corresponding effective potential (2.13). In this chapter we discuss the methods of solving the Schrödinger equation (2.16).

The electronic structure calculation in a solid is strongly simplified by the crystal symmetry. This yields that the effective potential, $V(\vec{r})$ can be represented by a periodic function: $V(\vec{r}+\vec{R})=V(\vec{r})$, where $\vec{R}$ is the translation vector of the lattice that is defined as:

$$
\vec{R}=\mathrm{m}_{1} \mathbf{a}_{1}+\mathrm{m}_{2} \mathbf{a}_{2}+\mathrm{m}_{3} \mathbf{a}_{3}
$$

The vectors $\left\{\mathbf{a}_{\mathbf{i}}\right\}$ are the real-space Bravais lattice vectors that span the crystal cell and $\left\{\mathrm{m}_{\mathrm{i}}\right\}$ are integers. The Bloch's theorem states that the eigenstates $\Psi$ of the one-electron Hamiltonian with periodic effective potential can be written in the form of a plane wave times a function with the periodicity of the Bravais lattice:

$$
\Psi_{\vec{k}}(\vec{r})=\exp (i \vec{k} \cdot \vec{r}) u_{\vec{k}}(\vec{r})
$$

where $\vec{k}$ is called a wave vector and $u_{k}(r)$ is a periodic function that satisfies:

$$
u_{\vec{k}}(\vec{r}+\vec{R})=u_{\vec{k}}(\vec{r})
$$


By substituting (2.28) into the Schrödinger equation (2.16) one can obtain the dependence of the energies $\varepsilon_{i}$ on the wave vector $k$. The collection of the functions $\varepsilon_{i}(k)$ represents the band structure of a solid. This is one of the most fundamental physical characteristics of a crystal, since it describes the atomic binding and provides an explanation of electronic transport properties of solids.

In computing the electronic structure of a crystal, almost all band theory methods use the same approach. The wave function of a valence electron in a crystal, which satisfies the Bloch condition, is written in the form of a linear combination of basis functions:

$$
\Psi(\vec{r})=\sum_{i} C_{i} \varphi_{i}(\vec{r})
$$

The exact form of functions $\varphi_{i}(r)$ depends on the choice of computational method. Then, using the Ritz variational principle, the Schrödinger second-order differential equation can be reduced to an algebraic problem of eigenvalues and eigenvectors. Equation (2.30) is substituted into (2.8) and the variation is carried out with respect to the coefficient $C_{i}$ to obtain the following secular equations:

$$
\sum_{i}\left(H_{i j}-E O_{i j}\right) C_{i}=0 \quad j=1,2, \ldots, N
$$

where $N$ is the numbers of basis functions. $H_{i j}$ and $O_{i j}$ are called Hamiltonian and overlap matrix elements, respectively. They are defined as follows:

$$
\begin{aligned}
& H_{i j}=\int \varphi_{i}^{*}(\vec{r}) H \varphi_{j}(\vec{r}) d r \\
& O_{i j}=\int \varphi_{i}^{*}(\vec{r}) \varphi_{j}(\vec{r}) d r
\end{aligned}
$$

The function $E(\vec{k})$ is defined by:

$$
\operatorname{det}\left|H_{i j}-E O_{i j}\right|=0
$$


There are two kinds of limiting representations of a valence electron, that is, tightly bound and nearly free electron.

When the valence electron is well localized with respect to atomic nucleus, for instance, when the electron has $d$ or $f$ orbital quantum number, the wave function can be written in the form of a linear combination of atomic orbitals centered at a lattice site. The problem is that the wave function of a valence electron in the vicinity of a given atom contains not only the orbitals of this atom but also the tails of the orbitals of neighboring atoms, this will lead to computational difficulty in the calculation of overlapping integrals. Also, this method cannot be used in the conduction band or for diffuse high-energy states, since the basis set of atomic orbitals does not form a complete set of functions to describe these states.

For a nearly free electron, the wave function is written as an expansion in plane waves, that is, a Fourier series. This assumes that the potential energy of the electron in the periodic field of the crystal is small relative to its kinetic energy. However, in the core region of atoms, the potential is not small and the wave functions strongly oscillate. Thus, to reproduce such behavior in the wave functions by a linear combination of plane waves, it is necessary to use a large set. Therefore, although this is a comparatively simple picture in physics, it becomes exceedingly complicated in computation for some systems.

Computation methods in the different band theories differ only in the selection of the basis function $\varphi_{i}$.

\subsubsection{The FP-LMTO method used in this dissertation}

The specific technique employed in this dissertation is one of several commonly used Full-Potential Linear Muffin-Tin orbital methods, which was developed by Wills and his co- 
workers. The detailed description can be found in Ref. 9. Here, we outline the most important computational features.

\section{Notation:}

While describing the electronic structure calculations, one unavoidably is going to use an enormous number of symbols and indices. To avoid any confusion, we briefly describe the notations used in this chapter.

\section{Spherical harmonics:}

$$
\begin{aligned}
& y_{l m}(\hat{r}) \equiv i^{l} Y_{l m}(\hat{r}) \\
& C_{l m}(\hat{r}) \equiv \sqrt{\frac{4 \pi}{2 l+1}} Y_{l m}(\hat{r}) \\
& c_{l m}(\hat{r}) \equiv i^{l} C_{l m}(\hat{r})
\end{aligned}
$$

where $Y$ is a spherical harmonic.

\section{Bessel functions:}

$$
\begin{aligned}
& \mathrm{K}_{l}(\kappa, r) \equiv-\kappa^{l+1} \begin{cases}n_{l}(\kappa r)-i j_{l}(\kappa r) & \kappa^{2}<0 \\
n_{l}(\kappa r) & \kappa^{2}>0\end{cases} \\
& \mathrm{K}_{L}(\kappa, \vec{r}) \equiv \mathrm{K}_{l}(\kappa, r) y_{L}(\hat{r}) \\
& J_{l}(\kappa, r) \equiv j_{l}(\kappa r) / \kappa^{l} \\
& J_{L}(\kappa, \vec{r}) \equiv J_{l}(\kappa, r) y_{L}(\hat{r})
\end{aligned}
$$

where $L$ denotes $l m$, and $n_{l}$ and $j_{l}$ are spherical Neuman and Bessel functions, respectively. 


\section{Geometry}

FP-LMTO method is based on so called muffin-tin geometry. In this approach the crystal is divided into non-overlapping spheres surrounding atomic sites, so called muffin-tin spheres, where the density and potential vary rapidly, and the interstitial region between the spheres, where the density and potential vary slowly. The same concept was used in the early electronic structure methods such as KKR and APW. However, in FP-LMTO the division is only a computational one, and does not restrict the final shape of the density or potential. Inside the muffin tins, the basis functions, electron density, and potential are expanded in spherical waves; in the interstitial, the basis function, electron density, and potential are expanded in Fourier series.

One of the most important questions is a choice of the muffin-tin radii. Assuming all expansions are taken to convergence, the charge density and potential depend on the muffin tin radii only through the dependence of basis functions on the radii. As discussed below, basis functions have a different functional form inside the muffin tins. The choice of muffin-tin radius affects this crossover. Hence, assuming the Hamiltonian is the same inside and outside the spheres, the muffin tin radii are variational parameters and the optimum choice minimizes the total energy. If the basis is large enough, the energy is insensitive to the choice of radii. However, relativistic effects are usually taken into account only in the muffin tin spheres and in this case the Hamiltonian depends on the muffin-tin radii. Therefore, when relativistic effects are important, the radii cannot be regarded as merely variational parameters. 


\section{Potential}

Inside the muffin-tin spheres the potential is expressed as the sum of the lattice harmonics:

$$
V_{\alpha}(r)=\sum_{h} V_{\alpha, h}(r) D_{\alpha, h}(r)
$$

where the functions $D_{\alpha, h}(r)$ are the lattice harmonics invariant under point-group operations and $\alpha$ runs over nonequivalent spheres in the unit cell. In the interstitial, the potential is expressed in Fourier series:

$$
V_{i}(r)=\sum_{G} e^{i G \cdot r} V(G)
$$

The sum in Equation (2.43) is carried over the symmetry stars of the reciprocal lattice. The zero of potential is set so that the average potential in the interstitial region is zero.

Given the input potential, expressed as above, the FP-LMTO calculation proceeds by first forming a muffin-tin true potential (spherically averaged in the spheres and zero in the interstitial region), and then using this muffin-tin potential to construct basis states.

\section{Basis Set}

\section{a. Interstitial}

In the interstitial region, $I$, bases are Bloch sums of spherical Hankel or Neumann functions multiplied by the spherical harmonics:

$$
\left.\psi_{i}(\vec{k}, \vec{r})\right|_{r \in I}=\sum_{R} e^{i \vec{k} \cdot \vec{R}} \mathrm{~K}_{l_{i}}\left(\kappa_{i},\left|\vec{r}-\vec{\tau}_{i}-\vec{R}\right|\right) y_{l_{i} m_{i}}\left(D_{\tau_{i}}\left(\left|\vec{r}-\vec{\tau}_{i}-\vec{R}\right|\right)\right)
$$

The rotation $D_{\tau}$ takes the argument into a coordinate system local to each site $\tau$ and the summation is carried over all lattice vectors $R$. The function on the right hand side of (2.44) is 
sometimes called the envelope function. The basis function (2.44) is specified by the following parameters: lattice sites $\tau$ in the unit cell on which the spherical wave is based, the angular momentum parameters $l$ and $m$ of the spherical wave with respect to its parent cell, and the kinetic energy $\kappa^{2}$ of the basis in the interstitial. The angular parameters $l$ and $m$ represent the atomic states from which crystal eigenstates are derived. In our FP-LMTO method for each set of $l$ and $m$ there are several corresponding values of parameter $\kappa^{2}$. The use of "multiple $k s$ " provides a better description of the electronic wave function in the interstitial area, which is essential for calculating electronic properties of open-structure crystals and the systems with low symmetry.

There is no simple algorithm to pick a good set of interstitial kinetic energy parameters. However, the optimum set would minimize the total energy. This can be done, but it is time consuming, even for relatively simple systems.

\section{b. Muffin Tins}

In the muffin tin spheres, bases are linear combinations of spherical waves matching continuously and differentiably to the envelope function at the muffin-tin sphere. The envelope function $K$ may be expanded in a series of spherical Bessel functions about any site except it's center and the basis function on a muffin-tin sphere at $\boldsymbol{R}=0$ is given by:

$$
\begin{aligned}
\left.\psi_{i}(\vec{k}, \vec{r})\right|_{r_{\tau}=s_{\tau}} & =\sum_{R} e^{i \vec{k} \cdot \vec{R}} \sum_{L} y_{L}\left(D_{\tau} \hat{r}_{\tau}\right)\left(\mathrm{K}_{l}\left(\kappa_{i}, s_{\tau}\right) \delta(\vec{R}, 0) \delta\left(\tau, \tau_{i}\right) \delta\left(L, L_{i}\right)+J_{L}\left(\kappa, s_{\tau}\right) B_{L, L_{i}}\left(\kappa_{i}, \vec{\tau}-\vec{\tau}^{\prime}-\vec{R}\right)\right) \\
& =\sum_{L} y_{L}\left(D_{\tau} \hat{r}_{\tau}\right)\left(\mathrm{K}_{l}\left(\kappa_{i}, s_{\tau}\right) \delta\left(\tau, \tau_{i}\right) \delta\left(L, L_{i}\right)+J_{L}\left(\kappa, s_{\tau}\right) B_{L, L_{i}}\left(\kappa_{i}, \vec{\tau}-\vec{\tau}^{\prime}, \vec{k}\right)\right)
\end{aligned}
$$

where $\vec{r}_{\tau}=\vec{r}-\vec{\tau}$ and $B$ is equivalent to the KKR structure constant [10]. The unitary transformation applied to $B$ rotates components into site-local coordinates from the left and right. Equation (2.45) can be compactly expressed by defining a two-component row vector $K$ so that: 


$$
K_{l}(\kappa, r)=\left(\mathrm{K}_{l}(\kappa, r), J_{l}(\kappa, r)\right)
$$

and a two component column vector $S$ so that

$$
S_{L, L^{\prime}}\left(\kappa, \vec{\tau}-\vec{\tau}^{\prime}, \vec{k}\right)=\left(\begin{array}{c}
\delta\left(\tau, \tau^{\prime}\right) \delta\left(L, L^{\prime}\right) \\
B_{L, L^{\prime}}\left(\kappa, \vec{\tau}-\vec{\tau}^{\prime}, \vec{k}\right)
\end{array}\right)
$$

Then the value of a basis function on a muffin-tin boundary is expressed simply as:

$$
\left.\psi_{i}(\vec{k}, \vec{r})\right|_{r_{\tau}=s_{\tau}}=\sum_{L} y_{L}\left(D_{\tau} \hat{r}_{\tau}\right) K_{l}\left(\kappa_{i}, s_{\tau}\right) S_{L, L_{i}}\left(\kappa_{i}, \vec{\tau}-\vec{\tau}^{\prime}, \vec{k}\right)
$$

The radial part of a basis function inside a muffin-tin sphere is a linear combination of atomic like functions $\phi$ and their energy derivatives $\dot{\phi}[11,12]$ matching continuously and differentiably to the radial function $K$ in Equation 2.48. Collecting $\phi$ and $\dot{\phi}$ in a row vector:

$$
U(e, r) \equiv(\phi(e, r), \dot{\phi}(e, r))
$$

a matching condition may be expressed as $U(e, r) \Omega(e, \kappa)=K(\kappa, s)$ and $U^{\prime}(e, r) \Omega(e, \kappa)=K^{\prime}(\kappa, s)$, where $\Omega$ is a matrix of order two.

In this method, bases corresponding to multiple principle quantum numbers are contained within a single, fully hybridizing basis set. This is accomplished simply by using functions $\phi$ and $\dot{\phi}$ calculated with energies $\left\{e_{n l}\right\}$ corresponding to different principle quantum numbers $n$ to describe the radial dependence of a basis in the muffin tins. Thus, another parameter specifying a basis function is the set of energy parameters $\left\{e_{t l}\right\}$ that will be used to calculate the radial basis functions $\phi_{t l}$ and $\dot{\phi}_{t l}$ used to express the basis function in muffin-tins of each symmetry type. A basis function in a muffin tin sphere is therefore:

$$
\left.\psi_{i}(\vec{k}, \vec{r})\right|_{r_{\tau}<s_{\tau}}=\sum_{L}^{l \leq l_{m}} U_{t L}\left(e_{i}, D_{\tau} \vec{r}_{\tau}\right) \Omega_{t l}\left(e_{i}, \kappa_{\tau}\right) S_{L, L_{i}}\left(\kappa_{i}, \vec{\tau}-\vec{\tau}^{\prime}, \vec{k}\right)
$$


where $e_{i}$ means "use the energy parameter $e_{n l}$ corresponding to the principal quantum number specified for basis i" and:

$$
U_{t L}\left(e_{i}, r\right) \equiv y_{L}(\hat{r}) U_{t L}(e, r)
$$

In principle, and as programmed, each $(\tau l \kappa)$ basis can use its own unique energy set. It is more customary to use a common energy set for a set of basis states giving rise to bands of similar energy within the scope of a particular calculation.

A parameter introduced in (2.50) is the angular momentum cutoff $l_{m}$. In most cases, a converged total energy is achieved with values $l_{m} \sim 4-6$.

\section{Calculation procedure}

The Bloch sums (2.45) are evaluated in the spheres using calculated structure functions [16], and recalculated at each iteration as the energy parameters are reset. In the interstitial region the basis states (2.44) are determined by expanding the sum of Neumann functions, each with an arbitrary, smooth extension into the muffin-tin spheres in a Fourier series. The construction of the Hamiltonian matrix elements (2.32) is carried out then either by analytic expressions or simple radial integrals in the spheres and by convolution with a step function in the interstitial region, with care taken to include the necessary number of Fourier components in the step function. The overlap matrix is constructed in a similar manner and the diagonalization of the Hamiltonian follows standard techniques. It should be pointed out that our energy windows are allowed to hybridize; that is, matrix elements connecting basis functions associated with different sets of energy parameters are included in the construction of the Hamiltonian and overlap matrix elements. 
The determination of the Fermi energy level is straightforward, and the electron density is constructed into a form analogous to the expression for the potential. Core charge that leaks from the muffin-tin spheres is added to the charge density in the interstitial and other muffin-tin spheres by Fourier synthesis of the tails which extend beyond the sphere edge.

The computation of the potential, given the density, consists of finding the electrostatic potential and the exchange-correlation potential. The latter potential is written in the form proposed by Ceperly and Alder [17] as parametrized by Perdew and Zunger [18]. The electrostatic potential is found using the method of Weinert [19]. The exchange and correlation potential is found in the interstitial region by Fourier transforming the charge density from reciprocal to real space and then back transforming the potential. The exchange and correlation potential is found in the muffin-tin spheres using a simple numerical angular integration scheme. The input and output potentials are then mixed using Broyden's mixing scheme [20], which was found to give quick convergence to self-consistency.

The total energy,

$$
E=T+E_{H}+E_{x c}=\sum_{i} \varepsilon_{i}-\int d r \rho(r) V_{e f f}(r)+E_{H}+E_{x c} \text { (2.52) }
$$

is calculated using the cancellation of the $Z / r$ terms [21] between the kinetic energy $T$ and the electrostatic (Hartree) energy $E_{H}$, and the input value for the one-electron potential, $V_{\text {eff }}(r)$, is used in (2.53) to obtain quick convergence of the total energy . $E_{x c}$ is the net exchangecorrelation energy and is calculated in a manner similar to the construction of the exchangecorrelation potential. Cohesive energies are obtained using a fully relativistic calculation of the associated atomic energies, using numerical procedures, which are similar to those of the FPLMTO calculation as possible. 


\section{References}

1. P. Hohenberg and W. Kohn, Inhomogeneous electron gas. Phys. Rev. B, 136, 864 (1964)

2. W. Kohn and L. J. Sham, Self-consistent equations including exchange and correlation effects. Phys. Rev. A, 140, 1133 (1956)

3. L. J. Sham and W. Kohn, One-particle properties of an inhomogeneous interacting electron gas. Phys. Rev., 145, 561 (1966)

4. J. P. Perdew and D. C. Langreth, The exchange-correlation energy of a metallic surface. Solid State Com., 17, 1425 (1975)

5. O. Gunnarsson and B. I. Lundqvist, Exchange and correlation in atoms, molecules and solids by the spin-density-functinal formalism. Phys. Rev. B. 13, 4274 (1976)

6. O. Gunnarsson and R. O. Jones, Density functional calculations for atoms, molecules and clusters. Phys. Scr., 21, 391 (1980)

7. L. Hedin and B. I. Lundqvist, Explicit local exchange-correlation potentials. J. Phys. C, 4, 2064 (1971)

8. U. Barth and L. Hendin, A local exchange-correlation potential for the spin-polarized case. J. Phys. C, 5, 1629 (1972)

9. J. M. Wills, O. Eriksson, M. Alouani, and D. L. Price, Full-Potential LMTO total energy and force calculation, Electronic Structure and Physics Properties of Solids, H. Dreysse(Ed.), Springer-Verlag, Berlin, 2000, pp 148-167.

10. J. Korringa, Physica 13, 392 (1947); W. Kohn and N. Rostoker, Phys. Rev., 94, 1111 (1954)

11. O. K. Andersen, Phys. Rev. B 12, 3060 (1988) 
12. H. L. Skriver, The LMTO method (Springer-Verlag, Belin, 1984)

13. Byoung-Ho-Cheong, C. H. Park and K. J. Chang, Phys. Rev. B 51, 10610 (1995)

14. Gun-Do-Lee, M. H. Lee, and Jisoon-Ihm, Phys. Rev. B 52, 1459 (1995)

15. S. Pöykkö, M. J. Puska, T. Korhonen, and R. M. Nieminen, Mat. Sc. Eng. B 43 1, (1997)

16. F.S.Ham and B.Segall, Phys. Rev. vol.124, 1786 (1961)

17. D.M.Ceperly and B.J.Alder, Phys.Rev.Lett, vol.45, 566 (1980)

18. J.P.Perdew and A.Zunger, Phys.Rev. B, vol.23, 5048 (1981)

19. M.Weinert, J.Math.Phys. vol.22, 2433(1981)

20. G.P.Srivastava, J.Phys. A vol.17, L317 (1984)

21. M.Weinert, E.Wimmer and A.J.Freeman, Phys.Rev. B, vol.26, 4571(1982) 


\section{Chapter 3 Site preference of Mo and $\mathrm{V}$ additives in $\mathrm{Fe}_{3} \mathrm{Al}$}

\subsection{Introduction}

The transition metal additives, particularly Mo and V, significantly modify mechanical and thermal properties of $\mathrm{Fe} 3 \mathrm{Al}$. In particular, their substitution leads to a remarkable stabilization of $\mathrm{Fe} 3 \mathrm{Al} \mathrm{DO} 3$ phase and therefore, improves the strength of this material [1]. The phase stabilization induced by additives is frequently related to their determined preference for a particular site in the atomic lattice. The goal of our research is to examine this statement.

Fe3 Al exists in the ordered DO3 structure up to the temperature 550C [2]. There are four basis atoms in the unit cell and they are sitting at the body-centered cubic lattice sites. The sixteen-atom supercell of a DO3 structure is shown in Figure 3.1. The symmetry divides the atomic sites of the lattice into the three types. Two of these types are the iron sites, namely FeI and FeII, and the third type includes all the Aluminum atoms. The FeII sites have eight iron atoms as nearest neighbors. They are shown as the red spheres in Figure 3.1. The FeII sites are surrounded by 4 iron and 4 aluminum atoms and represented by the green spheres in Figure 3.1. The Aluminum atoms, shown as the blue spheres, have 8 neighboring atoms of the FeI type. The sixteen-atom supercell of a DO3 structure has a cubic symmetry. 
Figure 3.1 $\mathrm{DO}_{3}$ structure of $\mathrm{Fe}_{3} \mathrm{Al}$

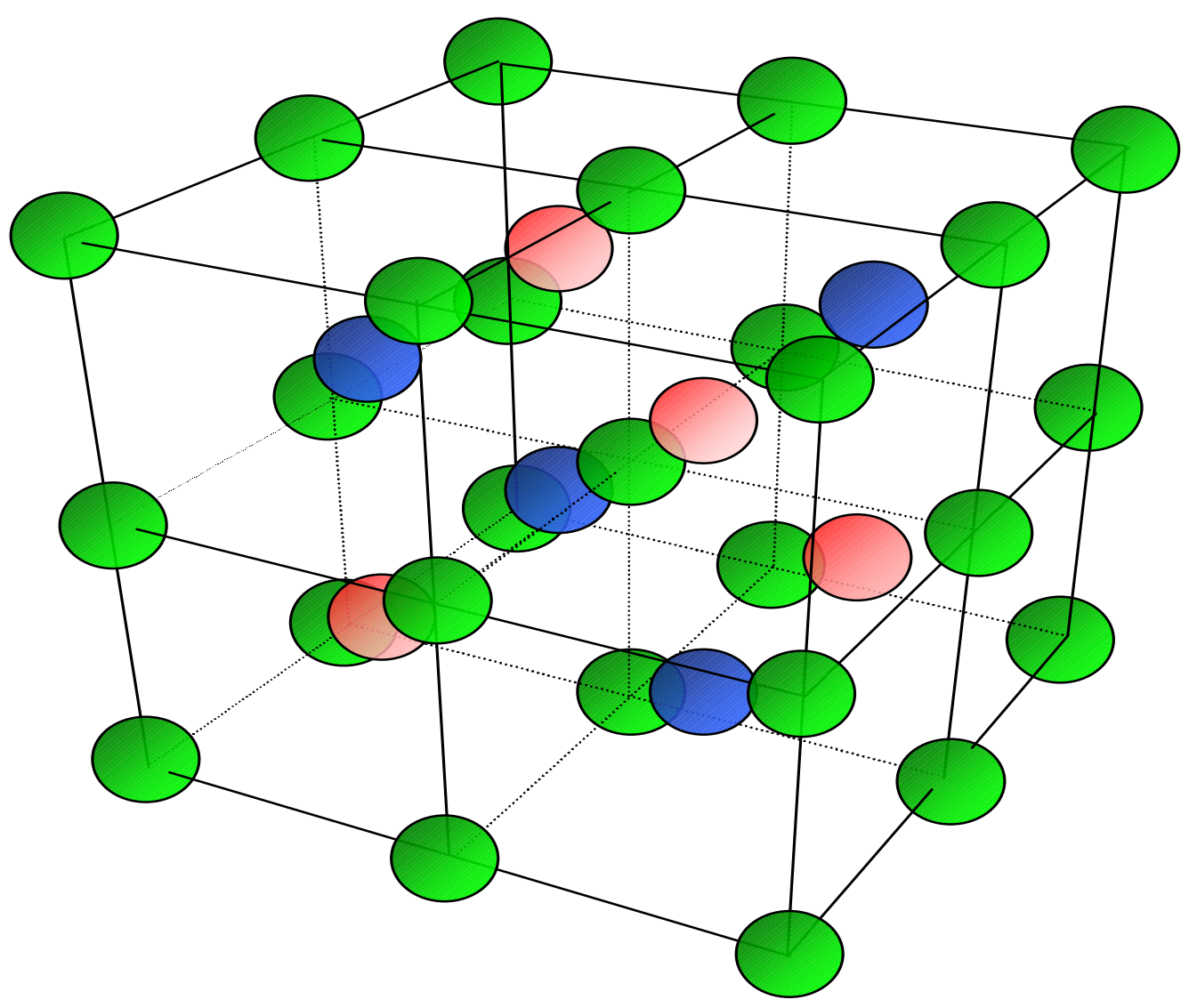

FeI

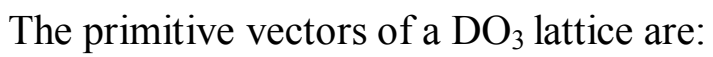

- $\quad \mathbf{A}_{1}=\mathrm{a} \mathbf{Y}+\mathrm{a} \mathbf{Z}$

- $\mathbf{A}_{2}=\mathrm{a} \mathbf{X}+\mathrm{a} \mathbf{Z}$

- $\mathbf{A}_{3}=\mathrm{a} \mathbf{X}+\mathrm{a} \mathbf{Y}$

The basis vector are given by:

- $\mathbf{B}_{1}=0(\mathrm{Al})$

- $\mathbf{B}_{2}=-1 / 2 \mathbf{A}_{1}+1 / 2 \mathbf{A}_{2}+1 / 2 \mathbf{A}_{3}=\mathrm{a} \mathbf{X}(\mathrm{FeI})$

- $\mathbf{B}_{3}=+1 / 4 \mathbf{A}_{1}+1 / 4 \mathbf{A}_{2}+1 / 4 \mathbf{A}_{3}=+1 / 2$ a $\mathbf{X}+1 / 2$ a $\mathbf{Y}+1 / 2$ a $\mathbf{Z}$ (FeII)

- $\mathbf{B}_{4}=-1 / 4 \mathbf{A}_{1}-1 / 4 \mathbf{A}_{2}-1 / 4 \mathbf{A}_{3}=-1 / 2$ a X - $1 / 2$ a $\mathbf{Y}-1 / 2$ a $\mathbf{Z}$ (FeII) 
Although the experimental data on the site preference of the transition metal additives in $\mathrm{Fe}_{3} \mathrm{Al}$ is still incomplete [3], there was an attempt to solve this problem computationally. Reddy et al. studied nature and strength of magnetic interaction of $3 d$ transition metal impurities in $\mathrm{Fe}_{3} \mathrm{Al}$ using Linear Combination of Atomic-Orbitals Molecular-Orbital (LCAO-MO) method [4]. They found that the type of the magnetic coupling of an impurity at different iron sites and siteselection of $3 d$ elements in $\mathrm{Fe}_{3} \mathrm{Al}$ is correlated with its position in the Periodic Table. Thus, impurities to the left of Fe couple antiferromagnetically to Fe while the impurities to the right of Fe couple ferromagnetically. These computational results provide a convenient way of controlling the magnetic properties of $\mathrm{Fe}_{3} \mathrm{Al}$ and can be used in deducing experimentally the location of the impurities in this compound. However, the magnetic cluster model can hardly be applied to resolve the site-preference problem in realistic iron-aluminides. The selection of a particular lattice site by an impurity happens during the alloy formation process at the temperature much higher than the Curie temperature of these alloys. Therefore, individual magnetic moments of the atoms are thermally disordered in the compound and can be averaged to zero. The chance that the distribution of the atoms between the lattice sites will change after the solidification is negligibly small, since the diffusion in the metallic systems is extremely slow.

The formation of pseudo-binary compounds $\left(\mathrm{Fe}_{1-x} M_{x}\right)_{3} \mathrm{Al}$, where $M$ stands for transition metal additive and $x$ is used for its concentration, is a complicated process. It requires repeated melting followed by the ordering heat-treatment [5]. The simulation of such a nontrivial physical process is impossible without making few approximations. One of the simplest and the most appropriate approximations is a canonical formalism. It is based on the assumption that the number of particles in the system and their temperature remain constant during the alloy formation [6]. 
Then, the process of the additive site selection can be associated with the creation of a point defect, namely impurity, in a perfect $\mathrm{Fe}_{3} \mathrm{Al}$ compound. The formation energy of a point defect can be written as:

$$
E^{f}=T E_{\text {defect }}+\mu_{\text {defect }}-T E_{\text {perfect }}
$$

where $T E_{\text {defect }}$ is the total energy of a supercell with a defect; $T E_{\text {perfect }}$ is the total energy of a corresponding perfect supecell; $\mu_{\text {defect }}$ is the change in chemical potential due to substituting original atom by the impurity. Thus, the sites associated with the smallest value of the defect formation energy have a bigger statistical probability to be occupied by the additive.

While the total energy of a supercell in (3.1) can be found straightforward from the firstprinciples calculations, the chemical potential there is determined with some uncertainty. Its value depends on the specific conditions of the alloy formation such as pressure, temperature gradient, exact composition of the alloy etc. Hence, it is extremely important which particular model is chosen for chemical potential calculation. A worthy to note approach of finding the change in chemical potential during the defect formation was proposed by V.Scott and M.Fahnle $[7,8]$. They considered the canonical ensemble of atoms assuming that in the ground state all the atoms occupy the appropriate lattice sites. Then, after taking into account all possible point defects in the crystal and minimizing the thermodynamical potential with respect to their number, one can derive the concentrations of the corresponding point defects at a given temperature. The main idea behind this approach is that the crystal exists at a perfect stochiometry and thus, the atoms substituted by the additive are most likely to create other point defects. While this approximation is valid for semiconductors, it can be hardly applied to intermetallic compounds and particularly to $\mathrm{Fe}_{3} \mathrm{Al}$. This intermetallic tends to have a structural disorder [9] and is very likely to possess various macroscopic defects such as grain-boundaries, 
precipitates, etc. Alternatively we assume that the atoms substituted by the additive are forming the precipitates and we define the chemical potential of an element as the total energy per atom being in a bulk. Then, the change in chemical potential due to the defect formation can be written as follows:

$$
\mu_{\text {defect }}=\mu_{\text {original }}-\mu_{\text {substituted }}
$$

where $\mu_{\text {original }}$ is the chemical potential of the original atom and $\mu_{\text {substituted }}$ is the chemical potential of the impurity.

\subsection{Methodology}

The calculation of the defect formation energies was carried out using FP-LMTO method that is described in details in Chapter 2. Here we want to outline the main advantages provided by FP-LMTO for solving the site-preference problem in $\mathrm{Fe}_{3} \mathrm{Al}$ compound with the Mo and $\mathrm{V}$ additives.

Our FP-LMTO method of solving the bulk density functional problem has a full potential both in the muffin-tin and interstitial regions. In addition, the muffin-tin orbitals are not constrained to have zero kinetic energy in the interstitial. These two features of FP-LMTO method are essential in accurately calculating within the LDA approximation, the total-energy and electronic structure in low-symmetry crystal systems. Multiple kappas provide basis enrichment that allows us to capture the behavior of $3 d$ and $4 d$ electrons. Another advanced feature of our FP-LMTO method is its use of multiple "energy windows" by which we determine the energy range for linearized electronic structure calculation. For example, $4 p$ electrons of Mo atom are sitting too close to Fermi energy surface to treat them as the core states. Therefore, we 
consider them as the valence electrons and assign them to a separate energy window while using different values of kappas.

To make our calculations more consistent we have chosen the same set of the basis functions for the structures with the same type of additive. Thus, for $\mathrm{V}$ substituted in $\mathrm{Fe}_{3} \mathrm{Al}$ the basis functions are $4 s, 4 p, 3 d$ and for Mo additive these are $5 s, 5 p, 4 d$. In present calculations we used 5 different kappas. The computational parameters for $\mathrm{Fe}_{3} \mathrm{Al}$ with the Mo and $\mathrm{V}$ additives can be found in Appendix, where we included the input file for our FP-LMTO program for the purpose of demonstration.

One of the major sources of systematic errors in FP-LMTO method is due to the limited sampling of k-points during the energy eigenvalues integration over the Brillouin zone. To minimize this error, we used the same set of k-points for each structure. Since the computational time is increasing proportionally with the number of the k-points, we found $2 \mathrm{k}$-points in the irreducible wedge to be an appropriate choice for 32-atom supercell.

We use total-energy supercell calculations to give the relative energies for site selection of Mo and $\mathrm{V}$ additives in $\mathrm{Fe}_{3} \mathrm{Al}$. The supercell model describes the periodicity of a crystal structure through the boundary conditions. When considering the low concentration of additives in the alloy, one has to carry the calculations for the supercell of a sufficiently large size. Thus, by taking a supercell consisting of 32 atoms and substituting one transition metal at arbitrary site, we simulate

the site-selection process for 3.125\% alloy. However, in this supercell model no account has been taken of random disorder that is always present in intermetallic alloys. To include it into the model, one has to average the site-selection energy over all possible supercell arrangements and this rapidly becomes impractical. The averaging has to be carried over the 
supercells with the non-equivalent symmetry and therefore, one has to consider at least two impurities per computational supercell. Thus, to study the effect of the alloying randomness on the site-selection in $\mathrm{Fe}_{3} \mathrm{Al}$ alloy with $3.125 \%$ of transition metal additive, it is necessary to take 64-atom supercell and carry the calculations 736 times. Since our aim is to use moderate amount of computer time, we rejected the idea of performing the supercell ensemble average.

In most cases the transition metal additives are used to compensate for the decrease of the iron concentration in iron-aluminides. However, the previous first-principles calculations showed that the Fe- $\mathrm{Al}$ antisite defect has a close to zero formation energy [9]. At the same time X-ray diffraction of $\mathrm{Fe}_{3} \mathrm{Al}$ confirmed that there is an antisite disorder present in this alloy [10]. Therefore, despite the most common composition of the pseudo-binary alloy, the occupation of Al sites by the additives cannot be excluded from consideration.

Hence, we considered a 32-atom supercell. The atom of the additive, Mo and later V, was placed at the one of three non-equivalent sites, namely FeI, FeII and Al, and after that all the atoms in the supercell were relaxed within the prescribed symmetry. The formation energies of the defects were calculated from (3.1). In order to take maximum advantage of the supercell symmetry and therefore reduce the computational cost, the origin of the lattice vectors was chosen to be at the site of the defect. To avoid the systematic errors, the total energy of the corresponding perfect supercell in Eq.3.1 has been recalculated for different origins. 


\subsection{Results and Discussion}

The calculated formation energies of the defects are presented in Table 3.1. The relaxation energy and change in the bond-length are defined with respect to the perfect $\mathrm{Fe}_{3} \mathrm{Al} 32$ atom supercell. Site-selection energy is defined with respect to the largest formation energy for a given defect. For both transition metal additives it is FeI site.

All the relaxation energies are found to be much higher than the typical relaxation energies in the metallic systems. The explanation of such an effect can be found in the nature of the interatomic bonding of $\mathrm{Fe}_{3} \mathrm{Al}$. There is a strong hybridization between the iron d-electrons and the p-electrons of the aluminum that gives the bonding a pronounced directional character. The Fermi energy level lies in the Fe-Al bonding state region shown in the Figure 3.2. Thus, the bonding strength between the atoms is very sensitive to intrinsic defects that in our case are represented by the Mo and V impurities.

Table 3.1 Effective formation energies of various defects in $\mathrm{Fe}_{3} \mathrm{Al}$.

\begin{tabular}{|c|c|c|c|}
\hline System & $\begin{array}{c}\text { Relaxation energy, } \\
\mathrm{eV} / \text { cell }\end{array}$ & $\begin{array}{c}\text { Change in the } \\
\text { bond length }\end{array}$ & $\begin{array}{c}\text { Site-selection energy, } \\
\text { K/atom }\end{array}$ \\
\hline Mo-FeI & 0.10 & $0.5 \%$ & 0.0 \\
\hline Mo-FeII & 0.41 & $2.9 \%$ & 25 \\
\hline Mo-Al & 0.077 & $0.1 \%$ & 0.0 \\
\hline V-FeI & No relaxation & $0.0 \%$ & 2018 \\
\hline V-FeII & 0.02 & $0.4 \%$ & 1231 \\
\hline V-Al & 0.025 & $-0.8 \%$ & \\
\hline
\end{tabular}


Figure 3.2 Electronic band structure of $\mathrm{Fe}_{3} \mathrm{Al}$ obtained by FP-LMTO method. There are 13 bands under Fermi energy level that correspond to $\mathrm{Al} 3 s^{2} 3 p^{1}$ and $\mathrm{Fe} 3 d^{6} 4 s^{2}$ valence electrons. The upper bands intersect the Fermi energy level indicating the metallic character of this material.

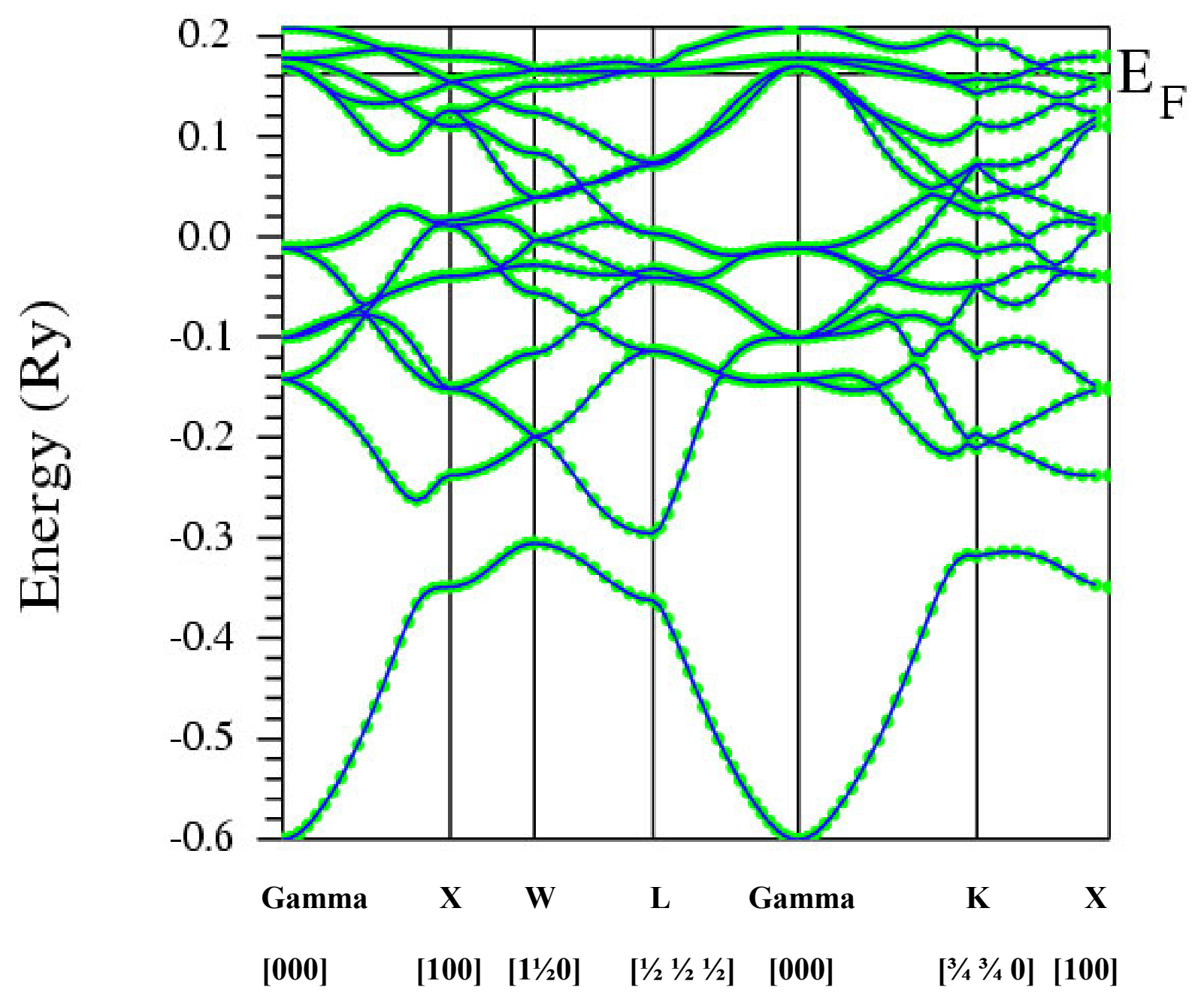


As shown in Table 3.1 the relaxation energies for Mo substituted in $\mathrm{Fe}_{3} \mathrm{Al}$ are significantly higher than their $\mathrm{V}$ counterparts. The reason for that can be found in the fact that $\mathrm{Fe}_{3} \mathrm{Al}$ gets stabilized at the lattice parameter not much larger than that of pure iron, while the atomic radius of Mo is much bigger than the radius of iron atom. Thus, Mo substitution at any site of $\mathrm{Fe}_{3} \mathrm{Al}$ causes the distortion of the lattice and the increase of the equilibrium lattice constant. The lattice distortion is particularly strong when Mo is substituted at FeII site. Here, the lattice relaxation reaches $0.41 \mathrm{eV}$ and the second neighbor distance along with the first one drastically changes. The total energy of supercell with this particular arrangement gets strongly reduced during the lattice relaxation, remains slightly larger than the total energy of a supercell with Mo atom placed at FeI site. The site-selection energy of Mo between FeI and FeII sites is only $25 \mathrm{~K}$ /atom. Taking into the account various approximations presented in our calculations, we can conclude that at non-zero temperatures Mo atoms might be distributed evenly between FeI and FeII sites. However, the site-selection energy of Mo between iron and aluminum sites is much larger, it is $763 \mathrm{~K} /$ atom. Thus, Al sites are less likely to be occupied by Mo additives.

Substitution of $\mathrm{V}$ element in $\mathrm{Fe}_{3} \mathrm{Al}$ leads to a moderate relaxation of the atomic lattice and in particular when it substituted at FeI site, no relaxation is observed. The total energy of supercell with V atom placed at FeI site is smaller than the supercell energy with V substituted at FeII site. The site-selection energy of $\mathrm{V}$ between FeI and FeII sites is relatively large, 2018 $\mathrm{K} /$ atom. This result is in a good agreement with the calculations of Reddy et al [3], although their calculations were performed for a different model and using another computational technique. Their calculated site-selection energy between FeI and FeII sites is $1870 \mathrm{~K} /$ atom. Our computational results indicate that FeI site is energetically more favorable for $\mathrm{V}$ than FeII site. However, in our calculations we included the possibility of Al sites to be occupied by the 
transition metal additives. Thus, we found that the site-selection energy between FeI and Al sites is almost half of one obtained for the two different iron sites. It is $1231 \mathrm{~K} /$ atom.

The experimental results on the site-selection of $\mathrm{V}$ in $\mathrm{Fe}_{3} \mathrm{Al}$ are quite contradictory. Nishino et al used X-ray diffraction technique to study the dependence of lattice parameter on $\mathrm{V}$ concentration $x$, in $\left(\mathrm{Fe}_{1-x} V_{x}\right)_{3} \mathrm{Al}$ compound.[1]. They found that the lattice constant decreases with the growing $x$ and reaches its minimum at about $33.3 \%$ of concentration. This result was regarded as an experimental evidence for the FeI site selection of $\mathrm{V}$ at least up to this composition range. Another research group reported the results of X-ray diffraction measurements on $\mathrm{Fe}_{2} \mathrm{VAl}$ crystals [11]. They observed appreciable antisite disorder in all of their samples. Our computational results speak in favor of the first X-ray diffraction measurements, since the obtained site-selection energy between FeI and FeII sites is relatively large. However, the small site-selection energy between $\mathrm{FeI}$ and $\mathrm{Al}$ sites is comparable to temperatures involved in the metallurgical synthesis of this iron-aluminide. Thus, we can conclude that the actual site occupation of $\mathrm{V}$ may depend significantly on the details of alloy formation. 


\section{References}

1. Y.Nishino, S.Asano, T.Ogawa, Material Science and Engineering, A234-236, (1997) p. $271-274$

2. T.B.Massalski, Ed., Binary Alloy Phase Diagrams, vol.1 \&2, American Society of Metals, 1986

3. Y.Nishino, C.Kumada, S.Asano, Scripta Materialia, vol.36, N.4, p.461-466, 1997

4. B.V.Reddy, D.H.Sastry, S.C.Deevi, S.N.Khanna, Phys.Rev. B, vol.64, 224419, 2001

5. In fact there are many different techniques of iron-aluminides synthesis. A good survey of them can be found in N.S.Stoloff, Proceedings of the International Symposium on Nickel and Iron Aluminides: Processing, Properties and Applications, edited by S.V.Deevi, P.J.Maziasz, V.K.Sikka and R.W.Cahn (ASM International, Ohio, 1997)

6. L.D.Landau, E.M.Lifshitz, Statistical Physics, Part I, "Nauka”, Moscow 1976

7. J.Mayer, C.Elsasser, M.Fahnle, Phys. Status Solidi B 191283 (1995)

8. V.Scott, M.Fahnle Phys. Status Solidi B 204617 (1997)

9. L.S.Muratov, B.R.Cooper, J.M.Wills, Mat.Res.Soc.Symp. Proc. Vol.538, 1999 Material Research Society

10. A.J.Bradley, A.H.Jay, Proc.R.Soc.London, Ser. A 136, 210 (1932)

11. Ye Feng, J.Y.Rhee, T.A. Wiener, D.W.Lynch, B.E.Hubbard, A.J.Sievers, D.L.Schlagel, T.A.Lograsso, L.L.Miller, Phys.Rev. B, vol63, 165109 (2001) 


\section{Appendix: The input data file for our FP-LMTO Program.}

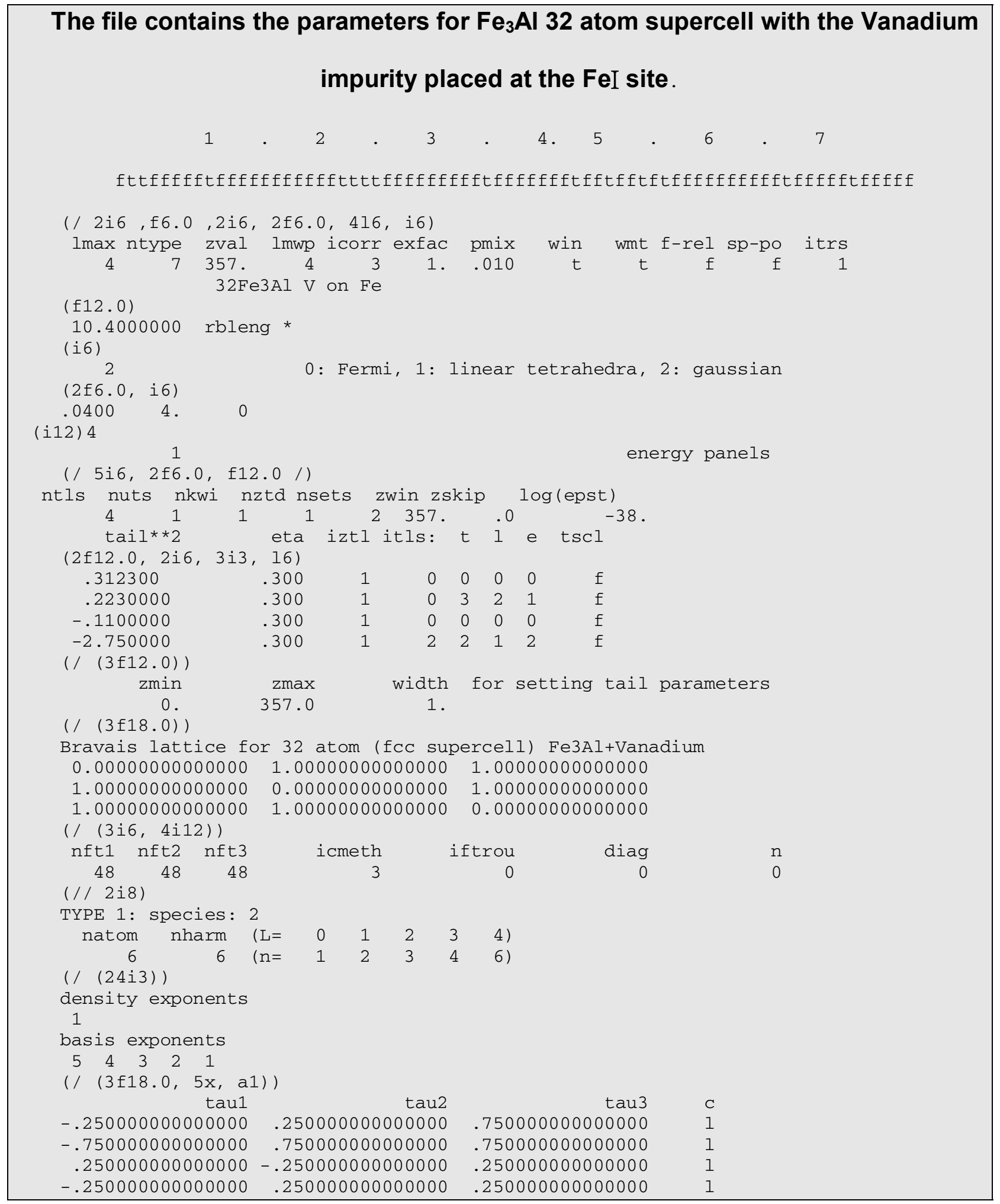




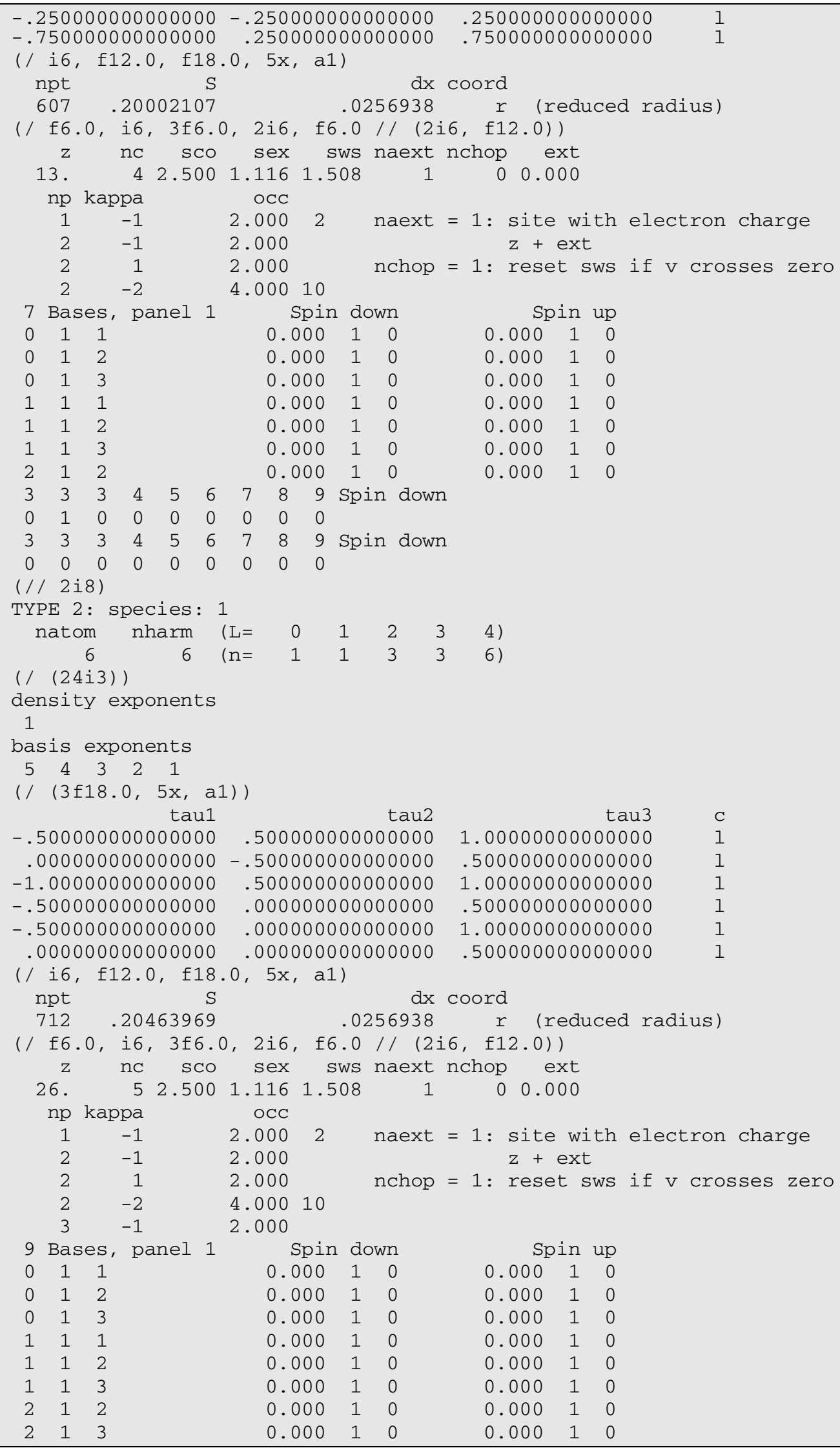




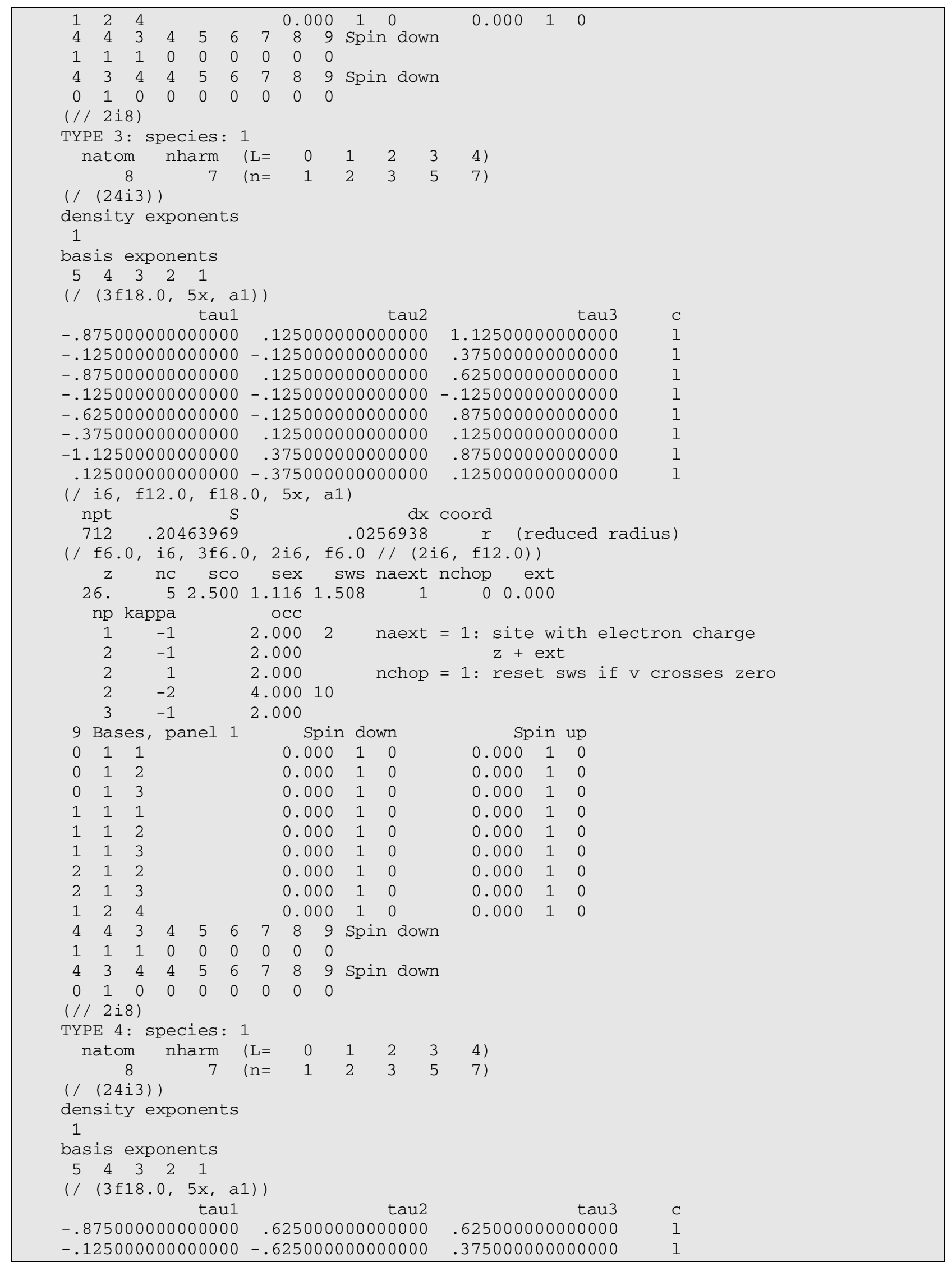




$\begin{array}{lrrr}-.375000000000000 & .125000000000000 & .625000000000000 & 1 \\ -.625000000000000 & -.125000000000000 & .375000000000000 & 1 \\ -.375000000000000 & -.375000000000000 & .625000000000000 & 1 \\ -.625000000000000 & .375000000000000 & .375000000000000 & 1 \\ -.625000000000000 & .375000000000000 & .875000000000000 & 1 \\ -.375000000000000 & -.375000000000000 & .125000000000000 & 1 \\ (/ \mathrm{i} 6, \mathrm{f} 12.0, \mathrm{f} 18.0,5 \mathrm{x}, \mathrm{a} 1) & & \end{array}$

(/ i6, f12.0, f18.0, 5x, a1)

npt $\mathrm{S} \quad \mathrm{dx}$ coord

$712.20463969 \quad .0256938 \quad r \quad$ (reduced radius)

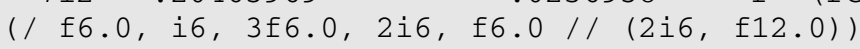

$z$ nc sco sex sws naext nchop ext

26. $52.5001 .116 \quad 1.508 \quad 1 \quad 0 \quad 0.000$

np kappa $\mathrm{OCC}$

2.0002 naext $=1$ : site with electron charge

$2.000 \quad z+$ ext

2.000 nchop $=1$ : reset sws if $\mathrm{V}$ crosses zero

4.00010

2.000

9 Bases, panel $1 \quad$ Spin down

$\begin{array}{llllll}0 & 1 & 1 & 0.000 & 1 & 0\end{array}$

$\begin{array}{llllll}0 & 1 & 2 & 0.000 & 1 & 0\end{array}$

$\begin{array}{llllll}0 & 1 & 3 & 0.000 & 1 & 0\end{array}$

$\begin{array}{llllll}1 & 1 & 1 & 0.000 & 1 & 0\end{array}$

$\begin{array}{llllll}1 & 1 & 2 & 0.000 & 1 & 0\end{array}$

$\begin{array}{lll}1 & 1 & 3\end{array}$

$\begin{array}{lll}2 & 1 & 2\end{array}$

$\begin{array}{lll}2 & 1 & 3\end{array}$

$\begin{array}{lll}1 & 2 & 4\end{array}$

$\begin{array}{llllllllll}4 & 4 & 3 & 4 & 5 & 6 & 7 & 8 & 9 & \text { Spin down }\end{array}$

$\begin{array}{lllllllll}1 & 1 & 1 & 0 & 0 & 0 & 0 & 0 & 0\end{array}$

$\begin{array}{llllllllll}4 & 3 & 4 & 4 & 5 & 6 & 7 & 8 & 9 & \text { Spin down }\end{array}$

$\begin{array}{lllllllll}0 & 1 & 0 & 0 & 0 & 0 & 0 & 0 & 0\end{array}$

(// 2i8)

TYPE 5: species: 2

natom nharm $\left(\mathrm{L}=\quad \begin{array}{lllll}0 & 1 & 2 & 3 & 4)\end{array}\right.$

(/ $(24 i 3))$

0.000110

0.000110

0.000110

0.00010

0.000110

0.000110

$0.000 \quad 1 \quad 0$

0.000110

$0.000 \quad 1 \quad 0$

0.000110

0.000110

0.00010

density exponents

1

basis exponents

$\begin{array}{lllll}5 & 4 & 3 & 2 & 1\end{array}$

(/ $(3 f 18.0,5 x, a 1))$

$\begin{array}{rrrr}\text { tau1 } & \text { tau2 } & \text { tau3 } & \mathrm{C} \\ -.750000000000000 & .250000000000000 & 1.25000000000000 & 1 \\ -.250000000000000 & -.250000000000000 & .750000000000000 & 1\end{array}$

(/ i6, f12.0, f18.0, 5x, a1)

npt $\quad \mathrm{S}$ dx coord

$607.20002107 \quad .0256938 \quad r \quad$ (reduced radius)

(/ f6.0, i6, 3f6.0, 2i6, f6.0// (2i6, f12.0))

$z$ nc sco sex sws naext nchop ext

13. $42.5001 .116 \quad 1.508 \quad 1 \quad 0 \quad 0.000$

np kappa

$1 \quad-1$

$\mathrm{OCC}$

2.000

$\begin{array}{rrr}2 & -1 & 2.000 \\ 2 & 1 & 2.000\end{array}$

2

naext

(1)

0.000

$2 \quad-2 \quad 4.00010$

7 Bases, panel 1

$\begin{array}{lll}0 & 1 & 1\end{array}$

$\begin{array}{lll}0 & 1 & 2\end{array}$

Spin down

nchop $=1$ : reset sws if $\mathrm{v}$ crosses zero

$\begin{array}{lll}0 & 1 & 3\end{array}$

$\begin{array}{lll}1 & 1 & 1\end{array}$

$\begin{array}{lll}1 & 1 & 2\end{array}$

$\begin{array}{lll}1 & 1 & 3\end{array}$

0.00010

0.00010

0.00010

0.00010

0.000110

0.00010

$\begin{array}{lll}\text { Spin } & \text { up } \\ 0.000 & 1 & 0 \\ 0.000 & 1 & 0 \\ 0.000 & 1 & 0 \\ 0.000 & 1 & 0 \\ 0.000 & 1 & 0 \\ 0.000 & 1 & 0\end{array}$




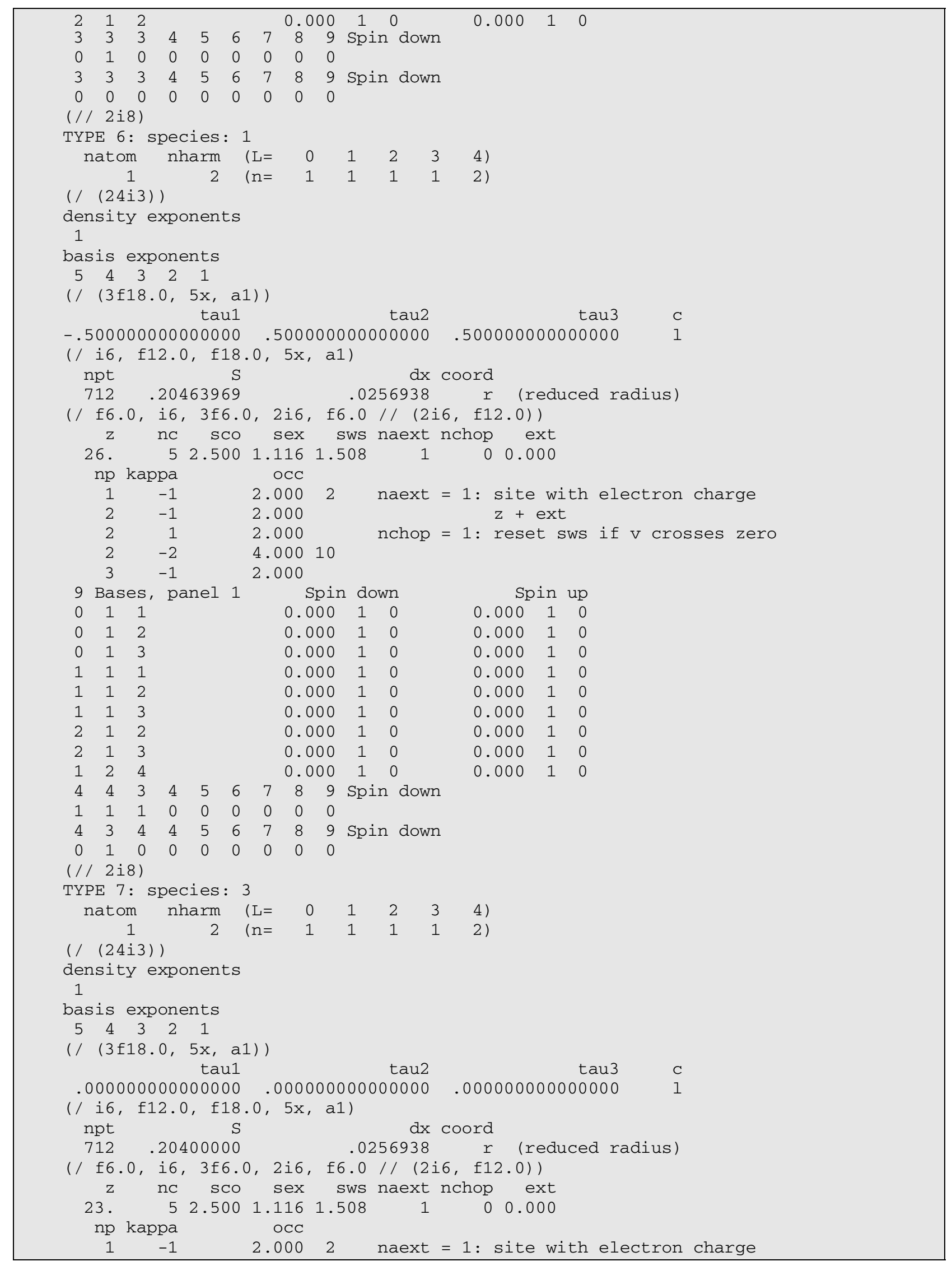




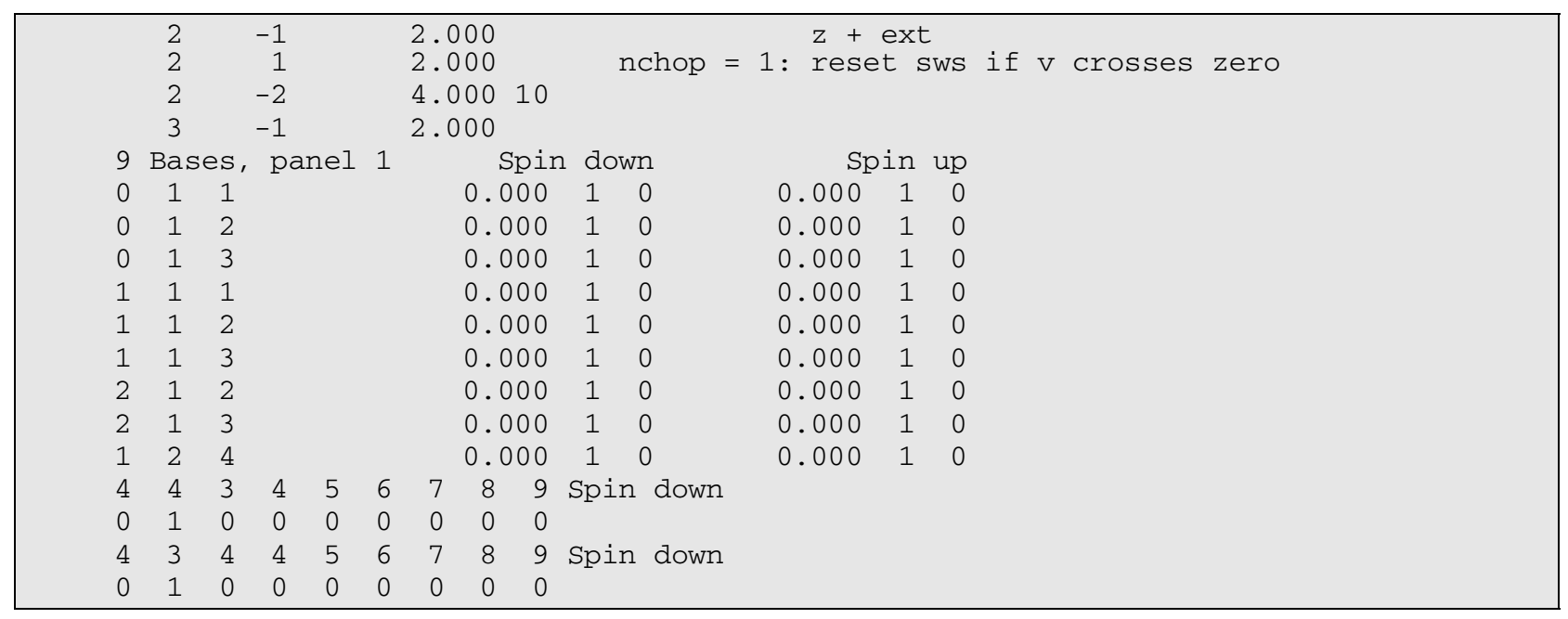




\section{Chapter 4 FP-LMTO calculation of thermal expansion}

\subsection{Introduction}

Electronic structure calculations based on the density functional theorem in the LDA approximation have proved to be an efficient technique for studying zero-temperature energetics in many systems [1]. However, their application to study thermodynamics remains challenging due to the increase of the computational effort required.

The process of thermal expansion is related to the anharmonic behavior of lattice phonons. Therefore, the calculation of the thermal expansion coefficient requires determination of the entire phonon spectrum at a given lattice constant with the high accuracy. It was recently shown that density-functional theory (DFT) based linear response theory can provide a good description of thermal expansion of elemental metals and is capable of calculation phonon spectrum of FeAl at zero temperature $[2,3]$. However, this method has a serious limitation in terms of a size of the computational unit cell - for large number of the atoms the computations become extremely time-consuming. The concentration of the transition metal additives in ironaluminides rarely exceeds $10 \%$ of the total composition that forces us to consider at least $10-20$ atoms in the supercell. Therefore, we are seeking more efficient methodology.

At temperatures comparable with the Debye temperature we use full-potential LMTO method based on the local density approximation (LDA) and incorporated into the Debye model of a solid. This approach has been shown to yield good results for molibdenum-silicides [4] that, like iron-aluminides, have a strong covalent-bonding between the atoms and therefore, the contribution of the optical phonons can be neglected in thermal expansion. 
Although, it is known that LDA gives an erroneous answer in describing the ground state structure of elementary iron and predicts a ferromagnetic ground state for $\mathrm{FeAl}$ compound that was not confirmed by an experiment $[5,6]$. Even though, when studying the thermal expansion one is mostly interested in having an adequate description of elastic properties and that of a phonon spectrum of the material. Here LDA is capable of providing the results that are in a pretty good agreement with the experiment [7].

\subsection{Methodology}

All equilibrium thermodynamic information about a system may be extracted from the free energy and its variation with respect to physical conditions. The free energy at a given temperature has contributions from both the lattice vibrations and thermal excitations of the electrons. At room temperature the latter becomes negligibly small comparing to the energy of the phonons.

One of the most efficient approaches for determining the lattice contributions to the free energy is the quasi-harmonic approximation. In that approximation the Helmholtz free energy of a crystal is defined by the following expression [8]:

$$
F=U(a)+F_{\text {phonons }}=U(a)+k_{b} T \sum_{s} \sum_{k} \ln \left\{2 \sinh \left[\hbar w_{s}(k, a) / 2 k_{b} T\right]\right\},
$$

where the first term is the total energy of the static lattice at a given volume $V$ and $w(k, a)$ is the frequency of the $s$-th phonon at the $k$-point in the Brillouin zone. The anharmonicity of the free energy is included through the explicit volume dependence of the vibrational frequencies.

For a crystal with $p$ ions in a primitive unit cell there are $3 p$ vibrational modes by the number of their degrees of freedom. Three of these modes are called acoustical and the rest of 
them are regarded as optical vibrational modes. In general case to determine the entire phonon spectrum, it is necessary to calculate the Dynamical Matrix [9] for the atomic system and diagonalize it. The calculation of a Dynamical Matrix is computationally very expensive, since it requires consideration of a large supercell in the presence of various atomic displacements. However, in iron-aluminides the optical phonons have much higher activation energy $(\sim 10 \mathrm{THz})$ than the acoustical phonons $(\sim 5 \mathrm{THz})[3]$ and therefore, their contribution into thermal expansion can be neglected at the temperatures comparable with the Debye temperature. Such a property of optical phonons is due to the pronounced covalent bonding between iron and aluminum atoms. In the covalent crystals the bonding strength is significantly higher in some particular directions and therefore, the atoms are more likely to oscillate in the same phase. This let us approximate the entire phonon spectrum of the iron-aluminides by only acoustical frequencies and that what is called the acoustical response approximation or the Debye model.

The acoustical frequencies depend on the elasticity of the lattice and can be calculated from the Christoffel equations [10]:

$$
\left|C_{\mu i \eta j} k_{i} k_{j}-\rho w^{2} \delta_{\mu \eta}\right|=0
$$

where $C_{\mu i j v}$ are elastic constants, $\rho$ is the density of the material and $\omega$ are the acoustic frequencies. To compensate for the neglect of the optical phonons, the integration of the phonon spectrum is carried over the first $p$ Brillouin zones. The volume dependence of the frequencies can be found from the dependence of elastic constants on the lattice volume or so called elastic constants of the third-order. By minimizing the free energy in (4.1) with respect to the volume at different temperatures, one can obtain the value of thermal expansion coefficient. 
The elastic constants of any crystal can be found by distorting the lattice vectors and relaxing all the internal parameters. The lattice vectors under the strain $\varepsilon$ are transformed to the new vectors by the following formulae:

$$
\left(\begin{array}{l}
a_{1}^{\prime} \\
a_{2}^{\prime} \\
a_{3}^{\prime}
\end{array}\right)=\left(\begin{array}{l}
a_{1} \\
a_{2} \\
a_{3}
\end{array}\right)(I+\varepsilon)
$$

where $I$ is the $3 \times 3$ identity matrix. The strain $\varepsilon$ is represented by the symmetric tensor with six independent components:

$$
\mathcal{E}=\left(\begin{array}{ccc}
e_{1} & e_{6} / 2 & e_{5} / 2 \\
e_{6} / 2 & e_{2} & e_{4} / 2 \\
e_{5} / 2 & e_{4} / 2 & e_{3}
\end{array}\right)
$$

Then the total energy of a crystal changes by the following amount:

$$
E=E_{0}-P(V) \Delta V+\frac{V}{2} \sum_{i=1}^{6} \sum_{j=1}^{6} C_{i j} e_{i} e_{j}+O\left[e_{i}^{3}\right]
$$

where $\Delta V$ is the change in the volume due to the strain, $P(V)$ is the pressure of the undistorted lattice at volume $V, C_{i j}$ are the elastic constants. In general case, there are 21 independent elastic constants. However, for cubic crystals this number is reduced to only three elastic constants, namely $\mathrm{C}_{11}, \mathrm{C}_{12}$ and $\mathrm{C}_{44}$. The conditions for a cubic lattice to be stable can be expressed through the following relationships of the elastic constants:

$$
\mathrm{B}=\left(\mathrm{C}_{11}+2 \mathrm{C}_{12}\right) / 3>0, \quad \mathrm{C}_{11}-\mathrm{C}_{12}>0, \quad \mathrm{C}_{44}>0 .
$$

Thus, it would be logical to choose these physically important relationships for elastic constants calculations. 
The bulk modulus of a solid is proportional to a second-order derivative of the total energy with respect to the volume and is defined as follows:

$$
B=-V \frac{\partial^{2} E}{\partial V^{2}}
$$

To calculate the bulk modulus and its dependence on the lattice parameter, we expanded and contracted the lattice vectors within $10 \%$ of the equilibrium lattice parameter. Then we fitted the total energy curve into the function of the form:

$$
P(V)=e_{0}-2 e_{1} \exp \left(-\alpha \frac{V_{0}}{V}\right)+e_{2} \exp \left(-2 \alpha \frac{V_{0}}{V}\right),
$$

where $\mathrm{e}_{0}, \mathrm{e}_{1}, \mathrm{e}_{2}, \alpha$ are unknown parameters and $\mathrm{V}_{0}$ is the equilibrium volume, and calculated the second-order derivatives at several different lattice parameters.

To find $\mathrm{C}_{11}$ and $\mathrm{C}_{12}$ difference, we followed Mehl et al [11] and considered the volumeconserving orthorombic deformation:

$$
\begin{aligned}
& x^{\prime}=x(1+\Delta) \\
& y^{\prime}=y(1-\Delta) \\
& z^{\prime}=z\left(1-\frac{\Delta^{2}}{1-\Delta^{2}}\right)
\end{aligned}
$$

For this deformation, the energy of a crystal is represented by an even function of the strain:

$$
E=E_{0}(a)+V\left(C_{11}(a)-C_{12}(a)\right) \Delta^{2}+O\left[\Delta^{4}\right]
$$

The conservation of the volume in the elastic constants calculations has a big advantage, since the pressure does not contribute to the energy change. At the same time, the term after the second one in the polynomial expansion of the energy is of the fourth order that increases the accuracy of the calculations. 
To obtain the third-order elastic constants, namely $\mathrm{C}_{111}-\mathrm{C}_{112}$, we performed the calculations of $\mathrm{C}_{11}-\mathrm{C}_{12}$ at several different lattice parameters and differentiated $\mathrm{C}_{11}-\mathrm{C}_{12}$ with respect to the lattice parameter.

In order to find $\mathrm{C}_{44}$, we considered the following shear deformation:

$$
\begin{aligned}
& x^{\prime}=x+\Delta y \\
& y^{\prime}=y+\Delta x \\
& z^{\prime}=z\left(1+\frac{\Delta^{2}}{1-\Delta^{2}}\right)
\end{aligned}
$$

with the energy change given by $E=E_{0}(a)+2 V C_{44}(a) \Delta^{2}+O\left[\Delta^{4}\right]$;

Then, same procedure as described for $\mathrm{C}_{11}-\mathrm{C}_{12}$ was repeated. Again, from six to ten different lattice constants were considered to calculate $\mathrm{C}_{441}$.

The total energy of the static lattice and the elastic constants were calculated by our FPLMTO method described in details in Chapter 2.

\subsection{Results}

We started our research by calculating the lattice parameters and the elastic constants of pure FeAl and $\mathrm{Fe}_{3} \mathrm{Al}$. The results of calculations are presented in Table 4.1. For both ironaluminides the equilibrium lattice constants are at least 3\% smaller than the one measured experimentally that is typical for LDA dealing with the iron compounds. The calculated lattice parameter and the elastic constants of $\mathrm{FeAl}$ are found to be in a good agreement with the previous work done by C.L.Fu et al using full-potential linearized augmented plane-wave (FPAPW) method as a computational technique [12].

One of the most important properties of the elastic constants is the negative Caushi discrepancy, defined as $\mathrm{C}_{12}-\mathrm{C}_{44}$. It indicates that the shear deformation of a crystal requires more 
energy than its expansion or contraction in any direction at the same absolute value of the strain. Thus, the negative Caushi discrepancy is typical for covalent crystals and FP-LMTO calculations reproduce it with the reasonable accuracy. However, for both iron-aluminides the absolute values Table 4.1 Equilibrium lattice constants and elastic constants of $\mathrm{FeAl}$ and $\mathrm{Fe}_{3} \mathrm{Al}$. Lattice parameter $\mathrm{a}_{0}$ is in $\mathrm{A}$, elastic constants are in GPa.

\begin{tabular}{|c|c|c|c|c|c|}
\hline & $\mathrm{a}_{0}$ & $\mathrm{C}_{11}$ & $\mathrm{C}_{12}$ & $\mathrm{C}_{44}$ & $\mathrm{C}_{12}-\mathrm{C}_{44}$ \\
\hline $\mathrm{FeAl}$ & 2.81 & 281 & 140 & 167 & -27 \\
\hline Fu et $\mathrm{al}^{\mathrm{a}}$ & 2.83 & 290 & 130 & 165 & -35 \\
\hline Exp. $^{b}$ & 2.90 & 181 & 114 & 127 & -13 \\
\hline $\mathrm{Fe}_{3} \mathrm{Al}$ & 5.50 & 272 & 210 & 196 & 14 \\
\hline Exp. $^{c}$ & 5.79 & 171 & 131 & 132 & -1 \\
\hline
\end{tabular}

${ }^{\mathrm{a}}$ Reference 12

${ }^{\mathrm{b}}$ Reference 13

${ }^{\mathrm{c}}$ Reference 14

of elastic constants are much higher than the experimental results. Here, we have to specify three major sources of errors present in our calculations.

The elastic constants listed in the Table 4.1 were measured at room temperature, while FP-LMTO technique allows one to calculate the energy of the system found in the ground state. Therefore, the thermal vibrations of the atoms were neglected in this method. To see how big is their contribution into the elastic properties, we performed the calculation of the bulk modulus of $\mathrm{Fe}_{3} \mathrm{Al}$ using the Debye model at room temperature. In the Figure 4.1 the energy-volume curve at room temperature is plotted together with the one obtained from zero-temperature LDA calculations. The solid curve corresponds to zero-temperature uniform expansion, while the dashed curve shows the volume dependence of the total energy at room temperature. The 
equilibrium lattice parameter at a given temperature is determined by a position of the minimum of the energy-volume curve and the bulk modulus is measured from the local curvature at the minimum.

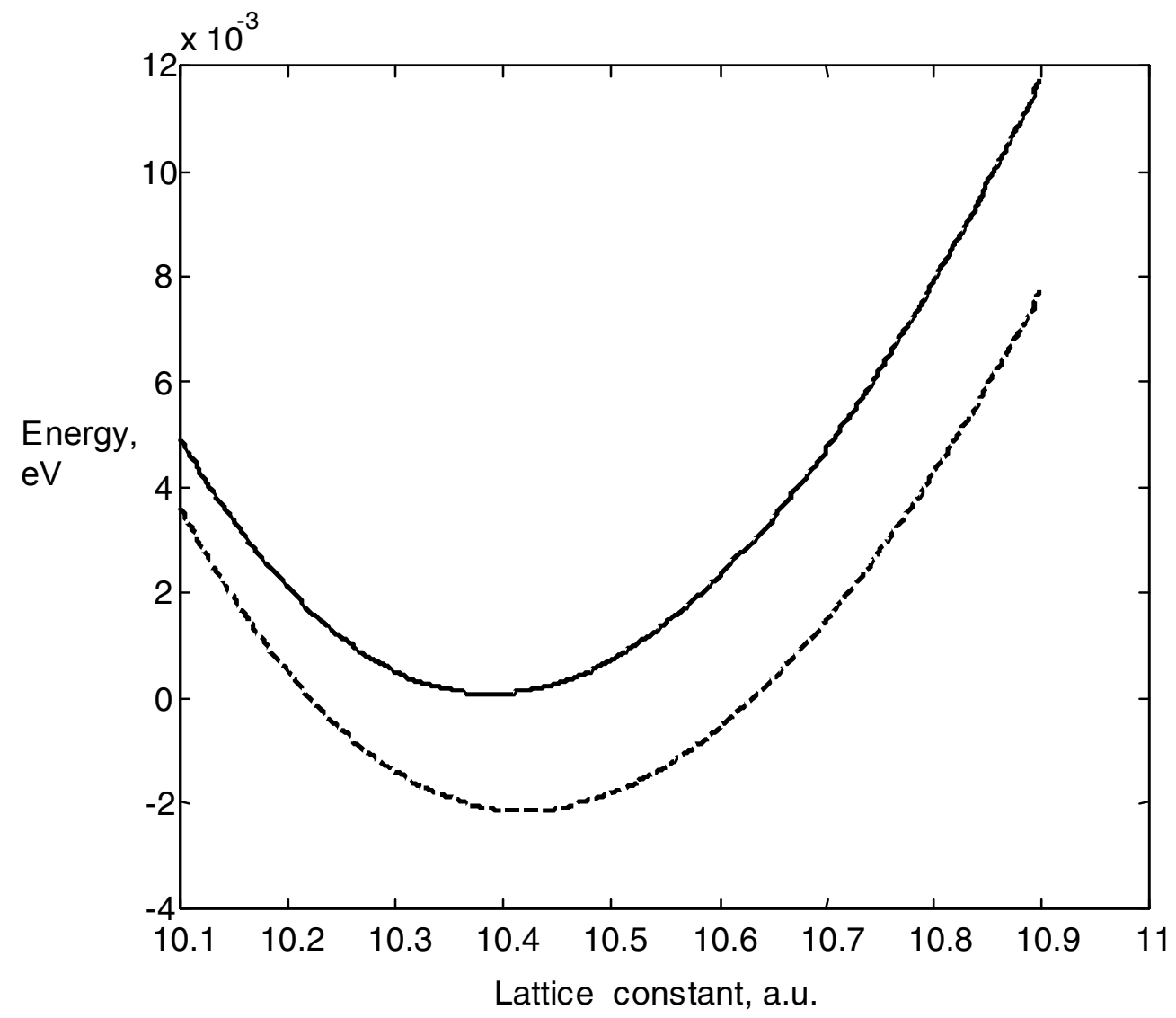

Figure 4.1. Equation of state for $\mathrm{Fe}_{3} \mathrm{Al}$. The solid curve is the total energy calculated by LDA at $\mathrm{T}=0 \mathrm{~K}$ and the dashed curve is the Helholtz free energy at $\mathrm{T}=293 \mathrm{~K}$

While the effect of the phonons on the equilibrium lattice constant is noticeably strong, indicating the thermal expansion of the crystal, the inclusion of the lattice vibrations slightly modifies the value of the bulk modulus. Thus, at a room temperature for $\mathrm{FeAl}$ and $\mathrm{Fe}_{3} \mathrm{Al}$ it increases about $3 \mathrm{GPa}$ that is of the order of the computational noise present in the calculations. In Figure 4.2 we plotted the dependence of the bulk modulus on the lattice parameter calculated 
from the static total energy received from LDA calculations and from the temperature-dependent Helmholtz free energy (4.1). One can see that indeed, the deviation of the two curves becomes significant only at large lattice constants that correspond to high temperatures.

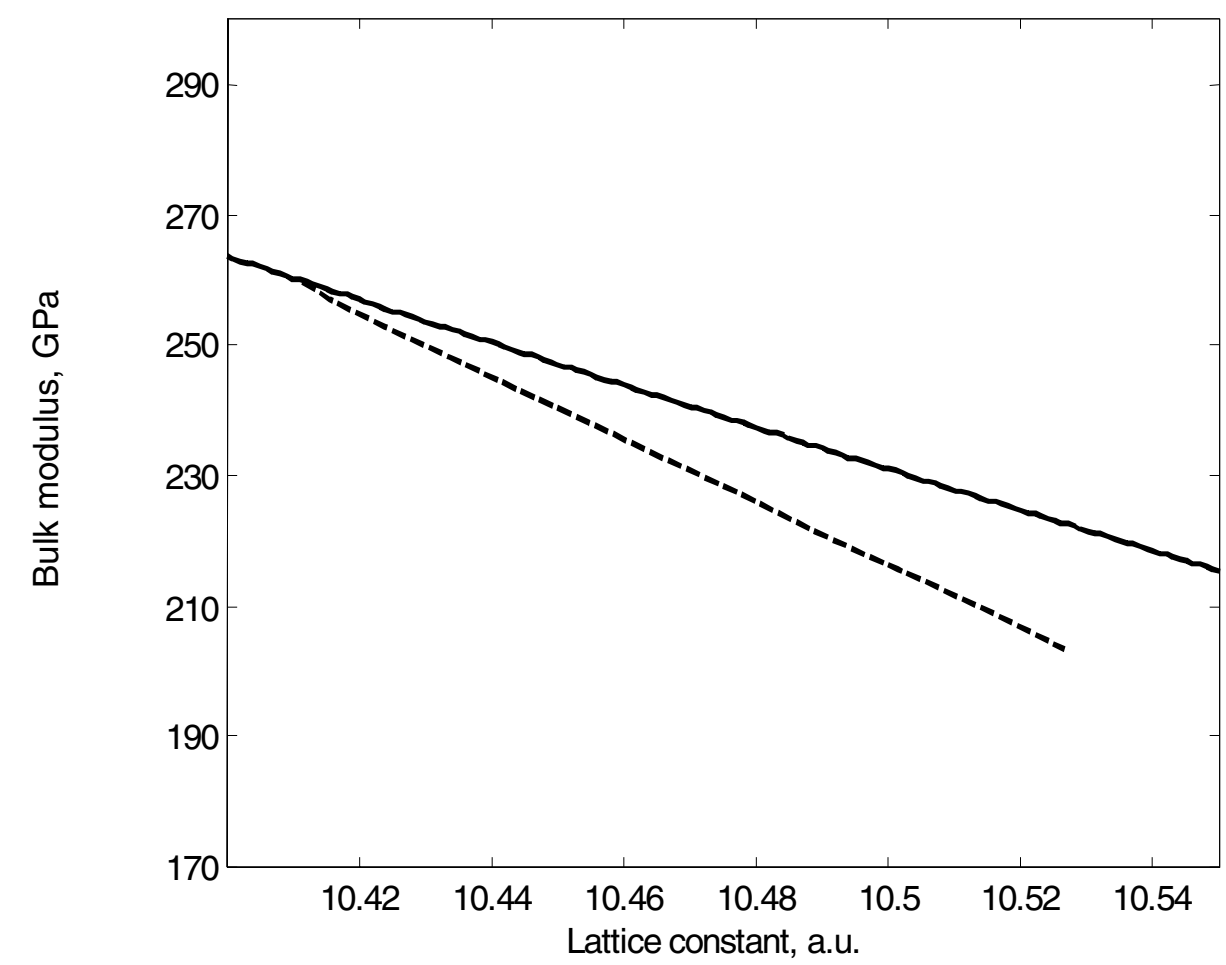

Figure 4.2 Calculated bulk modulus as a function of lattice parameter for $\mathrm{Fe}_{3} \mathrm{Al}$. The solid curve is obtained from the temperature-dependent free energy and the dashed curve is obtained from $\mathrm{T}=0$ electronic total energy.

A second source of errors presented in our calculations was the fact that we completely ignored the relaxation of the atoms in the lattice under the strain. This relaxation could lower the value of the total energy of the lattice and therefore decrease the values of the elastic constants. The relaxation becomes particularly important for $\mathrm{Fe}_{3} \mathrm{Al}$, since it is found in $\mathrm{DO}_{3}$ structure and therefore, it possesses 16 atoms in a supercell with the cubic symmetry. Studying the influence 
of the relaxation on the elastic constants would require repeated calculations for a supercell with the distorted symmetry that are not feasible to perform by FP-LMTO method. Therefore, we studied that issue using the many-body atomistic potentials inferred from the FP-LMTO total energy calculations. The results are presented in the Chapter 5.3. Here, we want to mention that the inclusion of the atomic relaxation lowers the value of the elastic constants up to $10 \mathrm{GPa}$.

The last source of errors refers to the Local Density Approximation (LDA) itself. It is known to overestimate the bonding between the atoms. It was suggested by Mehl et al [11] that in order to achieve a better agreement with the experiment, the elastic constants have to be calculated at the experimental lattice constant of the material rather than the one calculated by LDA. In Table 4.2 we present the elastic constants of $\mathrm{FeAl}$ and $\mathrm{Fe}_{3} \mathrm{Al}$ calculated by this approach. One can see that indeed the values of the elastic constants decrease, hence getting closer to their experimental values. Nevertheless, our final goal is to calculate the thermal expansion of iron-aluminides from the first-principles without any experimental input, therefore in our calculations we were using the values of elastic constants presented in the Table 4.1. At the same time the approach of Mehl et al is hardly applicable to the intermetallic compounds in the presence of additives, since the addition of transition metal elements changes both the theoretical and experimental values of equilibrium lattice constant sometime in a different way.

The third-order elastic constants are presented in Table 4.3. There is no experimental data available for comparison. 
Table 4.2 Elastic constants of $\mathrm{FeAl}$ and $\mathrm{Fe}_{3} \mathrm{Al}$ calculated at the experimental lattice constants. Experimental lattice parameter $\mathrm{a}_{0}$ is in $\mathrm{A}$, elastic constants are in GPa.

\begin{tabular}{|c|c|c|c|c|}
\hline & $a_{\exp }$ & $\mathrm{C}_{11}$ & $\mathrm{C}_{12}$ & $\mathrm{C}_{44}$ \\
\hline $\mathrm{FeAl}$ & 2.90 & 190 & 114 & 139 \\
\hline Mehl et al ${ }^{\mathrm{a}}$ & 2.90 & 182 & 78 & 45 \\
\hline Exp. $^{b}$ & 2.90 & 181 & 114 & 127 \\
\hline $\mathrm{Fe}_{3} \mathrm{Al}$ & 5.79 & 163 & 129 & 117 \\
\hline Exp. $^{\mathrm{c}}$ & 5.79 & 171 & 131 & 132 \\
\hline
\end{tabular}

${ }^{\mathrm{a}}$ Reference 11

b Reference 13

${ }^{\mathrm{c}}$ Reference 14

Table 4.3 Calculated third-order elastic constants in GPa.

\begin{tabular}{|c|c|c|c|}
\hline & $\mathrm{C}_{111}$ & $\mathrm{C}_{121}$ & $\mathrm{C}_{441}$ \\
\hline $\mathrm{FeAl}$ & -3689 & -2132 & -2367 \\
\hline $\mathrm{Fe}_{3} \mathrm{Al}$ & -3907 & -3149 & -1899 \\
\hline
\end{tabular}

Even though, the elastic constants of $\mathrm{FeAl}$ and $\mathrm{Fe}_{3} \mathrm{Al}$ differ from the experiment, the calculated linear thermal expansion for both aluminides is in a good agreement with the results of the measurements as it is shown on the Figures 4.3 (a) and (b) for $\mathrm{FeAl}$ and $\mathrm{Fe}_{3} \mathrm{Al}$ respectively. The thermal expansion coefficient in case of the temperature independent bulk modulus can be represented as follows [15]:

$$
\alpha=\frac{1}{3 B} \sum_{k s}\left(-\frac{\partial}{\partial V} h \omega_{k s}\right) \frac{\partial}{\partial T} n_{s} \quad(k)
$$


Thus, it mostly depends on the relationship between the unharmonicity of the phonons and the elasticity of the crystal. The obtained good agreement of our calculations with the experiment indicates that the errors in determining these two properties are of the same order and therefore, cancel out each other during the calculations or one can also argue that the LDA overestimates the elastic constants of iron aluminides, it reproduces the relationship on the right-hand side of (4.6) in the appropriate agreement with the experiment. In particular, an exceptionally good agreement was obtained for linear thermal expansion of $\mathrm{Fe}_{3} \mathrm{Al}$. The theoretical results start to deviate from the experimental only at temperatures higher than $600 \mathrm{~K}$. Such a deviation is certainly understandable, since $\mathrm{Fe}_{3} \mathrm{Al}$ is undergoing a second-order phase transition at temperatures around $800 \mathrm{~K}$. In our calculations $\mathrm{Fe}_{3} \mathrm{Al}$ is considered to be a perfect crystal in ordered $\mathrm{DO}_{3}$ for all temperature ranges. 
Figure 4.3 (a, b) Temperature dependence of the linear thermal expansion of $\mathrm{FeAl}$ and $\mathrm{Fe}_{3} \mathrm{Al}$. The solid line connects the results of calculations and the triangles represent the experimental data from [16].

(a)
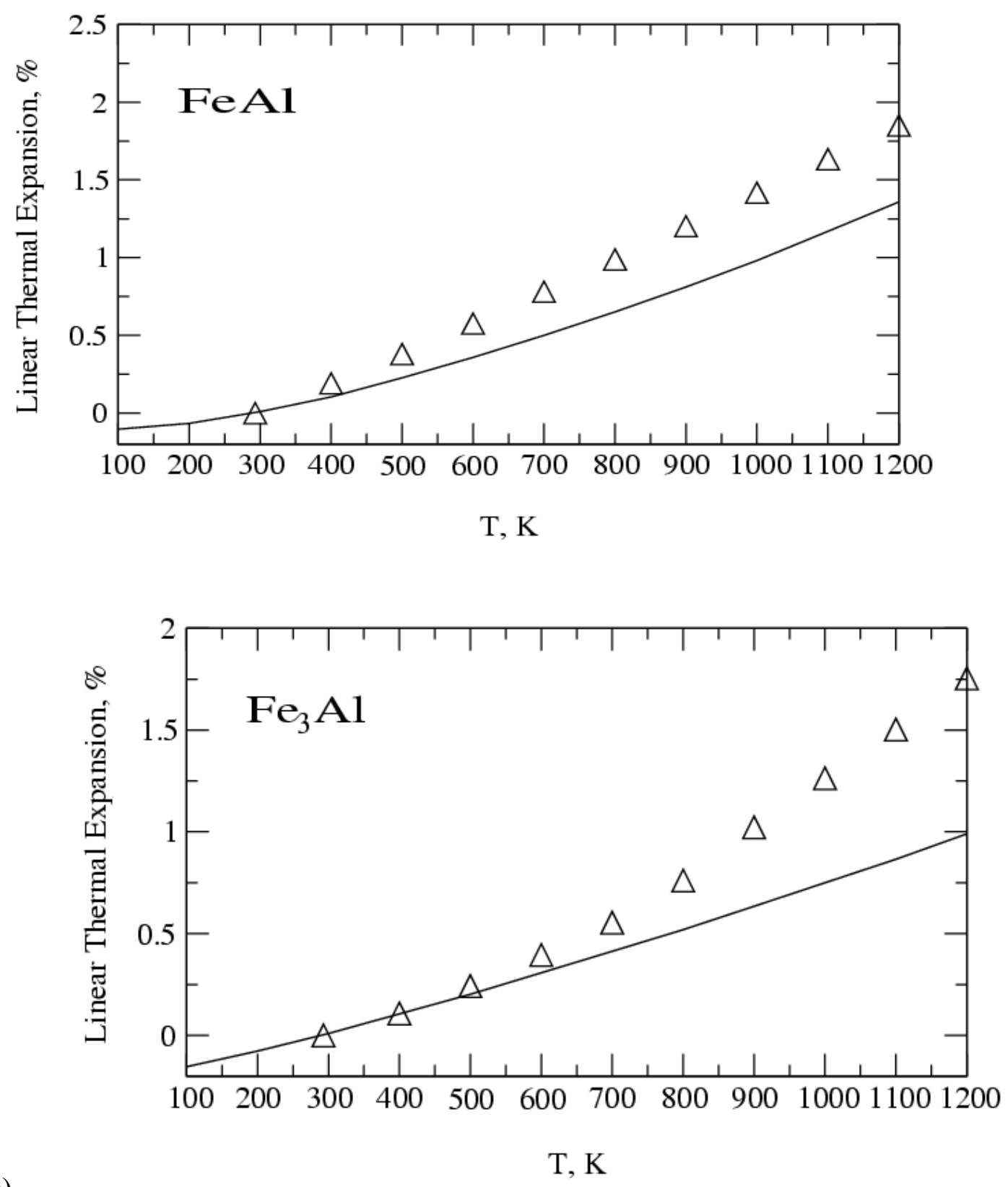

(b) 
After calculating the thermal expansion of pure aluminides, we attempted to apply the same methodology for calculating the thermal expansion of $\mathrm{Fe}_{3} \mathrm{Al}$ with the addition of Mo and $\mathrm{V}$. We considered only one possible concentration of these transition metal elements in $\mathrm{Fe}_{3} \mathrm{Al}$, namely $6.25 \%$ of the iron content substituted by these transition metal elements. Our computational supercell of $\mathrm{Fe}_{3} \mathrm{Al} \mathrm{DO}_{3}$ structure had 16 basis atoms in the cubic lattice presented in the Figure 3.1. The atom of Mo and later V atom was placed at FeI site, since this lattice site is the most energetically favorable according to our calculations in Chapter 3. Since our FP-LMTO calculations are very time-consuming, we did not study the effect of randomness of the additive distribution on the thermal expansion of the compound.

The calculated lattice constants together with the elastic constants are presented in the Table 4.4. This table shows that the iron substitution by either Mo or V leads to the lattice volume expansion. While in the case of Mo additive the relative change of the lattice parameter is in a good agreement with the experiment [17], the measurements show that vanadium substitution up to the $33 \%$ of the iron content results in a significant lattice contraction [17]. The atomic radius of vanadium is larger than that of iron, therefore, the observed contraction can be presumably referred to enhanced cohesion by the $\mathrm{DO}_{3}$-type ordering or, in other words, the $\mathrm{V}$ substitution

Table 4.4 Elastic constants of $\mathrm{Fe}_{3} \mathrm{Al}$ with the addition of Mo and V. Lattice parameter $\mathrm{a}_{0}$ is in $\mathrm{A}$, elastic constants are in GPa.

\begin{tabular}{|c|c|c|c|c|c|}
\hline & $a_{0}$ & $C_{11}$ & $C_{12}$ & $C_{44}$ & $C_{12}-C_{44}$ \\
\hline $\mathrm{Fe}_{3} \mathrm{Al}$ & 5.50 & 272 & 210 & 196 & 14 \\
\hline$\left(\mathrm{Fe}_{0.917} \mathrm{Mo}_{0.083}\right)_{3} \mathrm{Al}$ & 5.58 & 325 & 196 & 148 & 48 \\
\hline$\left(\mathrm{Fe}_{0.917} \mathrm{~V}_{0.083}\right)_{3} \mathrm{Al}$ & 5.53 & 343 & 188 & 153 & 35 \\
\hline
\end{tabular}


referred to enhanced cohesion by the $\mathrm{DO}_{3}$-type ordering or, in other words, the $\mathrm{V}$ substitution for iron may lead to the decrease of the number of

the open-volume defects. However, in our FP-LMTO calculations we are using a perfect monocrystal model for the intermetallic compound and thus, it is impossible to test the previous statement within our methodology.

The addition of Mo and $\mathrm{V}$ in $\mathrm{Fe}_{3} \mathrm{Al}$ produces the same effect on the elastic constants. While the value of $C_{44}$ decreases after the substitution of these transition metals, both $C_{11}$ and $C_{12}$ elastic constants significantly increase indicating the growing cohesion of the $\mathrm{DO}_{3}$ structure ordering. Another important feature is that the Caushi discrepancy, $C_{12}-C_{44}$, becomes smaller. This is related to the fact that the bonding between the atoms becomes more uniform in all directions. The third-order elastic constants are listed in the Table 4.5.

Table 4.5 Calculated third-order elastic constants in GPa of $\mathrm{Fe}_{3} \mathrm{Al}$ with Mo and V additives.

\begin{tabular}{|c|c|c|c|}
\hline & $\mathrm{C}_{111}$ & $\mathrm{C}_{121}$ & $\mathrm{C}_{441}$ \\
\hline $\mathrm{Fe}_{3} \mathrm{Al}$ & -3907 & -3149 & -1899 \\
\hline$\left(\mathrm{Fe}_{0.917} \mathrm{Mo}_{0.083}\right)_{3} \mathrm{Al}$ & -2840 & -1580 & -1130 \\
\hline$\left(\mathrm{Fe}_{0.917} \mathrm{~V}_{0.083}\right)_{3} \mathrm{Al}$ & -4490 & -2720 & -1245 \\
\hline
\end{tabular}


The thermal expansion of $\mathrm{Fe}_{3} \mathrm{Al}$ with the addition of Mo and $\mathrm{V}$ elements is presented on Figure 4.4. As seen in this Figure, the addition of Mo decreases the thermal expansion of $\mathrm{Fe}_{3} \mathrm{Al}$, while addition of $\mathrm{V}$ leads to a larger thermal expansion. However, the effect produced by both transition elements is relatively small and becomes noticeable only at elevated temperatures. Thus, at a temperature $1200 \mathrm{~K}$, the difference in linear thermal expansion of pure $\mathrm{Fe}_{3} \mathrm{Al}$ and with transition metal additives reaches $\sim 15 \%$ that resides close to the border of the computational accuracy of our method. Since the computational error in determining elastic constants was about $5 \%$ of their absolute value, the third-order elastic constants were calculated within $10 \%$ of the relative error. The same computational accuracy is expected in the thermal expansion calculation. One can conclude that more accurate computational approach is necessary to capture the effect of the transition metal additives on the thermal expansion of iron-aluminides. At the same time the addition of transition metal elements can lead to a substantial change in the phonon spectrum that makes the use of the Debye model inappropriate to these materials. An alternative ab initio based approach to thermal expansion studies is molecular dynamics simulations using many-body potentials. These computational results are presented in Chapter 5. 
Figure 4.4 The effect of the Mo and V additives on the thermal expansion of $\mathrm{Fe}_{3} \mathrm{Al}$. The solid line connects the calculated values of thermal expansion of pure $\mathrm{Fe}_{3} \mathrm{Al}$. The circles represent the thermal expansion of $\left(\mathrm{Fe}_{0.917} \mathrm{Mo}_{0.083}\right)_{3} \mathrm{Al}$, and triangles denote $\left(\mathrm{Fe}_{0.917} \mathrm{~V}_{0.083}\right)_{3} \mathrm{Al}$ linear thermal expansion.

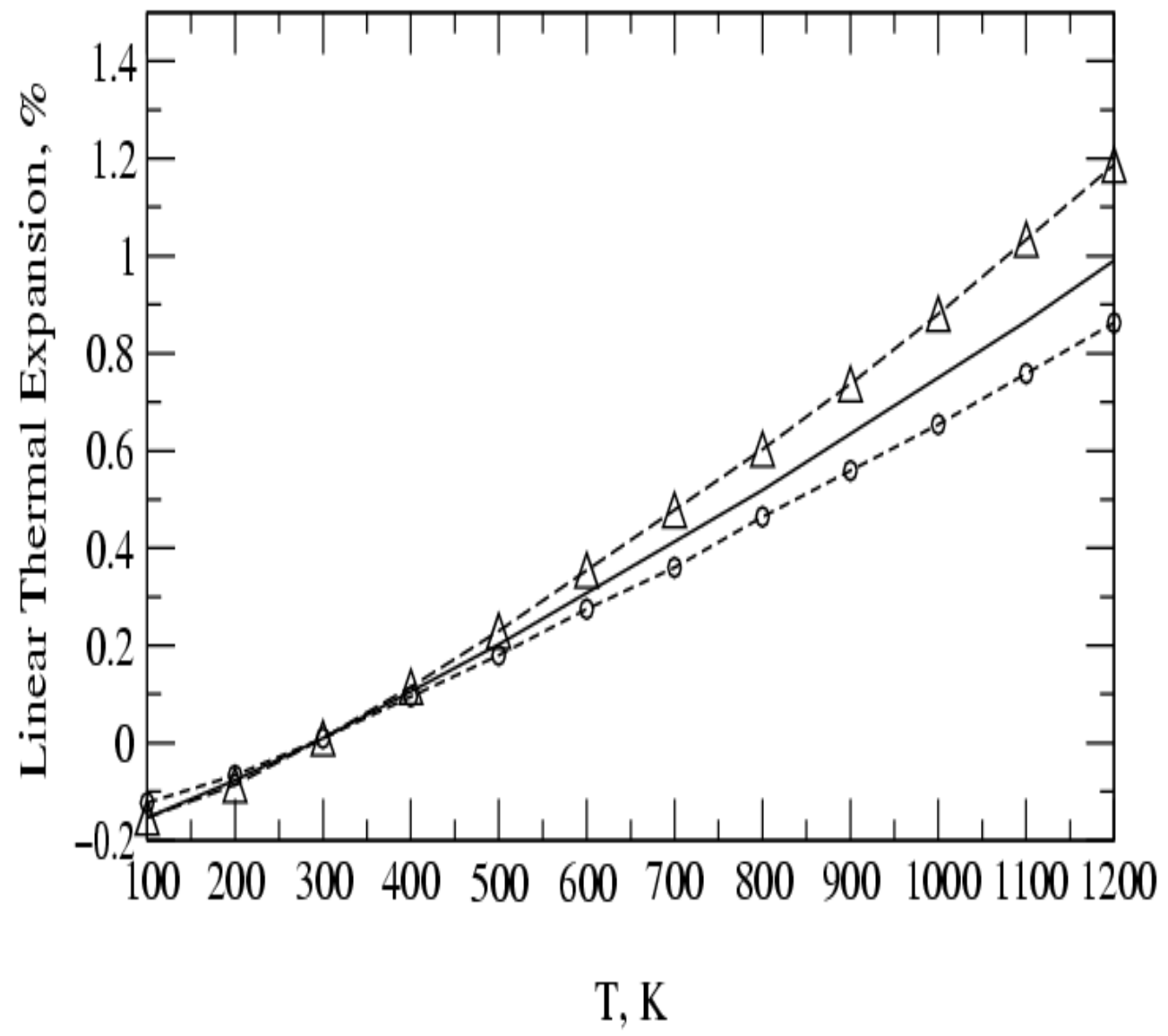




\section{References:}

1. W.E.Pickett, Comput.Phys.Rep.,vol.9, 115 (1989)

2. A.A.Quong, A.Y.Liu, Phys Rev B, vol.56, 13, p.7767-70, (1997)

3. B.Meyer, V.Scott, M.Fahnle, Phys. Rev. B, vol. 58, 22 (1998)

4. C.L.Fu and Xindong Wang, Philosophical Magazine Letters 80, 10, (2000)

5. W.E.Pickett, Comput.Phys.Rep.,vol.9, 115 (1989)

6. B.Meyer, V.Scott, M.Fahnle, Phys. Rev. B, vol. 58, 22 (1998)

7. F.Lechermann, F.Welsch, C.Eslasser, C.Ederer, M.Fahnle, J.M.Sanchez, B.Meyer, Phys. Rev. $B$, vol.65, p.132104-1 - 4, (2002)

8. A.A.Maradudin, E.W.Montroll, G.H.Weiss, I.P.Ipatova, Theory of Lattice Dynamics in Harmonic Approximation, $2^{\text {nd }}$ ed., (Academic, New York, 1971)

9. See for example, P.Pavone, K.Karch, O.Schutt, W.Windl, D.Strauch, P.Giannozi, S.Baroni, Phys.Rev. B, vol.48, 5, pp.3156-3163 (1993)

10. N.W.Ashcroft, N.D.Mermin, Solid State Physics (Holt,Rindhart and Winston) p.447 (1976)

11. M.J.Mehl, B.M.Klein, D.A.Papaconstantopoulos, Intermetallic Compounds:Principles and Practise, vol.I: Principles, J.H.Westbrook, R.L.Fleischer, eds., John Wiley and Sons, pp.195-210 (London 1995),

12. C.L.Fu, M.H.Yoo, Acta metal.mater., vol.40, No.4, pp.703-711 (1992)

13. G.Simmons, H.Wang, Single Crystal Elastic Constants and Calculated Aggregate Properties (M.I.T.Press, Boston, 1971) 
14. H.J.Leamy, E.D.Gibson, F.X.Kayser, Acta Metalurgica vol.15, pp.1827-1838, December (1967)

15. N.W.Ashcroft, N.D.Mermin, Solid State Physics (Holt,Rindhart and Winston) p.447, (1976)

16. Y.S.Touloukian, R.K.Kirby, R.E.Taylor, P.D.Desai, Thermodynamical Properties of matter: Thermal Expansion Metallic Elements and Alloys, ( IFI/ Plenum, NY 1975)

17. Y.Nishino, S.Asano, T.Ogawa Material Science and Engineering, A234-236, p.271-274, (1997) 


\section{Chapter 5}

\section{Molecular dynamics simulation of thermal expansion of iron- aluminides in the presence of additives}

\subsection{Introduction}

Classical Molecular Dynamics (MD) simulation is a powerful and practical tool for studying thermodynamical properties of many-particle systems. It is especially valuable for calculation of thermal expansion at high temperatures. When the temperature of a crystal rises, the kinetic energy of the atoms grows and additional vibrational modes of the lattice are getting excited. At the same time the motion of the atoms ceases to be harmonic indicating that the Debye approximation is not applicable any more. When studying the behavior of the atomic system at high temperatures, it is necessary to consider all possible degrees of freedom of the atoms and there, MD provides an efficient computational technique for integrating the equations of motion.

An important issue is the way the interatomic forces are calculated in the Molecular Dynamics algorithm. Since ab initio calculations are extremely time-consuming, many-body atomistic potentials are the ones most frequently used in these simulations. For iron-aluminides we found two parameterization schemes of the interatomic interaction in the literature. One of them was proposed by Besson et al [1]. The parameters of interaction between the atoms of the same type (Fe-Fe interaction, Al-Al) were designed to reproduce the selected bulk properties of pure materials measured experimentally. At the same time the parameters of interaction between 
$\mathrm{Fe}$ and $\mathrm{Al}$ atoms were fitted into the elastic constants and vacancy formation energies of FeAl obtained from the first principles. Even though the majority of physical quantities calculated from these potentials are in a reasonably good agreement with the experiment, this way of calculating the interatomic potentials is not self-consistent, since depending on the type of the material the potentials were fitted either to experimental or computations. Therefore, we are looking for another parameterization scheme.

Another approach was suggested by Wen-qing Zhang et al [2]. They used the lattice inversion method to calculate the vibrational entropies of ordered and disordered ironaluminides. The lattice inversion method does calculate the pair potential parameters analytically from the Rose universal equation of state [3]. The pair potentials have spherical symmetry and do not depend on the angles between the atoms in the lattice. The iron-aluminum compounds are known to have a strong directional bonding between the iron and aluminum atoms, but the pair potentials are not capable of reproducing this important property of iron-aluminides. At the same time, the parameters obtained from the lattice inversion method are by definition nontransferable between the different structures of one and the same compound. That is particularly important for $\mathrm{Fe}_{3} \mathrm{Al}$, since in this material a second-order phase transition from $\mathrm{DO}_{3}$ to $\mathrm{B}_{2}$ structure takes place at 550C [4].

Our many-body atomistic potentials are based entirely on the first-principles calculations. They represent an effective tool for dynamic study of transition metal alloys and were previously successfully applied to nickel binary alloys [5] and NiAl compound [6]. Our model starts from a formal expansion of total energy provided by generalized pseudo-potential theory [7]. The form of the interatomic potential is chosen to be simple and flexible to include both $\mathrm{FeAl} \mathrm{B}_{2}$ phase and $\mathrm{Fe}_{3 \mathrm{~A}} \mathrm{l} \mathrm{DO}_{3}$ phase into the fitting. The total energy of a crystal is expressed in terms of two- and 
three-body interactions. More importantly, we have introduced a simple local variable function, which transforms a volume-dependent energy part into many-body interaction. The potential parameters were obtained by fitting the energy surfaces resulting from FP-LMTO calculations [8]. The energy surfaces were generated by various elastic deformations of $\mathrm{FeAl}$ and $\mathrm{Fe}_{3} \mathrm{Al}$ lattices. To test our potentials, we considered relative phase stability of $\mathrm{DO}_{3}$ and $\mathrm{B}_{2}$ phases, the formation energies of the defects and thermal expansion of elemental metals. After that we studied the dependence of thermal expansion of $\mathrm{Fe}_{3} \mathrm{Al}$ on different concentrations of Molybdenum and Vanadium.

\subsection{Methodology}

\subsubsection{Molecular Dynamics}

Molecular dynamics is a popular simulation technique for studying thermodynamical properties of molecular and atomic systems. It is based on solving the classical equations of motion for a system of $N$ particles interacting via potential $V$. These equations can be written as follows:

$$
m_{i} r^{\prime \prime}{ }_{i}=f_{i}
$$

where $m_{i}$ designates the mass of atom $i$ and

$$
f_{i}=-\nabla_{r_{i}} V
$$

is the force acting on this atom. Also known as Newtonians equations, (5.1) represents merely a system of ordinary differential equations. A standard method of solving them is the finite 
difference method. The general idea can be described as follows: for given atomic positions, velocities and other dynamical variables at time $t$, obtain the positions, velocities etc at a later time $t+d t$ to a sufficient degree of accuracy. The equations are solved in the incremental fashion. The value of $d t$ depends on which particular algorithm was used to solve the equations, but in any case it has to be significantly smaller than the relaxation time of the system. Among different methods available in literature, we have chosen a Gear predictor-corrector algorithm [9].

If the classical trajectory of the atoms is continuous, then an estimate of the positions and velocities at time $t+d t$ can be found from the Taylor expansion about time $t$ :

$$
\begin{aligned}
& r^{p}(t+d t)=r(t)+d t v(t)+\frac{1}{2} d t^{2} a(t)+\frac{1}{6} d t^{3} b(t)+\ldots \\
& v^{p}(t+d t)=v(t)+d t a(t)+\frac{1}{2} d t^{2} b(t)+\ldots \\
& a^{p}(t+d t)=a(t)+d t b(t)+\ldots \\
& b^{p}(t+d t)=b(t)+\ldots
\end{aligned}
$$

The superscript " $p$ " marks the predicted values, while $\boldsymbol{r}$ and $\boldsymbol{v}$ stand for a complete set of the positions and the velocities, $\boldsymbol{a}$ serves as an abbreviation for all the accelerations and $\boldsymbol{b}$ denotes all third-order time derivatives of $\boldsymbol{r}$. Equations (5.3) provide a suitable way of calculating the atomic coordinates and their derivatives from one time step to the next. However, the generated trajectories will deviate from the true ones with time, unless we choose to ignore the equations of motion. These equations are included in the correction step. From the "predicted" coordinates, it's possible to recalculate new atomic forces and hence, acquire the correct accelerations $a^{c}(t+d t)$. By comparing them with the predicted accelerations $a^{p}(t+d t)$, we get an estimate of the error in the prediction step:

$$
\Delta a(t+d t)=a^{c}(t+d t)-a^{p}(t+d t)
$$


This error and the results from the predictor step are further used to obtain the new positions and the derivatives from the following equations:

$$
\begin{aligned}
r^{c}(t+d t)=r^{p}(t)+c_{0} \Delta a(t+d t) \\
v^{c}(t+d t)=v^{p}(t)+c_{1} \Delta a(t+d t) \\
a^{c}(t+d t)=a^{p}(t)+c_{2} \Delta a(t+d t) \\
b^{c}(t+d t)=b^{p}(t)+c_{3} \Delta a(t+d t)
\end{aligned}
$$

The corrected positions and velocities provide a better approximation of the true atomic trajectories. The choice of $\mathrm{c}_{0}, \mathrm{c}_{1}, \mathrm{c}_{2}, \mathrm{c}_{3}$ coefficients generally depends on the order of polynomial used in Taylor expansion. It also has to satisfy the conditions of optimum stability and accuracy of the trajectories. In our calculations we considered the fourth-order polynomial expansion in (5.1) while solving the equations of motion of the first-order. Thus, the coefficients in (5.3) had the following values:

$$
\mathrm{c}_{0}=3 / 8, \quad \mathrm{c}_{1}=1, \quad \mathrm{c}_{2}=3 / 4, \quad \mathrm{c}_{3}=1 / 6 .
$$

By repeating the corrector steps an appropriate number of times, one will eventually arrive at the iterative solution of Newtonian equations at a time $t+d t$. However, in practice the most time-consuming part of molecular dynamics simulation is the evaluation of accelerations from the particle positions. Since this procedure is repeated at every corrector step, a large number of corrector iterations can become very time-consuming. Therefore, only one corrector step is usually carried out for each predictor step.

Although, the MD algorithms are proven to be highly accurate and stable, it is unreasonable to expect that such an algorithm will dutifully generate the exact classical trajectory. For example, any two classical trajectories that are initially very close will eventually 
diverge from one another with time. This is demonstrated in Figure 5.1. We considered the timedependence of the average distance in the configurational space between two trajectories that is defined as follows:

$$
\Delta r^{2}=(1 / N) \sum\left|r_{i}(t)-r_{i}^{0}(t)\right|^{2}
$$

where $\mathrm{N}$ is the number of particles, $r_{i}^{0}(t)$ is the position of ith atom in the reference simulation and $r_{i}(t)$ is the position of the same atom at the same time in the perturbed simulation. As seen in Figure 5.1, $\Delta r^{2}$ grows exponentially with time reaching the mean-square displacement of the atoms at approximately the $40^{\text {th }}$ time step. However, working with the molecular dynamics, the calculation of exact trajectories is not the main goal. These simulations are usually designed to calculate the exact time-correlation functions and to generate states sampled from the microcanonical ensemble. The particle trajectories must stay on the appropriate constant-energy hypersurface in phase-space, otherwise correct ensemble averages will not be accumulated. Thus, a big emphasis has to be placed on energy and momentum conservation. In the Figure 5.2 the time-dependence of the average energy is represented for two trajectories which relative divergence was shown in the Figure 5.1. One can see that the two energy curves do coincide approaching with time some constant value.

To calculate thermal expansion of iron-aluminides we considered NTP ensemble. A simple way of keeping the temperature of a crystal constant in MD is to rescale the velocities at each time step by a factor of $\sqrt{\frac{T_{0}}{T}}$, where $T_{0}$ and $T$ are the desired and the current temperatures, respectively. 
Figure 5.1 Divergence of trajectories in molecular dynamics for $\mathrm{Fe}_{3} \mathrm{Al}$ at $\mathrm{T}=293 \mathrm{~K}$

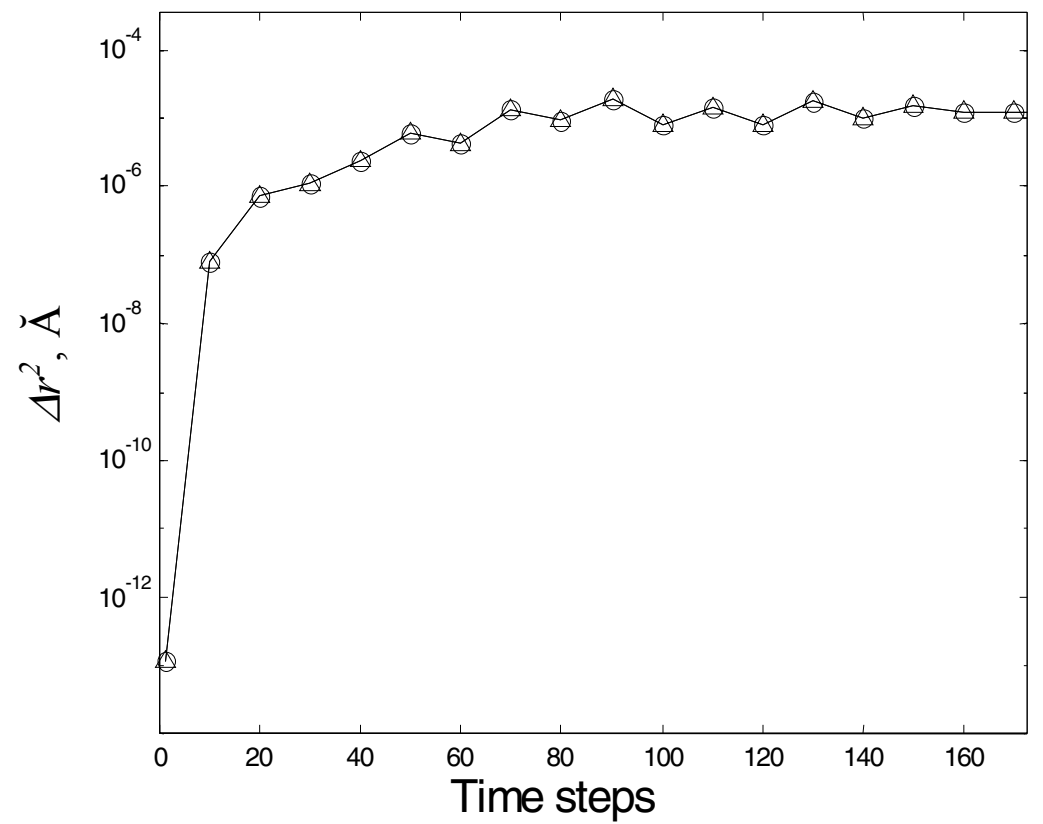

Figure 5.2 Energy conservation during molecular dynamics for $\mathrm{Fe}_{3} \mathrm{Al}$ at $\mathrm{T}=293 \mathrm{~K}$ The initial configurations of atoms are the same as in Fig.5.1. Two energy-curves do coincide.

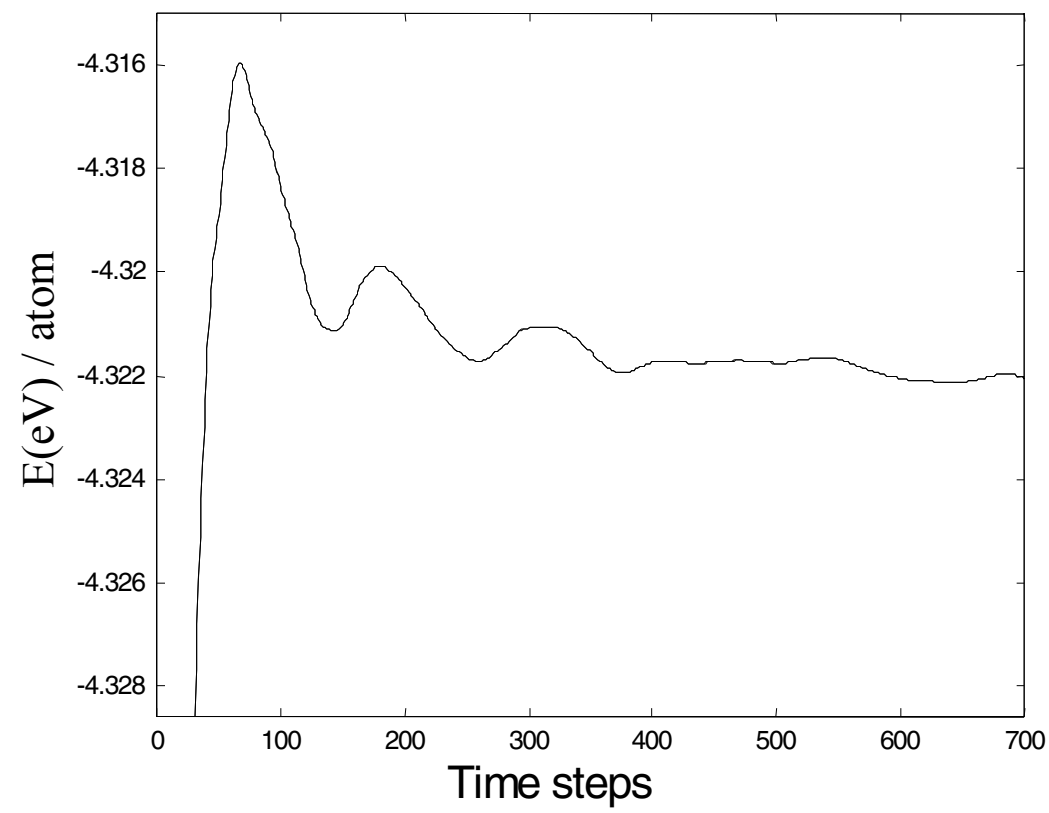


This method is identical to solving a system of perturbed Newtonian equations of motion:

$$
\begin{aligned}
& \dot{r}_{i}=p_{i} / m_{i} \\
& \dot{p}_{i}=f_{i}-\xi(r, p) p_{i},
\end{aligned}
$$

the quantity $\xi(r, p)$ guaranties that temperature $T$ remains constant. It is chosen so as perturb as little as possible the classical equations of motion and satisfies the following relationship:

$$
\xi(r, p)=\frac{\sum_{i} p_{i} f_{i}}{\sum_{i}\left|p_{i}\right|^{2}} .
$$

To control the pressure during the simulation we used an extended system method proposed by Andersen [10]. It is based on imitating the action of a piston with some mass assigned to it. The kinetic and potential energies of the piston depend on the volume of the system and the atomic positions can be represented in terms of scaled variables:

$$
r_{i}=V^{1 / 3} s_{i}, v_{i}=V^{1 / 3} \dot{s}_{i}
$$

Then the equations of motion can be obtained from the Lagrangian that includes both energies of atomic system and those of the piston:

$$
\begin{aligned}
\ddot{s}_{i} & =f_{i} /\left(m V^{1 / 3}\right)-\frac{2}{3} s_{i} \dot{V} / V, \\
\ddot{V} & =\left(P^{1}-P\right) / Q
\end{aligned}
$$

The equations are solved using Gear predictor-corrector algorithm (Equations 5.3, 5.5) treating volume of the system as an external parameter. 


\subsubsection{Many-body atomistic potentials for iron-aluminides with the addition of transition metals}

There are a lot of different ways to parameterize the total energy of a crystal. To achieve computational efficiency required in MD simulations, we have chosen ab initio based manybody atomistic potentials. In this method the interaction between the atoms is represented as a functional of the atomic configuration. The choice of this form depends on the physical properties of a crystal under consideration. In iron-aluminides the aluminum sp-electrons tend to get highly hybridized with the iron d-electrons that makes the bonding between the atoms to have both covalent and metallic features. Thus, we divide the total energy into the following three parts: many-body interaction energy, pair-potential and three-body interaction energy:

$$
E=\sum_{i} e_{i}\left(\rho_{i},\left\{p_{1}\right\}\right)+\sum_{i \neq j} V_{2}\left(r_{i j},\left\{p_{2}\right\}\right)+\sum_{i, j \neq i, k \neq i} V_{3}\left(r_{i j}, r_{i k},\left\{p_{3}\right\}\right)
$$

where $\left\{\mathrm{p}_{1}\right\},\left\{\mathrm{p}_{2}\right\}$ and $\left\{\mathrm{p}_{3}\right\}$ are the parameters to be calculated by fitting.

The first term on the right-hand side of the equation (5.12) is the energy $\mathrm{e}_{\mathrm{si}}$ of the ith atom as a function of the atomic density $\rho_{i}$ and represents the many-body interaction that is typical for metallic crystals. In the metals the valence electrons are completely separated from their ion cores and form a merely uniform gas. Thus, each atom in a solid is interacting with the background electronic charge that is represented by the atomic density. This physical fact gave rise to Embedded Atom Methods (EAM) [11,12,13] and our many-body atomistic potentials belong to this big group.

The atomic density of a pure metal with a given volume is defined as a relationship of 
this volume to the volume of the equilibrium and it can be written as follows:

$$
\rho_{i}=\frac{\omega_{e i}}{\omega_{i}}=f_{i}+\sum_{j} \xi_{j}\left(r_{i j}\right)
$$

Here, on the right-hand side the summation is carried over all neighbor atoms of the $i$-th atom with the distance given by $r_{i j}$. Hence, the density function is a dimensionless variable and for a pure metal in a ground state it is equal to one. For a multi-component compound, the density becomes a local variable depending on the number of the nearest neighbors and their distances from the atom $i$. To determine the function $\rho_{i}$, we choose a reference lattice structure, then uniformly expand and contract the crystal lattice. The equilibrium volume is obtained from the first principle calculations. The following form of the function $\xi(r)$ is found to be the simplest and the most flexible for fitting atomic densities of $b c c, f c c$ and $h c p$ structures simultaneously:

$$
\xi(r)=q r^{k}[\exp (-\zeta r)-y \exp (-\eta r)]
$$

Together with $f_{i}$ there are six parameters for the density function for a given type of atoms. They can be determined from the fitting. We fit our parameters exclusively from the data obtained from FP-LMTO calculations. One of the main error sources in FP-LMTO method is due to the limited sampling of the Brillouin zone while integrating the energy eigenvalues. In order to minimize this systematic error, we considered the structures with the same symmetry for all pure metals and their compounds. Thus, we have chosen $b c c$ structure for the reference, since the iron-aluminides structures, namely $\mathrm{DO}_{3}$ and $\mathrm{B}_{2}$, are $b c c$ based.

In practice, the values of the atomic density parameters depend on the cutoff distance of $\rho\left(r_{i}\right)$. To minimize this effect, the cutoff distance has to be situated in between the neighboring lattice shells. Unfortunately, the lattice constants of $\mathrm{FeAl}$ and $\mathrm{Fe}_{3} \mathrm{Al}$ are very different from the 
lattice constants of pure Mo and V metals. Therefore, we have chosen cutoff distance to be dependent on the type of the atoms. The cutoff function has the same distance dependence:

$$
F_{\text {cutoff }}\left(r_{i j}\right)=(1-x)^{3}\left(1+3 x+6 x^{2}\right), x=\frac{r_{i j}-r_{b}}{r_{c}-r_{b}} \text {, }
$$

where $r_{c}$ is the cutoff distance and $r_{b}$ is the point, where cutoff function gets connected with the density function. Such a form of the cutoff function guarantees that the first and the second-order derivatives of $F_{\text {cutof }}\left(r_{i j}\right)$ are equal to zero at $r_{b}$ and $r_{c}$, respectively.

The values of the cutoff distances together with the obtained density parameters are listed in the Table 5.1 and Table 5.2 correspondingly. The density parameters were tested with respect to the small disturbance of the cutoff distance.

Table 5.1 Cutoff distances in A for different atomic types.

\begin{tabular}{|c|c|c|c|c|}
\hline & $\mathrm{Al}$ & $\mathrm{Fe}$ & $\mathrm{V}$ & Mo \\
\hline$r_{b}$ & 5.10 & 5.10 & 5.30 & 5.70 \\
\hline$r_{c}$ & 5.20 & 5.20 & 5.40 & 5.80 \\
\hline
\end{tabular}

Table 5.2 Atomic density parameters found from the fitting of the relationship given by (5.12). The interatomic distances are scaled by $r_{e}$, were $r_{e}$ is the bond length of the pure metals. The parameters are dimensionless.

\begin{tabular}{|c|c|c|c|c|c|c|c|}
\hline Type of Atom & $r_{e}, A$ & $f$ & $Q$ & $\zeta$ & $K$ & $Y$ & $\eta$ \\
\hline $\mathrm{Al}$ & 2.75 & 0.4034 & 55.85 & 2.826 & 0.303 & 0.967 & 2.828 \\
\hline $\mathrm{Fe}$ & 2.337 & 0.2264 & 53.76 & 2.844 & 0.953 & 0.969 & 2.830 \\
\hline $\mathrm{V}$ & 2.52 & 0.2358 & 54.13 & 2.844 & 0.908 & 0.969 & 2.830 \\
\hline $\mathrm{Mo}$ & 2.704 & 0.2443 & 54.33 & 2.844 & 0.853 & 0.969 & 2.830 \\
\hline
\end{tabular}


The functional form of the density-dependent energy in (5.12) should be rich enough to describe all possible deformations of the system and therefore, we define this functional implicitly through its relationship to the uniform expansion energy curve $E(\omega)$ :

$$
N \cdot e_{s i}\left(\rho_{i},\left\{p_{1}\right\}\right)=E(\omega)-\sum_{i \neq j} V_{2}\left(r_{i j},\left\{p_{2}\right\}\right)-\sum_{i, j \neq i, k \neq i} V_{3}\left(r_{i j}, r_{i k},\left\{p_{3}\right\}\right)
$$

where $N$ is the total number of the atoms, $\omega$ is the volume of the crystal. Since during the uniform expansion the symmetry of the crystal is completely preserved, the distances $r_{i j}$ between the atoms are the functions of the atomic density. The first term in (5.15) is the total energy variation for the volume expansion obtained from the first-principle calculations. We parameterize it in the following form:

$E(\omega) / N=e_{0}-2 e_{1} \exp \left(-\alpha \frac{\omega_{e}}{\omega}\right)+e_{2} \exp \left(-2 \alpha \frac{\omega_{e}}{\omega}\right)$

In Table 5.3 we listed the calculated values of the parameters in (5.17). The pair-potentials in (5.12) we choose to have the following form:

$$
\phi(r)=\phi_{0}\left[1+s_{1}\left(r / r_{0}-1\right)\right] \exp \left[-s_{2}\left(r / r_{0}-1\right)\right]
$$

For each pair of interacting atoms there are four parameters that depend on the atomic types.

Table 5.3 Potential parameters for lattice expansion energy curve (5.17), for bcc structure.

\begin{tabular}{|l|l|l|l|l|l|}
\hline Atom & $e_{0}(\mathrm{eV})$ & $e_{1}(\mathrm{eV})$ & $E_{2}(\mathrm{eV})$ & $\alpha$ & $\omega_{e}\left(A^{3}\right)$ \\
\hline $\mathrm{Al}$ & -2.117 & 7.807 & 54.135 & 1.959 & 32.019 \\
\hline $\mathrm{Fe}$ & -0.822 & 19.177 & 98.666 & 1.639 & 19.651 \\
\hline $\mathrm{V}$ & -1.445 & 15.285 & 67.558 & 1.482 & 24.638 \\
\hline $\mathrm{Mo}$ & -1.544 & 27.565 & 143.90 & 1.621 & 30.439 \\
\hline
\end{tabular}


For the same types of atoms the cutoff distance of the pair-potential is chosen the same as for the density function of atoms of that type. The interaction between the atoms of different types takes place only in the compounds, therefore, the cutoff distance was chosen to be in between fifth and sixth neighboring shells that results in taking into account up to 58 next-neighbors in $\mathrm{B}_{2}$ and $\mathrm{DO}_{3}$ structures. This cutoff distance is equal to $5.20 \mathrm{~A}$ and the distance of the cutoff function connection is 5.10A. The parameters for pair-interaction are given in Table 5.4.

One can see that the density-dependent energy and the pair-potentials have a spherical symmetry. However, in covalent crystals the strength of the interatomic interaction depends strongly on the direction. To describe the angular dependence of the bonding we use the threebody interaction.

Table 5.4 Pair-potential parameters for expression given by (5.18), found by fitting FP-LMTO total energies for the compound with the lattice under deformations.

\begin{tabular}{|c|c|c|c|c|}
\hline Atom-atom & $\phi_{0}(\mathrm{eV})$ & $r_{0}(\mathrm{~A})$ & $s_{1}$ & $s_{2}$ \\
\hline $\mathrm{Al}-\mathrm{Al}$ & -0.13113 & 2.75 & 22.36 & 6.425 \\
\hline $\mathrm{Fe}-\mathrm{Fe}$ & -0.5113 & 2.41 & 6.506 & 5.799 \\
\hline $\mathrm{Fe}-\mathrm{Al}$ & -0.4024 & 2.45 & 8.620 & 6.300 \\
\hline $\mathrm{V}-\mathrm{V}$ & -0.0492 & 2.52 & 8.821 & 6.300 \\
\hline $\mathrm{V}-\mathrm{Al}$ & -0.9630 & 2.80 & 7.656 & 4.461 \\
\hline $\mathrm{V}-\mathrm{Fe}$ & -0.1178 & 2.45 & 4.770 & 4.245 \\
\hline $\mathrm{Mo-Mo}$ & -0.1537 & 2.704 & 10.163 & 8.109 \\
\hline $\mathrm{Mo}-\mathrm{Al}$ & -0.6157 & 2.800 & 8.7040 & 3.043 \\
\hline $\mathrm{Mo}-\mathrm{Fe}$ & -0.0800 & 2.450 & 5.5092 & 5.100 \\
\hline
\end{tabular}


Three-body interaction is given by:

$$
V_{3}=\frac{1}{2} \sum_{j \neq i, k \neq i} w\left(r_{i j}\right) w\left(r_{i k}\right)\left(\cos ^{2} \Theta_{i j k}-\frac{1}{3}\right), w\left(r_{i j}\right)=g_{i j} \exp \left(-\frac{\gamma_{i j}}{r_{c}-r_{i j}}\right)
$$

where $r_{i j}, r_{i k}$, are the radius vectors of the atoms $j$ and $k$ drawn from the $i t h$ atom as a center, $\Theta_{i j k}$ is the angle between these radius vectors. The form of the radius-dependent part $w_{i j}(r)$ provides flexibility necessary for the fitting. This function can have a positive curvature or a negative one depending on the value of the parameter $\gamma_{i j}$. The angular-dependent part of the function $h\left(\Theta_{i j k}\right)$ is taken from the work of Besson et al [1]. It provides the convenience that three-body interaction is equal to zero in undistorted $b c c$ based and $f c c$ structures. Thus, $V_{3}$ cancels out in expression (5.15) for the uniformly expanded structures that saves some of the computational time. We have chosen $3.50 \mathrm{~A}$ to be a cutoff distance for three-body interaction energy. Three-body interaction parameters are listed in Table 5.4.

While the parameters of the density-dependent part were found from the uniform expansion of pure materials, the pair-potential parameters and three-body interaction energy were found from the fitting the total energies of the iron-aluminides. In case of pure ironaluminides there are 18 parameters to be fitted, but when Mo or $\mathrm{V}$ additives are present in the compound this number increases to 36 . To make our potentials working far from the equilibrium, we fitted the total energies of a crystal obtained from the FP-LMTO calculations under various deformations.

Since we need these atomistic potentials to study the thermal properties of the ironaluminides, it is important for us to have a fair description of the elastic properties of these compounds as well as the information the anharmonicity rate of the lattice phonons. This explains our choice of the crystal deformations used in the fitting procedure that are: uniform 
Table 5.5 Three-body interaction parameters for use in the expression given by (5.19) found by fitting FP-LMTO total energies for distorted lattices.

\begin{tabular}{|c|c|c|c|c|c|c|c|c|c|}
\hline & Al-Al & Fe-Fe & Fe-Al & V-V & V-Al & V-Fe & Mo-Mo & Mo-Al & Mo-Fe \\
\hline$g_{i j}$ & 0.6255 & 0.4943 & 1.8306 & 1.165 & 1.831 & 0.494 & 1.451 & 1.831 & 0.494 \\
\hline$\gamma_{i j}$ & 0.1661 & 0.0629 & 2.1530 & 1.298 & 2.153 & 0.063 & 0.6949 & 2.153 & 0.063 \\
\hline
\end{tabular}

expansion, volume conserving orthorombic strain and volume conserving monoclinic strain. These deformations provide a convenient way of calculating the elastic constants, as it was described in Chapter 4.2. To capture the anharmonic behavior of the phonons we performed these deformations at different volumes that provided us the information of how the elasticity of a crystal is changing with the expansion. The parameters were fitted, in a least-square sense, by anti-gradient decent method [14]. The comparison between obtained total energies and results of the fitting procedure are presented in Figures $5.3(a, b)$. In Figure 5.3(a) the first 120 deformations correspond to $\mathrm{FeAl}$ compound and the rest of them are related to deformations of $\mathrm{Fe}_{3} \mathrm{Al}$. The notation $\mathrm{U}^{*}$ in the bottom part of the graph indicates the uniform expansion of the compound, the notation $\mathrm{c} 11 \mathrm{c} 12 *$ is used for the orthorhombic strain and by $\mathrm{c} 44^{*}$ we denoted the monoclinic strain. Since the two energy curves completely coincided in Figure 5.3(a), we showed a zoomed part of the curve in Figure 5.3(b). 


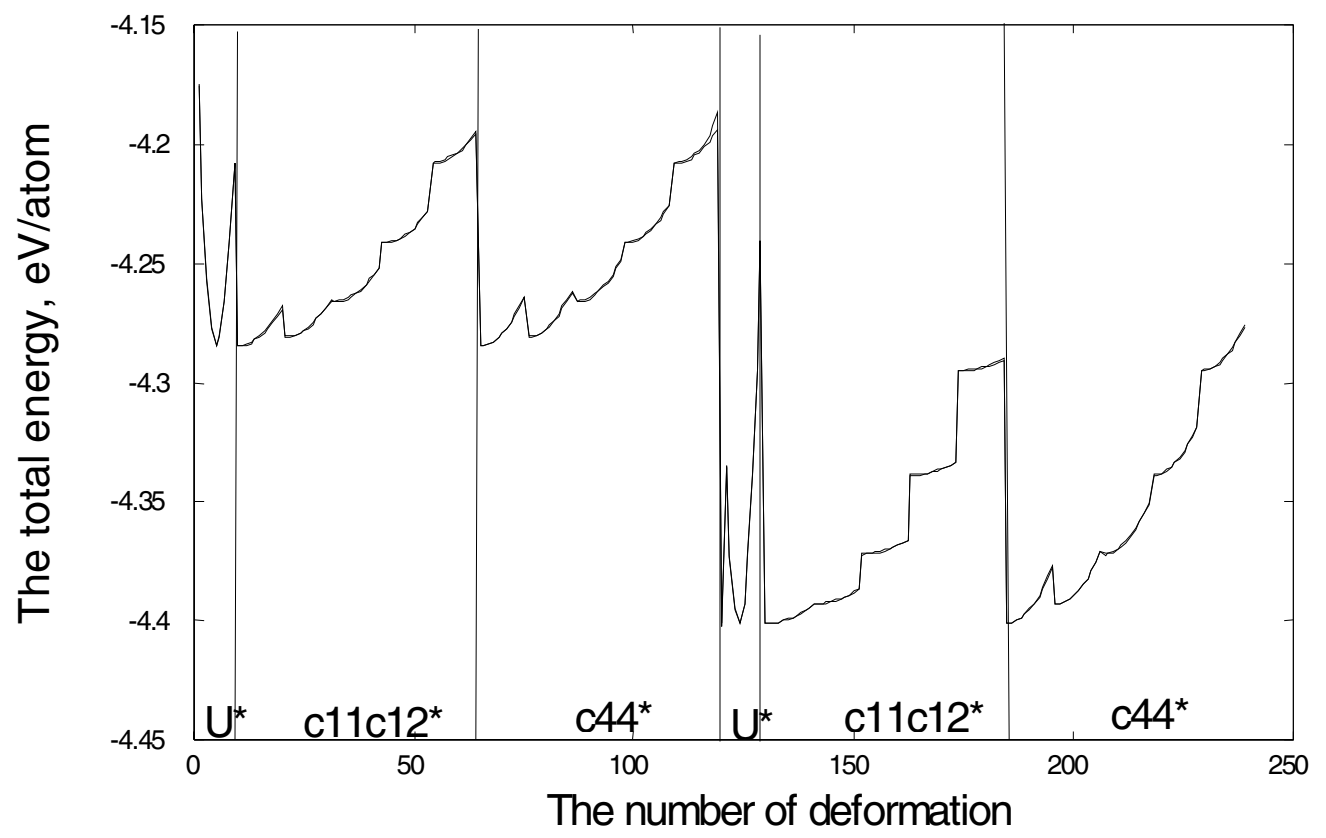

Figure 5.3 (a) The results of the fitting procedure.

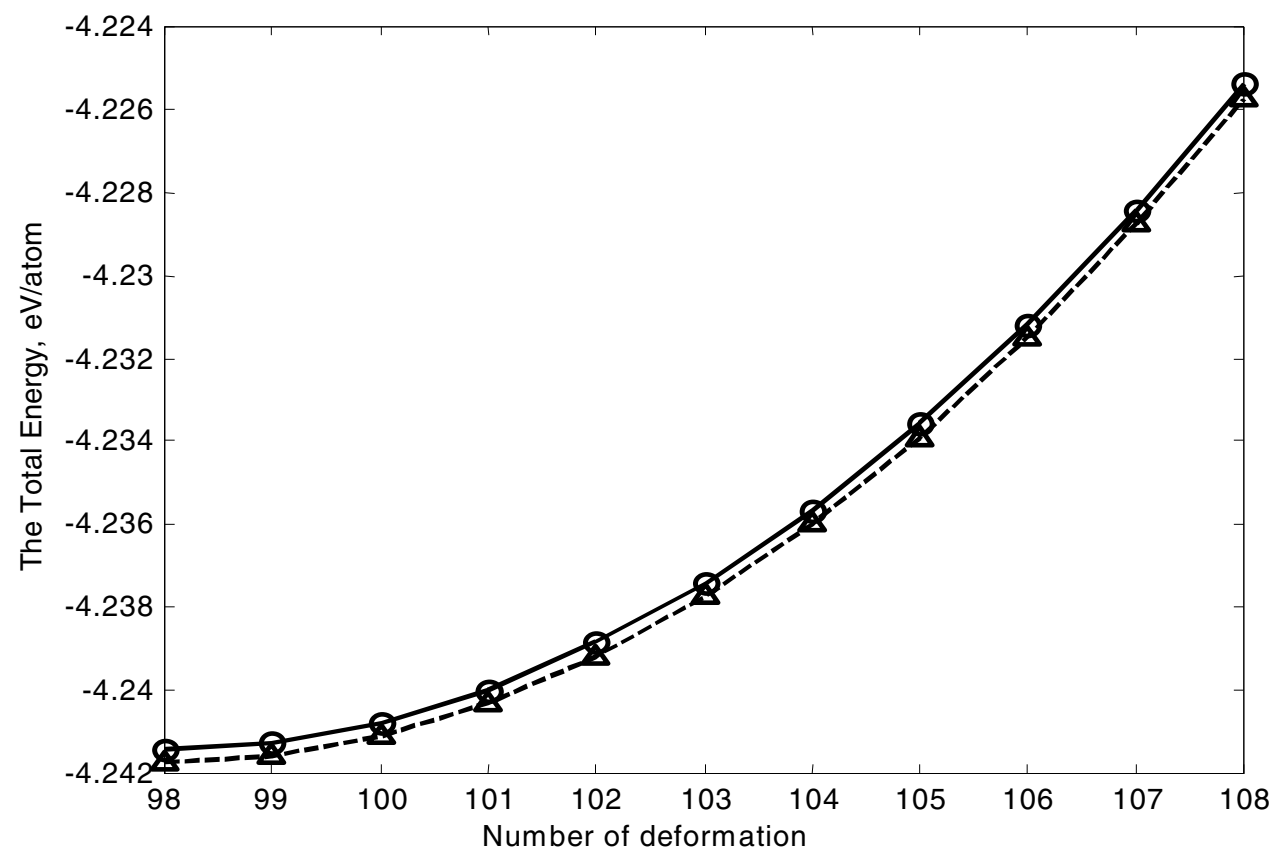

Figure 5.3 (b) The results of the fitting procedure. The circles represent the total energy of FeAl and $\mathrm{Fe}_{3} \mathrm{Al}$ calculated by FP-LMTO method. The triangles show the results of many-body atomistic potentials. 
The most difficult part of the fitting was to obtain the pair-potential parameters that guarantee the total energy of a system to have an absolute minimum when the lattice is undistorted. The necessary condition of parameters to be stable is that the density-dependent energy is attractive and the pair-potentials are repulsive when distance between the atoms is shorter than the bonding length. If the condition is not satisfied the atoms will find such a configuration when some of the distances become extremely short while the others increase significantly, hence conserving the atomic density. In such a situation the increase of the densitydependent part will not compensate for the decrease of the pair-interaction energy and therefore, the total energy of the distorted lattice will be smaller than that in the equilibrium. To make sure that the fitted parameters are stable, it is necessary to look at the location of minimum of the pair potentials. Obviously, the minimum of the function (5.18) satisfies the condition:

$$
r-r_{0}=\frac{\left(s_{1}-s_{2}\right)}{s_{1} s_{2}} r_{0} .
$$

Therefore, we initiated the parameters fitting with fixed $r_{0}$ equal to the bond length between the atoms at the reference structure and calculated $s_{1}, s_{2}$ that were at first set to satisfy $s_{1} \sim s_{2}$. After minimizing the total energy mean-square deviation from FP-LMTO calculations, the parameters $r_{0}$ were also relaxed. The obtained pair-potentials and 3-body interaction distance dependence is shown in Figures 5.4 and 5.5, respectively. 


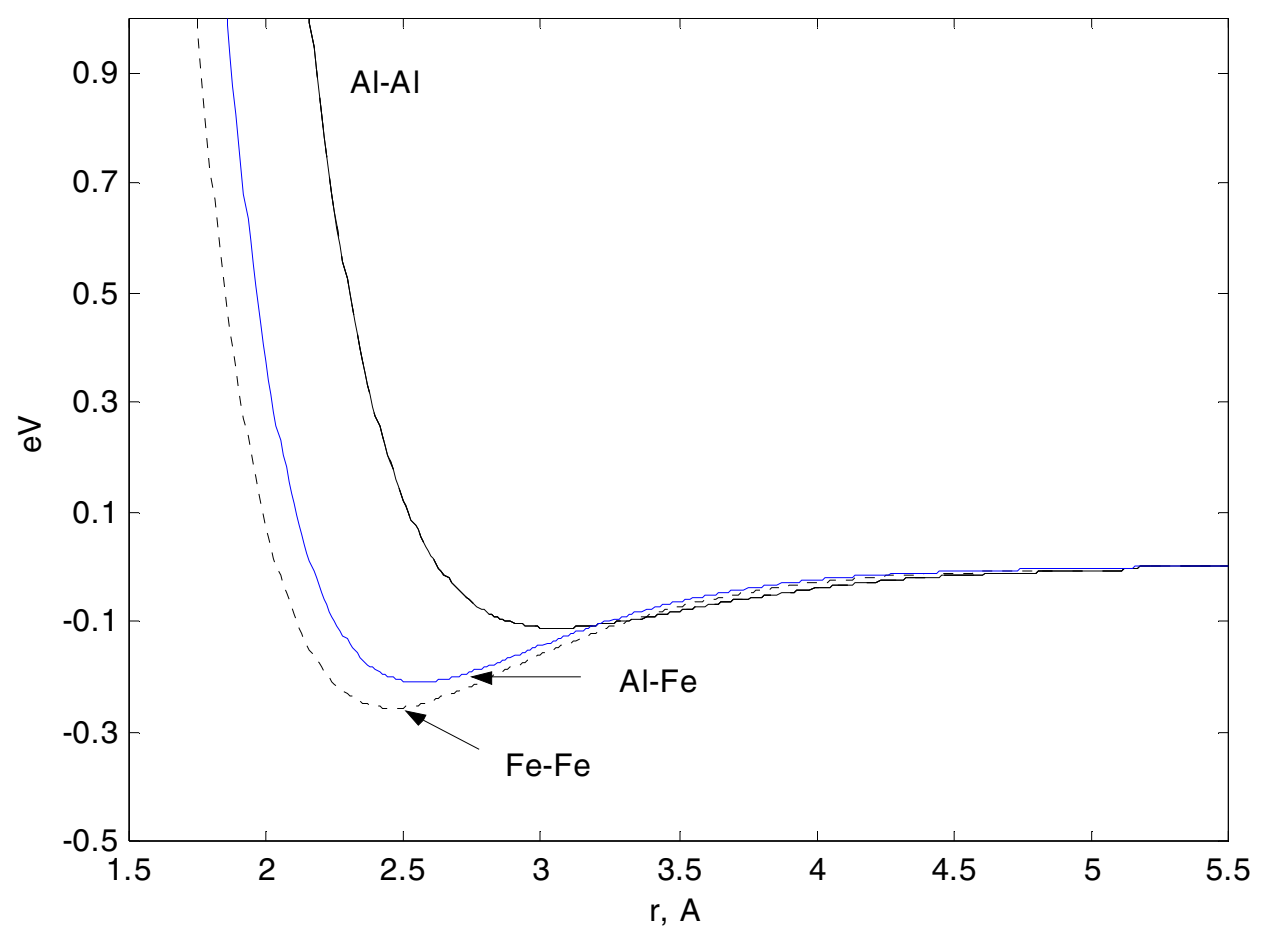

Figure 5.4 Distance-dependence of pair-potentials given by 5.17

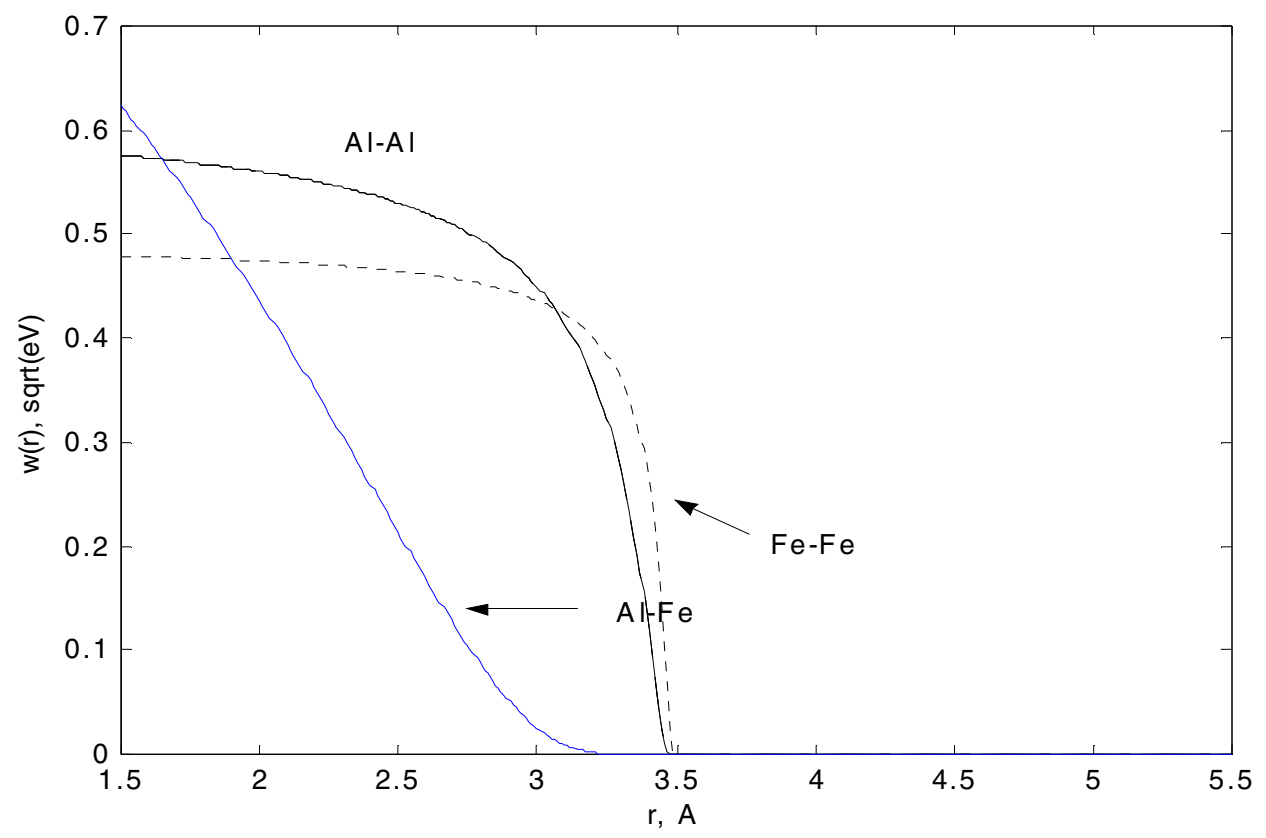

Figure 5.5 Distance-dependence of 3-body term given by 5.19 


\subsection{Results}

\subsubsection{Pure FeAl and $\mathrm{Fe}_{3} \mathrm{Al}$}

a) Stability of the structure. The first test for obtained atomistic potentials was to examine the relative stability of several lattice structures of iron-aluminides. For FeAl, we compared the total energies of $\mathrm{B}_{2}$ and $\mathrm{B}_{32}$ crystallographic lattice structures. For $\mathrm{Fe}_{3} \mathrm{Al}, \mathrm{DO}_{3}$ and $\mathrm{L}_{1}$ structures were considered. The primitive vectors together with the basis coordinates of these structures are given in the Table 5.6. The results of calculation are compared to ab initio calculations $[15,16]$ and semi-empirical modified embedded method of Besson et al ${ }^{1}$ in Table 5.7.

Table 5.6 Lattice vectors and basis of the selected lattice structures. The vectors are represented in Cartesian coordinates. The lattice constant is denoted by letter $\mathbf{a}$.

\begin{tabular}{|c|c|c|}
\hline & Primitive vectors & Basis vectors \\
\hline $\mathrm{B}_{2}$ & $\begin{array}{l}\mathbf{A}_{1}=\mathrm{a} \mathbf{X} \\
\mathbf{A}_{2}=\mathrm{a} \mathbf{Y} \\
\mathbf{A}_{3}=\mathrm{a} \mathbf{Z}\end{array}$ & $\begin{array}{l}\mathbf{B}_{1}=0 \\
\mathbf{B}_{3}=+1 / 2 \text { a } \mathbf{X}+1 / 2 \text { a } \mathbf{Y}+1 / 2 \text { a } \mathbf{Z}\end{array}$ \\
\hline $\mathrm{B}_{32}$ & $\begin{array}{l}\mathbf{A}_{1}=1 / 2 \text { a } \mathbf{Y}+1 / 2 \text { a } \mathbf{Z} \\
\mathbf{A}_{2}=1 / 2 \text { a } \mathbf{X}+1 / 2 \text { a } \mathbf{Z} \\
\mathbf{A}_{3}=1 / 2 \text { a } \mathbf{X}+1 / 2 \text { a } \mathbf{Y}\end{array}$ & $\begin{array}{l}\mathbf{B}_{3}=+1 / 8 \text { a } \mathbf{X}+1 / 8 \text { a } \mathbf{Y}+1 / 8 \text { a } \mathbf{Z}(\mathrm{Al}) \\
\mathbf{B}_{3}=-1 / 8 \text { a } \mathbf{X}-1 / 8 \text { a } \mathbf{Y}-1 / 8 \text { a } \mathbf{Z}(\mathrm{Al}) \\
\mathbf{B}_{3}=+3 / 8 \text { a } \mathbf{X}+3 / 8 \text { a } \mathbf{Y}+3 / 8 \text { a } \mathbf{Z}(\mathrm{Fe}) \\
\mathbf{B}_{3}=-3 / 8 \text { a } \mathbf{X}-3 / 8 \text { a } \mathbf{Y}-3 / 8 \text { a } \mathbf{Z}\end{array}$ \\
\hline $\mathrm{DO}_{3}$ & $\begin{array}{l}\mathbf{A}_{1}=\mathrm{a} \mathbf{Y}+\mathrm{a} \mathbf{Z} \\
\mathbf{A}_{2}=\mathrm{a} \mathbf{X}+\mathrm{a} \mathbf{Z} \\
\mathbf{A}_{3}=\mathrm{a} \mathbf{X}+\mathrm{a} \mathbf{Y}\end{array}$ & $\begin{array}{l}\mathbf{B}_{1}=0 \\
\mathbf{B}_{2}=\mathrm{a} \mathbf{X} \\
\mathbf{B}_{3}=+1 / 2 \mathrm{a} \mathbf{X}+1 / 2 \mathrm{a} \mathbf{Y}+1 / 2 \mathrm{a} \mathbf{Z} \\
\mathbf{B}_{4}=-1 / 2 \mathrm{a} \mathbf{X}-1 / 2 \mathrm{a} \mathbf{Y}-1 / 2 \mathrm{a} \mathbf{Z}\end{array}$ \\
\hline $\mathrm{L} 2_{1}$ & $\begin{array}{l}\mathbf{A}_{1}=\mathrm{a} \mathbf{X} \\
\mathbf{A}_{2}=\mathrm{a} \mathbf{Y} \\
\mathbf{A}_{3}=\mathrm{a} \mathbf{Z}\end{array}$ & $\begin{array}{l}\mathbf{B}_{1}=0 \\
\mathbf{B}_{2}=+1 / 2 \text { a } \mathbf{X}+1 / 2 \text { a } \mathbf{Y} \\
\mathbf{B}_{3}=+1 / 2 \text { a } \mathbf{X}+1 / 2 \text { a } \mathbf{Z} \\
\mathbf{B}_{4}=+1 / 2 \text { a } \mathbf{Y}+1 / 2 \text { a } \mathbf{Z}\end{array}$ \\
\hline
\end{tabular}


Table 5.7 Computed structural data for $\mathrm{FeAl}$ and $\mathrm{Fe}_{3} \mathrm{Al}$ within many-body atomistic potentials, ab initio calculations and semi-empirical method of Besson et al .

\begin{tabular}{|c|c|c|c|c|c|c|c|}
\hline & & \multicolumn{2}{|c|}{$a_{0}, A$} & \multirow[t]{2}{*}{$E_{0}$} & \multirow{2}{*}{$\begin{array}{l}\Delta E, \\
\text { Calc. }\end{array}$} & \multirow{2}{*}{$\begin{array}{c}\Delta E, \\
a b \text { initio }\end{array}$} & \multirow{2}{*}{$\begin{array}{c}\Delta E, \text { Besson } \\
\text { et } a l^{\mathrm{d}}\end{array}$} \\
\hline & & Calc. & $\operatorname{Exp} .^{\mathrm{a}}$ & & & & \\
\hline \multirow[t]{2}{*}{$\mathrm{FeAl}$} & $\mathrm{B}_{2}$ & 2.805 & 2.903 & -4.25 & \multirow[t]{2}{*}{0.48} & \multirow[t]{2}{*}{$0.10^{b}$} & \multirow[t]{2}{*}{0.05} \\
\hline & $\mathrm{B}_{32}$ & & & -3.77 & & & \\
\hline \multirow[t]{2}{*}{$\mathrm{Fe}_{3} \mathrm{Al}$} & $\mathrm{DO}_{3}$ & 5.505 & 5.780 & -4.40 & \multirow[t]{2}{*}{0.09} & \multirow[t]{2}{*}{$0.071^{\mathrm{c}}$} & \multirow[t]{2}{*}{0.35} \\
\hline & $\mathrm{Ll}_{2}$ & & & -4.31 & & & \\
\hline \multicolumn{8}{|c|}{${ }^{\mathrm{a}}$ Reference 15} \\
\hline \multicolumn{8}{|c|}{${ }^{\mathrm{b}}$ Reference 16} \\
\hline \multicolumn{8}{|c|}{${ }^{\mathrm{c}}$ Reference 17} \\
\hline \multicolumn{8}{|c|}{${ }^{\mathrm{d}}$ Reference 1} \\
\hline
\end{tabular}

For FeAl, the total energy of $\mathrm{B}_{2}$ structure is lower than the energy of $\mathrm{B}_{32}$ indicating that $\mathrm{B}_{2}$ is the ground-state structure of $\mathrm{FeAl}$. For $\mathrm{Fe}_{3} \mathrm{Al}$ our potentials predict that it has $\mathrm{DO}_{3}$ structure in the ground state. These two results are in a good agreement with ab initio calculations and experiment. However, the relative energies of the different structures are not exactly the same with the results of ab initio calculations. There is a general difficulty in semi-empirical methods to obtain atomistic potentials that are utterly transferable from one lattice structure to another. However, calculating the thermal expansion one is considering the system to be close to the equilibrium. Therefore, the most important issue about the obtained atomistic potentials is the correctly determined ground-state structure for both $\mathrm{FeAl}$ and $\mathrm{Fe}_{3} \mathrm{Al}$. 
b) Point defects We calculated the point defect energies in FeAl and $\mathrm{Fe}_{3} \mathrm{Al}$ using the usual definition:

$$
E^{f}=T E_{\text {defect }}+\mu_{\text {defect }}-T E_{\text {perfect }}
$$

where $T E_{\text {defect }}$ is the total energy of a supercell with a defect; $T E_{\text {perfect }}$ is the total energy of a corresponding perfect supecell; $\mu_{\text {defect }}$ is the chemical potential due to substituting original atom by the impurity, which is defined as the bulk energy per atom of elemental metal.

The calculated energies of defects of $\mathrm{FeAl}$ and $\mathrm{Fe}_{3} \mathrm{Al}$ are presented. in Tables 5.8 and 5.9, respectively The results of $a b$ initio calculations and available experimental data are provided for comparison.

Table 5.8 Point defect energies $(\mathrm{eV})$ for $\mathrm{B}_{2}$ FeAl. The subscript $v$ stands for vacancy, the subscript $a$ is used for anti-site defect.

\begin{tabular}{|c|c|c|c|c|}
\hline & $E_{\text {relaxation }}$ & $E_{\text {formation }}$ & $E_{\text {formation, }}$ ab initio $^{a}$ & $E_{\text {formation, }}$ experiment $^{b}$ \\
\hline$E_{v}{ }^{A l}$ & 0.85 & 4.70 & 4.00 & \\
\hline$E_{v}{ }^{F e}$ & 0.60 & 3.42 & 0.97 & 0.70 \\
\hline$E_{a}{ }^{\mathrm{Al}>\mathrm{Fe}}$ & 1.15 & 2.43 & 1.04 & \\
\hline$E_{a}{ }^{\mathrm{Fe}>A l}$ & 0.00 & 2.49 & 0.95 & \\
\hline
\end{tabular}

\footnotetext{
${ }^{a}$ Reference 18

${ }^{b}$ Reference 19
} 
Table 5. 9 Point defect energies (eV) for $\mathrm{DO}_{3} \mathrm{Fe}_{3} \mathrm{Al}$. The subscript $v$ stands for vacancy, the subscript $a$ is used for anti-site defect.

\begin{tabular}{|c|c|c|c|c|}
\hline & $E_{\text {relaxation }}$ & $E_{\text {relaxation, }}$ ab initio ${ }^{a}$ & $E_{\text {formation }}$ & $E_{\text {formation }}, a$ initio $^{a}$ \\
\hline$E_{v}{ }^{A l}$ & 1.00 & 0.60 & 4.01 & 5.80 \\
\hline$E_{v}{ }^{F e I}$ & 1.28 & 0.04 & 3.49 & 4.03 \\
\hline$E_{v}^{\text {Fell }}$ & 1.54 & 0.65 & 3.39 & 2.35 \\
\hline$E_{a}^{F e->A l}$ & 0.07 & 0.00 & 2.56 & 4.90 \\
\hline$E_{a}{ }^{A l->F e l}$ & 0.26 & 0.07 & 1.07 & 0.18 \\
\hline$E_{a}^{A l->F e l l}$ & 0.89 & 0.19 & 2.21 & 1.91 \\
\hline
\end{tabular}

\section{${ }^{a}$ Reference 20}

Before analyzing the obtained results, we have to mention that the two models used for defects simulation in many-body atomistic potentials and ab initio methods considerably differ from each other. While in ab initio methods the atomic relaxation around the defect is allowed only within the prescribed symmetry, many-body atomistic potentials incorporated into the molecular dynamics take into account all possible atomic degrees of freedom. Therefore, the relaxation energies obtained by many-body atomistic potentials are generally higher than those calculated from the first-principles and therefore, the defect formation energies are lower.

The defect formation energies for FeAl in Table 5.8 appear to be quite different from the results of ab initio calculations. The $a b$ initio results [18] were obtained by mixed-basis pseudopotential method that is different from FP-LMTO method used in the atomistic potential fitting and what is more essential, using a different definition of the chemical potential in (5.20). However, the disagreement appears to be only in the absolute values of defect formation energies. The relative formation energies, which determine actual defects concentration, are in a 
good agreement with $a b$ initio calculations for both $\mathrm{FeAl}$ and $\mathrm{Fe}_{3} \mathrm{Al}$. Here we briefly discuss the physical meaning of the defect formation energies.

The smallest relaxation energy was obtained for $\mathrm{Fe}$ - $>\mathrm{Al}$ anti-site defect for both $\mathrm{FeAl}$ and $\mathrm{Fe}_{3} \mathrm{Al}$. Since these compounds get stabilized at the lattice constant insignificantly larger than the lattice constant of pure iron, the atomic displacements caused by Fe placed at Al site are negligibly small. When placing $\mathrm{Al}$ atom at $\mathrm{Fe}$ site, the relaxation energies significantly increase. The strongest relaxation $\sim 1.54 \mathrm{eV}$ corresponds to FeII vacancy in $\mathrm{Fe}_{3} \mathrm{Al}$ compound.

The relationship between the defect formation energies in $\mathrm{FeAl}$ is found to be quite similar to the one in $\mathrm{Fe}_{3} \mathrm{Al}$. In $\mathrm{FeAl}$ and $\mathrm{Fe}_{3} \mathrm{Al}$ the highest defect formation energy corresponds to $\mathrm{Al}$ vacancy that is in a good agreement with ab initio calculations. In both aluminides the obtained monovacancy formation energies are higher than the formation energies of anti-site defects. Judging from the values of formation energies, $A l->F e$ anti-site defect appears to be the most common in $\mathrm{FeAl}$ and $\mathrm{Fe}_{3} \mathrm{Al}$ compounds. This is not surprising, since substitution of all FeI sites in $\mathrm{DO}_{3}$ structure by $\mathrm{Al}$ atoms results in a formation of $\mathrm{FeAl}$ found in $\mathrm{B}_{2}$ structure, and such a second-order transition occurs in practice at $830 \mathrm{~K}$. In addition, the low anti-site defect formation energies explain FeAl normal $<111>$ slip behavior that was observed experimentally [21]. In $\mathrm{B}_{2}$ structure $<111>$ partial slip necessarily brings together $\mathrm{Fe}$ and $\mathrm{Al}$ atoms that can easily interchange their lattice sites, while disturbing the lattice symmetry.

Using the grand canonical formalism on a rigid lattice, we estimated the defect concentrations in $\mathrm{FeAl}$ and $\mathrm{Fe}_{3} \mathrm{Al}$ at their perfect stochiometry. The obtained concentrations are found to be extremely lower than the one determined experimentally $[22,23,24]$. However, in our calculations we assumed that only point defects are present in the crystal and the defects are well separated from each other. It was shown by Fu et al that Fe divacancy binding energy in FeAl is 
equal $0.57 \mathrm{eV}$ meaning that there is a strong tendency of the vacancies clustering. The same is true for $\mathrm{Fe}_{3} \mathrm{Al}$ were the divacancy binding energy is equal to $1.2 \mathrm{eV}$. Such vacancies can be annealed out to open structure defects such as dislocations, grain boundaries. This can explain the obtained disagreement between the calculated defect concentrations and those obtained experimentally.

c) Elastic constants The elastic constants of $\mathrm{FeAl}$ and $\mathrm{Fe}_{3} \mathrm{Al}$ were fitted to ab initio calculations given in Table 4.1. Therefore, at zero temperature the calculated elastic constants within ab intio method and many-body atomistic potential are the same. As mentioned in Chapter 4, ab initio calculations do not include the relaxation of the atoms after putting the strain on the lattice as well as they neglect the effect of lattice vibrations present in the crystal at nonzero temperatures. Using molecular dynamics technique we studied the effect of these two factors on elastic constants of $\mathrm{FeAl}$ and $\mathrm{Fe}_{3} \mathrm{Al}$ at room temperature. As seen from the results shown in Table 5.10 the elastic constants change around $15 \mathrm{GPa}$ that is indeed bigger than the

Table 5.10 Elastic constants in $\mathrm{GPa}$ of $\mathrm{FeAl}$ and $\mathrm{Fe}_{3} \mathrm{Al}$ at different temperatures.

\begin{tabular}{|cc|c|c|c|}
\hline & & $\mathrm{C}_{11}$ & $\mathrm{C}_{12}$ & $\mathrm{C}_{44}$ \\
\hline FeAl & 0K & 281 & 140 & 167 \\
& $293 \mathrm{~K}$ & 275 & 131 & 154 \\
& Exp. $^{\mathrm{a}}$ & 181 & 114 & 127 \\
\hline $\mathrm{Fe}_{3} \mathrm{Al}$ & 0K & 272 & 210 & 196 \\
& $293 \mathrm{~K}$ & 256 & 216 & 182 \\
& Exp. & 171 & 131 & 132 \\
\hline
\end{tabular}

\footnotetext{
${ }^{a}$ Reference 25

${ }^{b}$ Reference 26
} 
change produced by the lattice vibrations included in the Debye model in Chapter 4. However, the calculated elastic constants are still significantly higher than the experimental values indicating the problem in LDA approximation of overestimating the bonding in transition metals.

c) Phonon spectra of $\mathrm{FeAl}$ and $\mathrm{Fe} \underline{3}$ Al. In the quasi-harmonic approximation the phonon frequencies of a crystal can be found by solving the following secular equation:

$$
M \omega^{2} \mathcal{E}=D(k) \mathcal{E},
$$

where $\omega$ are the phonon frequencies, $\varepsilon$ is the phonon polarization vector and $D(k)$ is the dynamical matrix of dimension $3 N \times 3 N$ where that $N$ is the number of atoms in the unit cell. Each element of the dynamical matrix represents a second derivative of the crystal total energy with respect to the atomic positions:

$$
D(k) \alpha i, \beta j=\sum_{R} \frac{\partial^{2} E}{\partial r_{\alpha i} \partial r_{\beta j}} e^{-i k \cdot R}
$$

where indexes $\alpha$ and $\beta$ correspond to the number of the atom in the unit cell, indexes $i$ and $j$ represent the corresponding cartesian projection and the summation on then right-hand site of the equation is carried over all the unit cells with the translation vector $R$. The dynamical matrix elements were evaluated numerically as the first derivative of the forces $\beta j$ with respect to the small displacements $\varepsilon$ of the reference atom $\alpha$ along all possible directions $i$ according to the equation:

$$
\frac{\partial^{2} E}{\partial r_{\alpha i} \partial r_{\beta j}}=\frac{f_{\beta j}\left(r_{\alpha i}+\varepsilon\right)-f_{\beta j}}{\varepsilon}
$$


where $\varepsilon$ in general is less than $1 \%$ of the interatomic distance. To account for anharmonic effects the dynamical matrix was calculated at several values of $\varepsilon$.displacements. It was observed that the phonon frequencies do not depend on the amplitude of the atomic displacement.

For pure iron-aluminides we calculated the phonon frequencies along the lines of the high symmetry in the Brillouin zone. The Brillouin zone for FeAl B2 structure and $\mathrm{Fe}_{3} \mathrm{Al}^{\mathrm{DO}_{3}}$ structure are shown in Figures $5.6(\mathrm{a}, \mathrm{b})$, respectively (look at the next page).

The obtained phonon frequencies of $\mathrm{FeAl}$ and $\mathrm{Fe}_{3} \mathrm{Al}$ are shown in Figure 5.7. For FeAl having two atoms per unit cell, there are six phonon modes, while in case of $\mathrm{Fe}_{3} \mathrm{Al}$ the number of the phonon modes increases to twelve. In the case of both iron-aluminides there is a high frequency part of phonon spectrum that originates primarily from the low-mass $\mathrm{Al}$ atoms and sharply separated the low-frequency pattern, which is the result of heavy-mass Fe atoms oscillations.
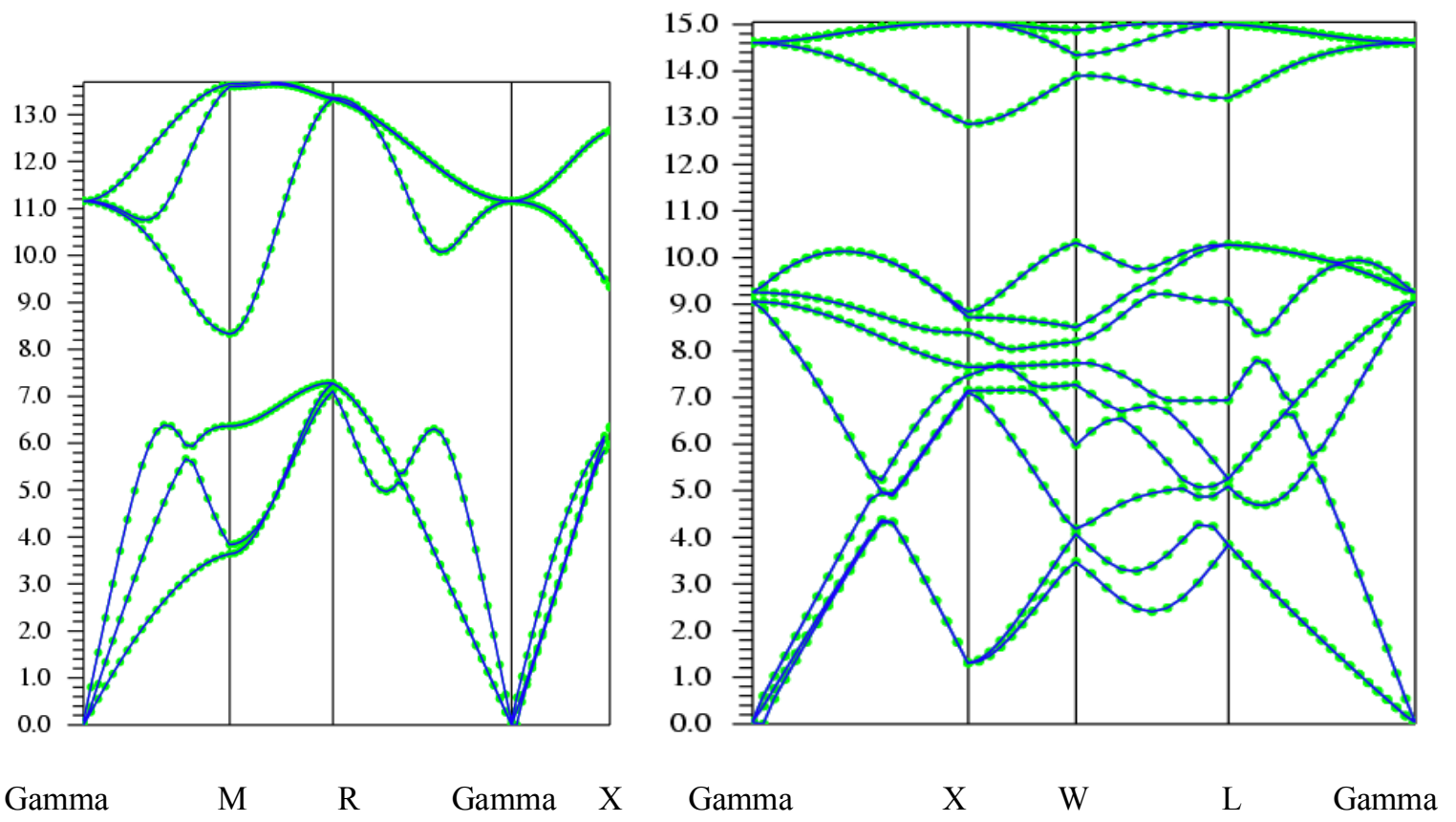

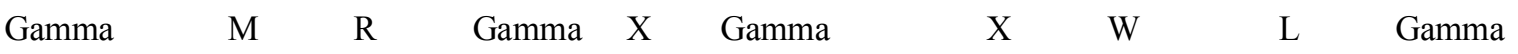

Figure 5.7 The phonon frequencies of $\mathrm{FeAl}$ ( on the left-hand side) and of $\mathrm{Fe}_{3} \mathrm{Al}$ (on the righthand side). 
Figure 5.6 The Brilloin zones of $\mathrm{FeAl} \mathrm{B}$ and $\mathrm{Fe}_{3} \mathrm{Al} \mathrm{DO}_{3}$ structures with the special k-points. The $\mathrm{B} 2$ structure has a cubic symmetry, while $\mathrm{DO}_{3}$ structure has $f c c$.

(a) $\mathbf{B}_{2}$

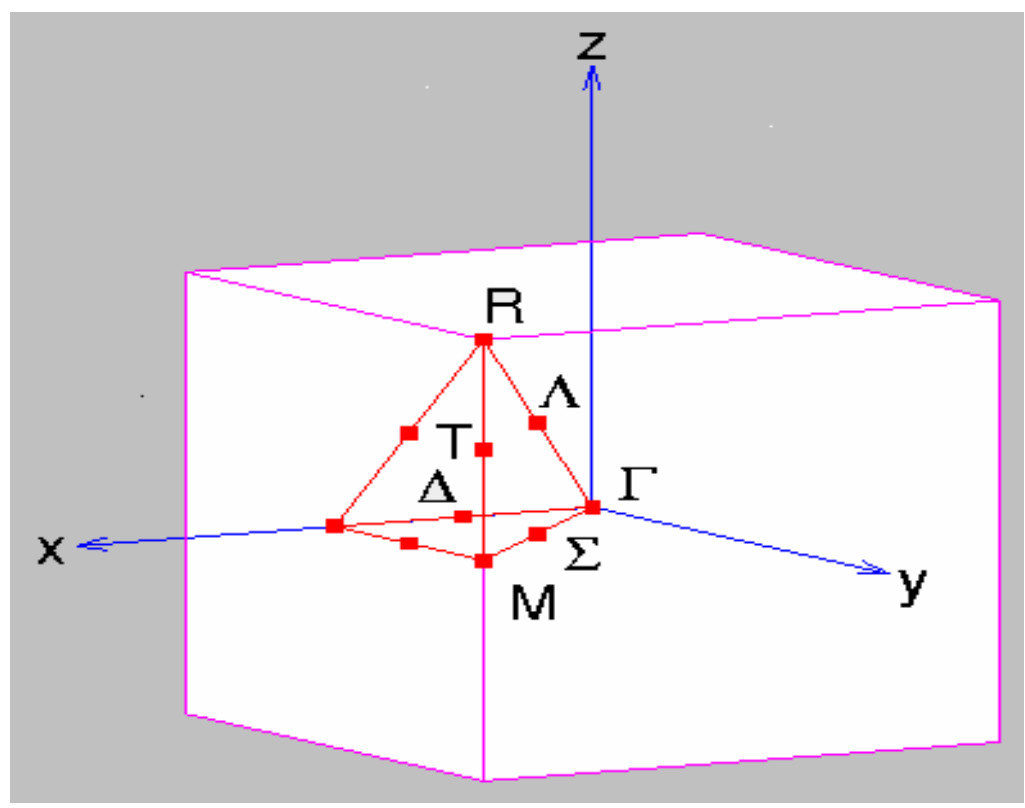

(b) $\mathrm{DO}_{3}$

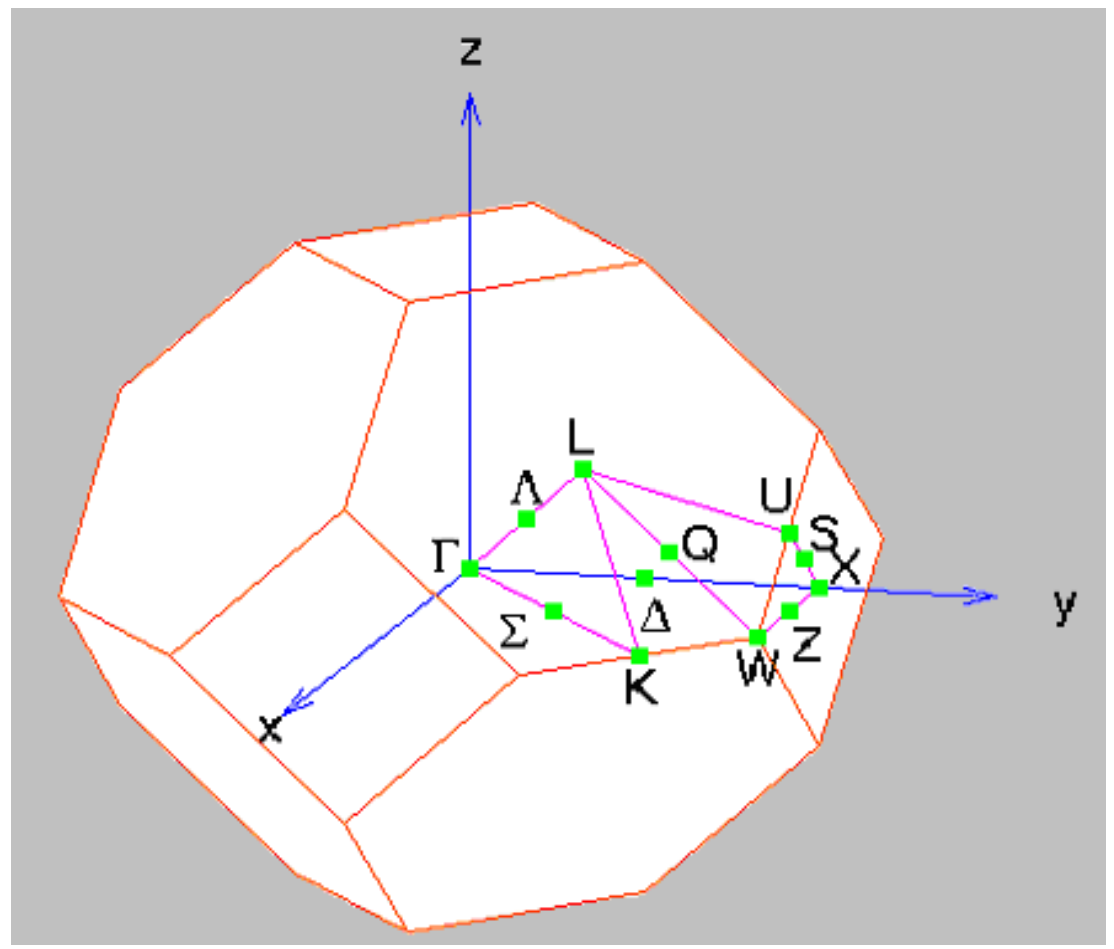


d) Linear Thermal Expansion of $\mathrm{FeAl}$ and $\mathrm{Fe}_{3} \mathrm{Al}$. The linear thermal expansions of pure iron-aluminides were calculated by two different methods: using the Debye model of a solid and by molecular dynamics simulation technique. The obtained results for $\mathrm{Fe} A l$ and $\mathrm{Fe}_{3} \mathrm{Al}$ are shown in Figures 5.8 and 5.9, respectively. For both iron-aluminides we obtained a good agreement between MD simulations and the Debye model. The difference becomes noticeable only at high temperatures due to the unharmonic effects not included in the Debye model and the optical phonons substituted by the acoustical modes. For both $\mathrm{FeAl}$ and $\mathrm{Fe}_{3} \mathrm{Al}$ the thermal expansion obtained by MD simulations is lower than the thermal expansion calculated within the Debye model, since the contribution of the optical modes into the thermal expansion is generally smaller than the contribution of the acoustical modes.

For $\mathrm{Fe}_{3} \mathrm{Al}$ we obtained a pretty good agreement between theory and experiment up to the temperature $600 \mathrm{~K}$. At the temperature $830 \mathrm{~K}$ a second-order transition from $\mathrm{DO}_{3}$ to $\mathrm{B}_{2}$ structure takes place which gradually starts at much lower temperatures. This explains the increasing divergence of calculated and experimental thermal expansion curves, since in our simulations we considered a crystal to be in a perfectly ordered $\mathrm{DO}_{3}$ structure. For FeAl the calculated thermal expansion is significantly lower than the one measured experimentally. Thus, at room temperature the calculated coefficient of thermal expansion $\left(\sim 11.7 \times 10^{-6} \mathrm{~K}^{-1}\right)$ differs $\sim 32 \%$ from its experimental value $\left(\sim 17.3 \times 10^{-6} \mathrm{~K}^{-1}\right)$. One of the reasons for that lies in overestimating the elastic constants of iron-aluminides in the LDA approximation.

The obtained coefficients of thermal expansion of $\mathrm{FeAl}$ and $\mathrm{Fe}_{3} \mathrm{Al}$ have very little temperature dependence that is in the agreement with the experiment. 
Figure 5.8 Linear Thermal Expansion of FeAl. The solid line with the circles represents the results of MD simulation, the dashed line shows the thermal expansion obtained within the Debye model and triangles are used to denote the experimentally measured thermal expansion Ref.28.

\section{FeAl}

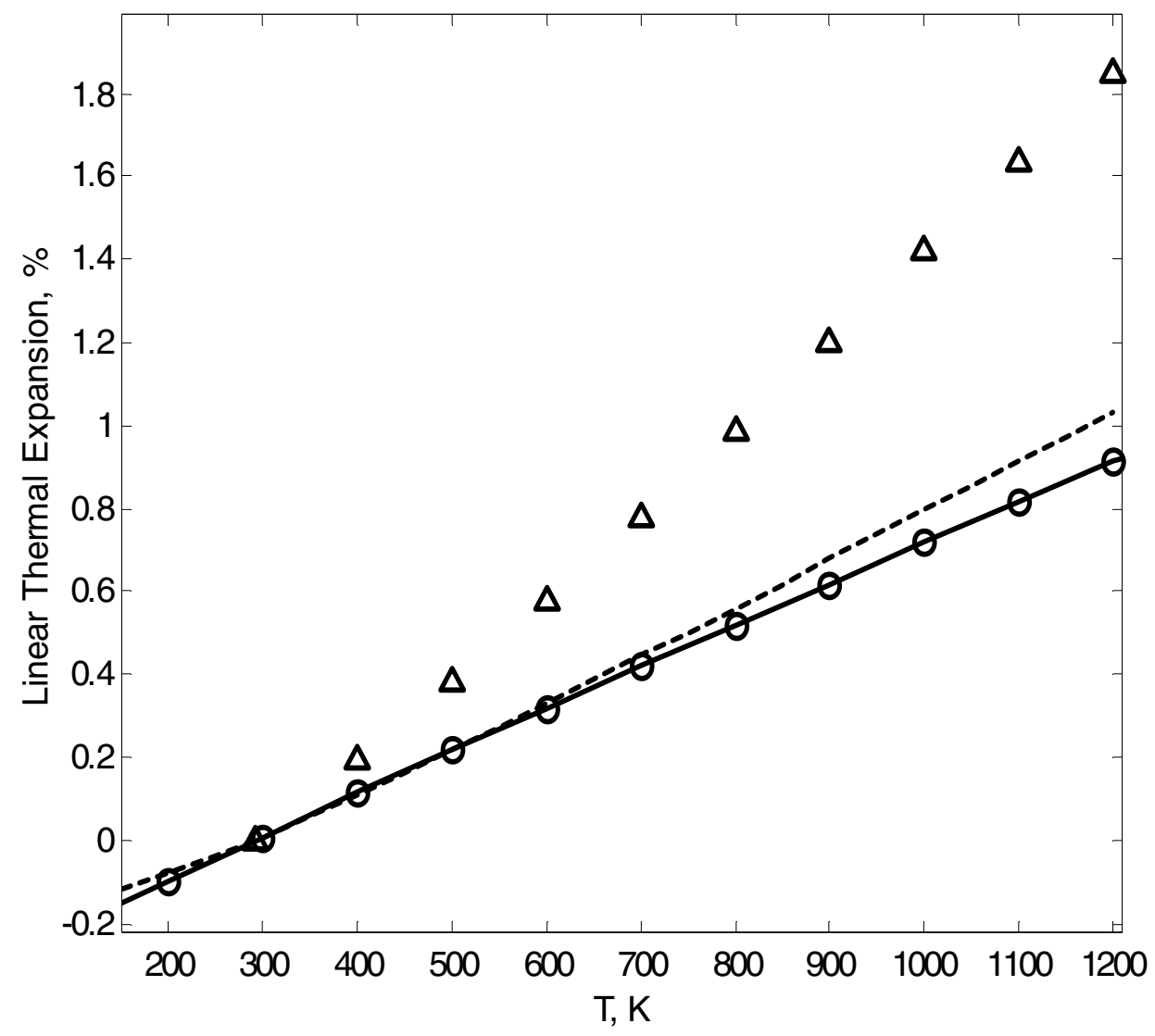


Figure 5.9 Linear Thermal Expansion of $\mathrm{Fe}_{3} \mathrm{Al}$. The solid line with the circles represents the results of MD simulation, the dashed line shows the thermal expansion obtained within the Debye model and triangles are used to denote the experimentally measured thermal expansion Ref.28.

\section{$\mathrm{Fe}_{3} \mathrm{Al}$}

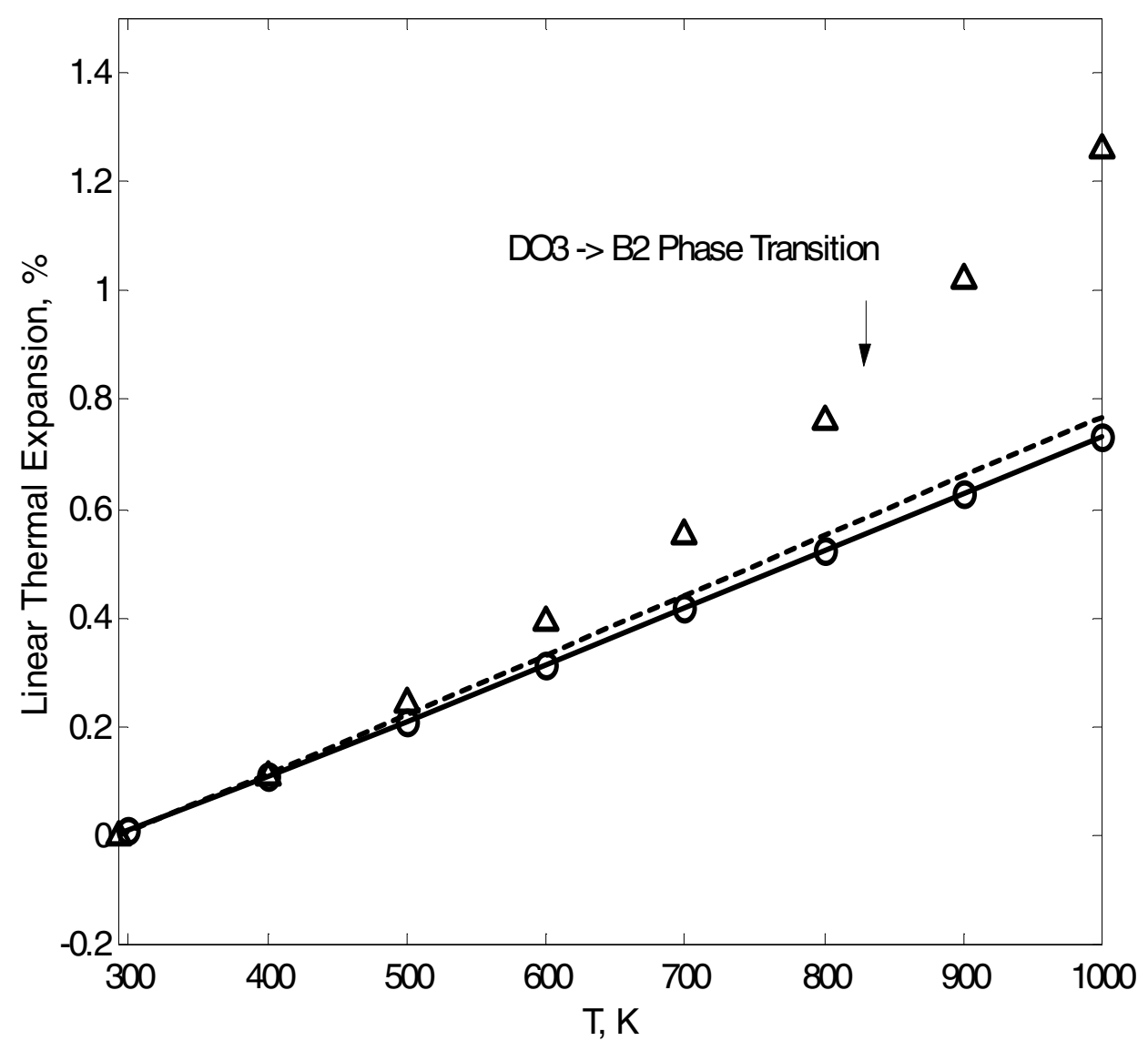




\subsubsection{Pure metals}

Thermal expansion of elemental metals. To test our potentials, we calculated the thermal expansion of elemental metals using MD simulations. The comparison between the calculated linear thermal expansion of $\mathrm{Fe}, \mathrm{Al}, \mathrm{Mo}$ and $\mathrm{V}$ are presented in Figures $5.10(\mathrm{a}, \mathrm{b}, \mathrm{c}, \mathrm{d})$, respectively. For Fe and V we obtained a very good agreement for the temperature range 200$400 \mathrm{~K}$. At temperatures close to zero the appeared disagreement is due to the quantummechanical effects not included in MD calculations. The MD method is based on solving the classical equations of motion and therefore, at zero temperatures the atomic velocities are equal to zero. However, in a real crystal the atomic vibrations do not disappear even at zero temperature, because the uncertainty principle $(\Delta x \Delta p \geq \hbar)$ requires localized ions to have a nonvanishing momentum. This is why at close-to-zero temperatures experimentally measured thermal expansion is growing slower with temperature than the one calculated by MD method.

The biggest disagreement of thermal expansion with the experiment was obtained for Al. The coefficient of thermal expansion of $\mathrm{Al}$ decreases with temperature indicating the instability of $\mathrm{Al} f c c$ structure. Since the energies of only $\mathrm{Al} b c c$ structure were included in fitting procedure of the atomistic potentials, the obtained parameters fail to describe $f c c$ structure. However, we are most interested in the adequate description of $\mathrm{Al} b c c$ phase, since this is symmetry of $\mathrm{Al}$ presented in iron-aluminides. Unfortunately, there is no experimental data of aluminum thermal expansion in $b c c$ phase. Thus, the test of Al parameters with respect to the thermal expansion was incomplete. 
Figure 5.10 Linear Thermal Expansion of Fe, Al, Mo and V. The solid line with circles represents the results of MD calculations. The dashed line with triangles shows the experimental measurements from [27].

(a) $\mathrm{Fe}$

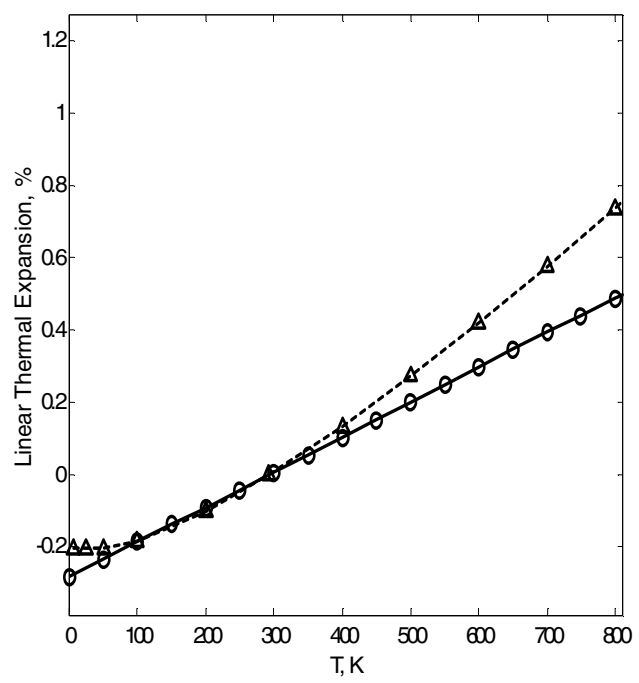

(c) $\mathrm{V}$

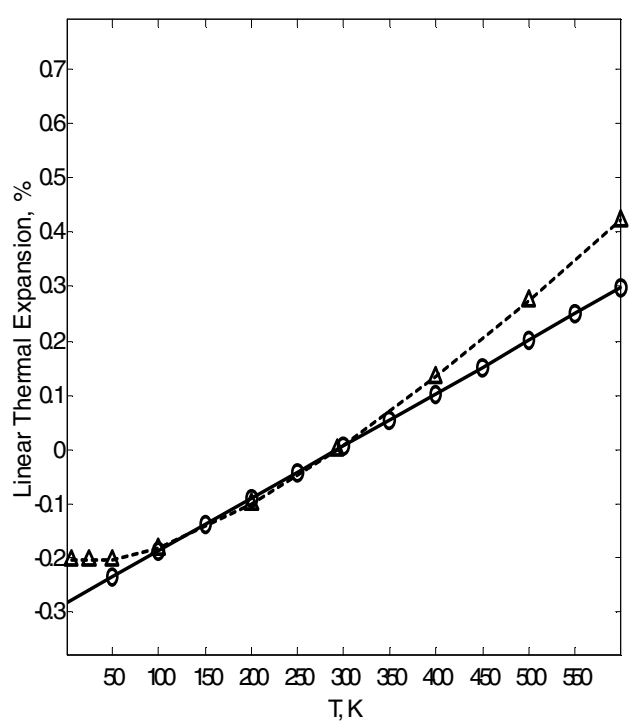

(b) Al

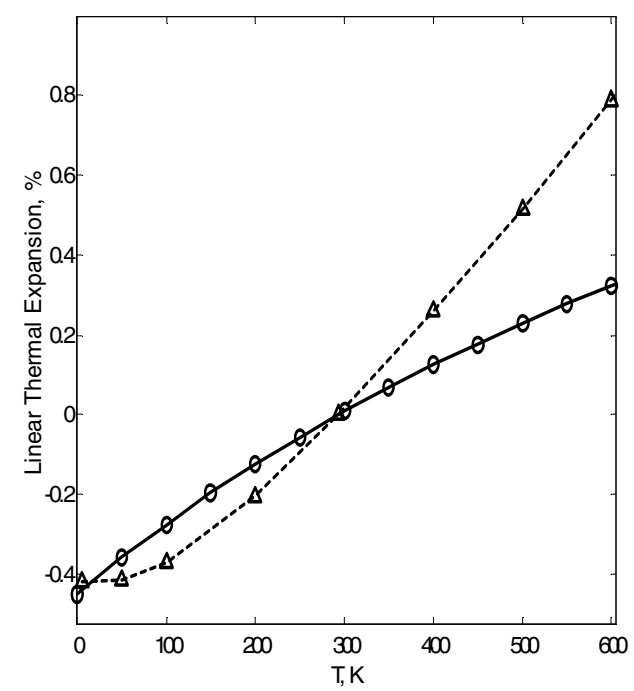

(d) Mo

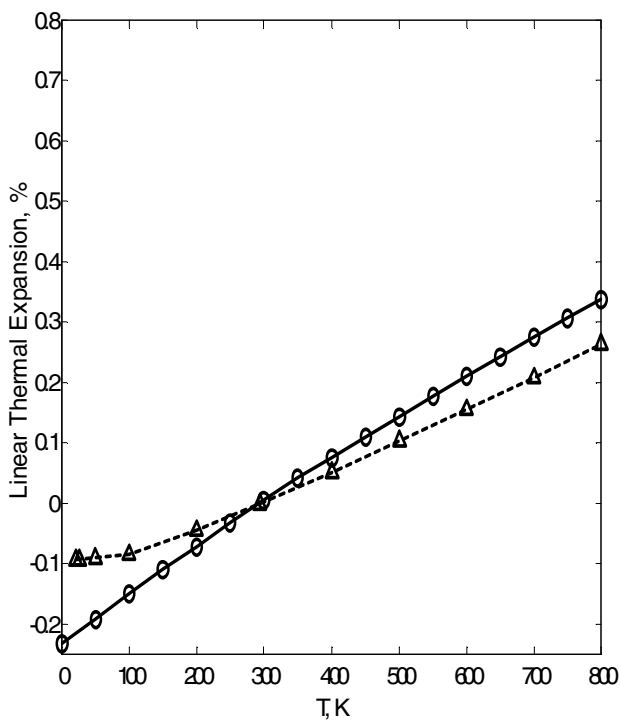




\subsubsection{Iron-aluminides with transition metal additives.}

To go beyond the calculation of thermal expansion of pure iron-aluminides, we considered the pseudo-binary alloys $\mathrm{Fe}_{1-\mathrm{x}} \mathrm{Y}_{\mathrm{x}} \mathrm{Al}$ and $\left(\mathrm{Fe}_{1-\mathrm{x}} \mathrm{Y}_{\mathrm{x}}\right)_{3} \mathrm{Al}$ with small atomic percentages $(x$ $\leq 10 \%)$ of $\mathrm{Y}(\mathrm{V}, \mathrm{Mo})$ atoms.

Since for pure iron-aluminides the thermal expansion can be easily simulated using the Debye model, we decided to examine whether this approximation is valid for the iron-aluminides in the presence of additives. For this reason, we calculated linear thermal expansion of $\left(\mathrm{Fe}_{0.917} \mathrm{~V}_{0.083}\right)_{3} \mathrm{Al}$ compound using the Debye model of a solid and by MD simulation. In order to avoid additional errors due to the deviation of atomistic potential results from those of ab initio calculation, we used many-body atomistic potentials for Debye model and MD as well. The comparison of the obtained results given in the Figure 5.11 clearly shows that the Debye model significantly overestimates the thermal expansion that prompts to question its validity in the case of additives. The existence of the defects in a crystal lattice destroys the symmetry and causes the spread of the phonon frequencies. This phenomenon is shown in Figure 5.12. While for pure $\mathrm{Fe}_{3} \mathrm{Al}$ the phonon modes are localized having four noticeable picks at 3, 5,1 0 and $15 \mathrm{THz}$, for $\mathrm{Fe}_{3} \mathrm{Al}$ with $8.3 \%$ of Vanadium concentration the phonon frequency distribution significantly changes. Thus, the excitation of the optical modes requires much less energy now and the atomic oscillations become more disorganized resulting in the decrease of the thermal expansion. 
Figure 5.11 Linear Thermal Expansion of $\left(\mathrm{Fe}_{1-\mathrm{x}} \mathrm{V}_{\mathrm{x}}\right)_{3} \mathrm{Al}$ pseudo-binary alloy $(x=8.3 \%)$. Comparison of MD simulation results with the thermal expansion calculated within the Debye model.

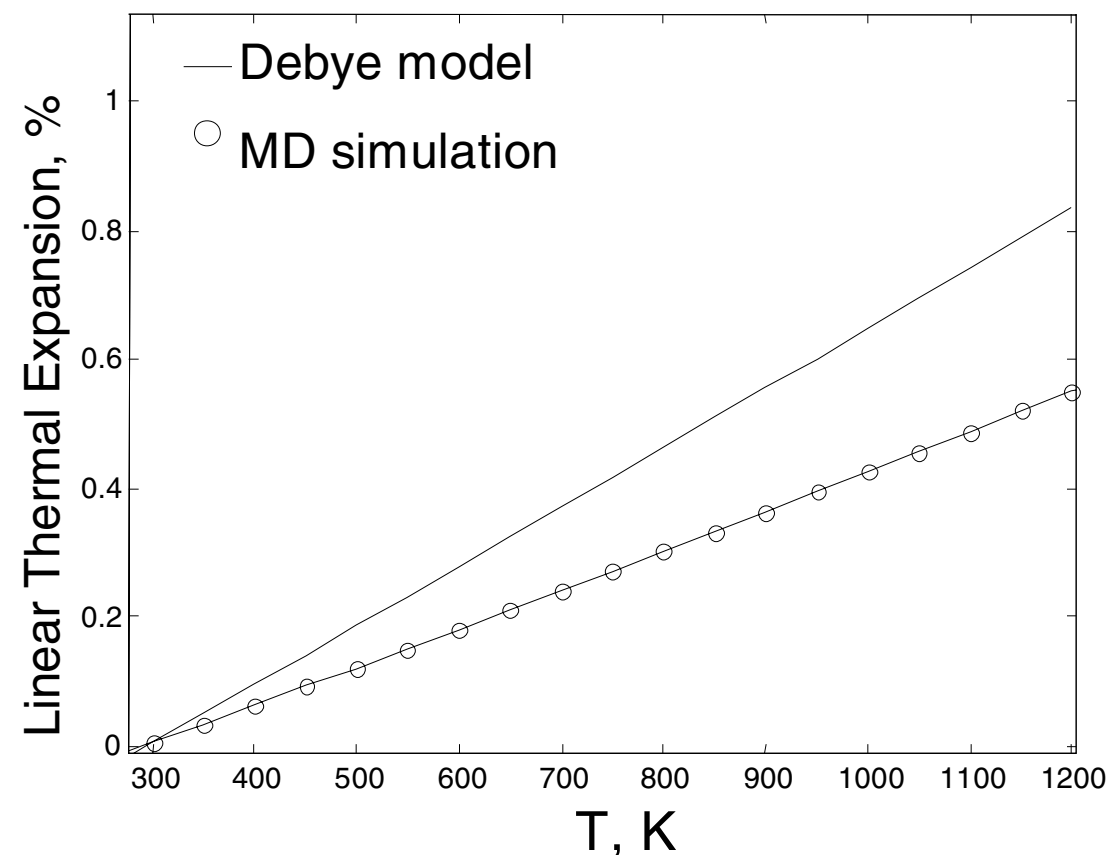

Figure 5.12 Calculated phonon density of states of $\mathrm{Fe}_{3} \mathrm{Al}$ (black line) and $\left(\mathrm{Fe}_{1-\mathrm{x}} \mathrm{V}_{\mathrm{x}}\right)_{3} \mathrm{Al}$ with $x=8.3 \%$ (redline).

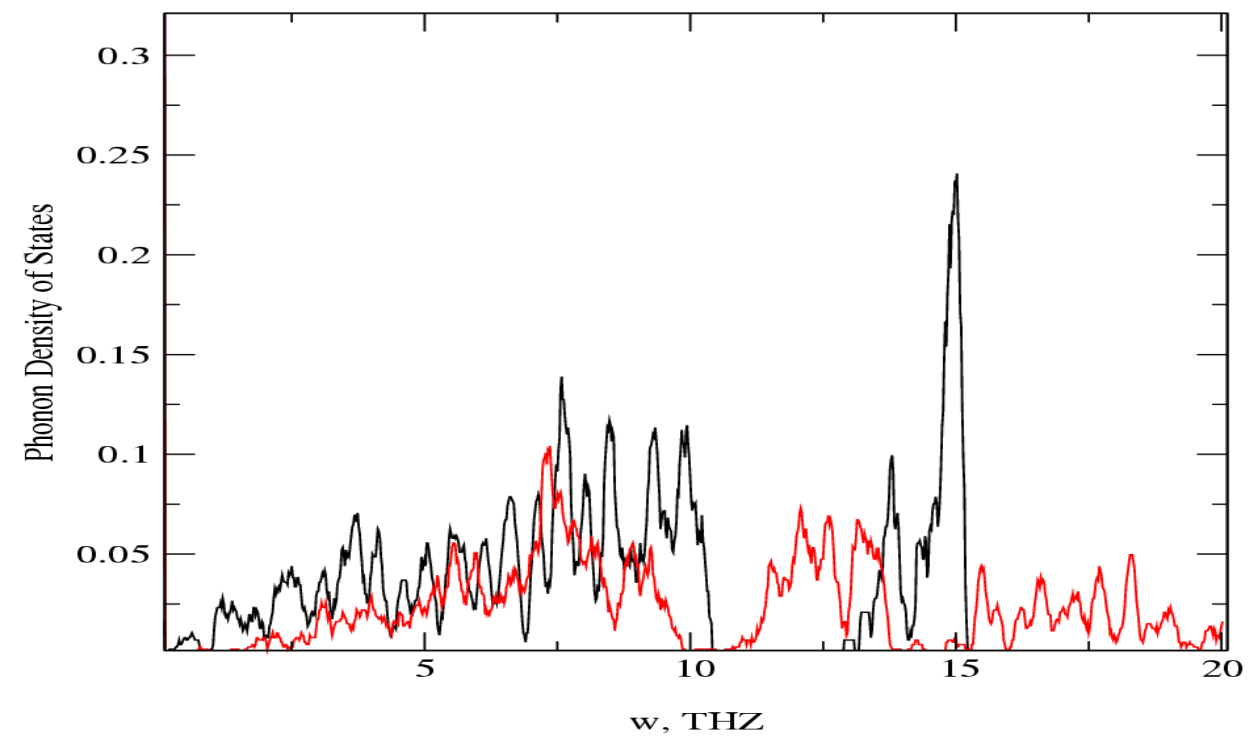


Our next step was to find the dependence of the coefficient of thermal expansion (CTE) of iron-aluminides on the additive concentration. Since in our previous ab initio calculations (Chapter 3) we determined that FeII sites energetically more favorable to be occupied by $\mathrm{V}$ and Mo atoms, the $\mathrm{V}$ and later Mo additives were randomly distributed among them. In order to confirm that additive distribution does not affect the thermal expansion, the calculations were repeated several times for each concentration. The CTEs, $\alpha$, were calculated by fitting linear thermal expansion of the compounds to third-order polynomials and taking the derivatives:

$$
\alpha=\frac{1}{\ell_{0}}\left(\frac{\partial \ell}{\partial T}\right)
$$

where $l_{0}$ is the lattice constant at room temperature. The temperature dependence of CTE is characterized by the higher than second-order derivatives of linear expansion with respect to the temperature and therefore, high accuracy is required in determining it.

The CTEs of $\mathrm{Fe}_{1-\mathrm{x}} \mathrm{V}_{\mathrm{x}} \mathrm{Al}$ pseudo-binary alloys with different concentration $x$ are shown in Figure 5.13. One can see that CTE gradually decreases with the increase of Vanadium concentration. The effect of Vanadium addition on the $\mathrm{CTE}$ of $\mathrm{Fe}_{3} \mathrm{Al}$, represented in Figure 5.14, is slightly different. Here, the temperature dependence of CTE becomes stronger with the addition of Vanadium. The effect of the transition metal additives on thermal expansion is a complicated process that results from the interplay of two factors: the modification of phonon spectrum and the change of the potential unharmonicity. Therefore, the temperature dependence of CTE can change its character with the increase of the additives. 
Figure 5.13 CTE of $\mathrm{Fe}_{1-\mathrm{x}} \mathrm{V}_{\mathrm{x}} \mathrm{Al}$ pseudo-binary alloys. The results of molecular dynamics simulation.

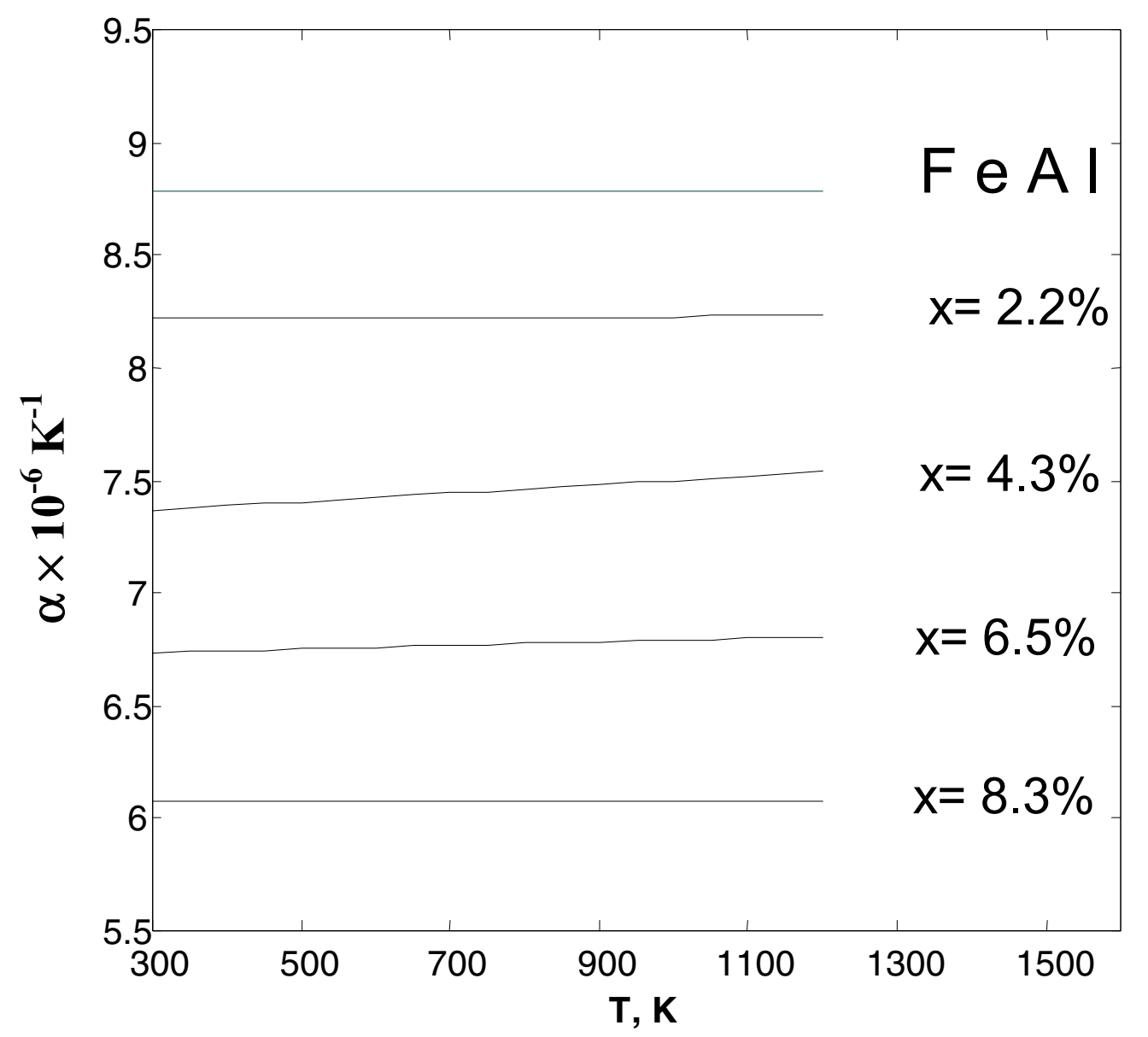


Figure 5.14 CTE of $\left(\mathrm{Fe}_{1-\mathrm{x}} \mathrm{V}_{\mathrm{x}}\right)_{3} \mathrm{Al}$ pseudo-binary alloys. The results of molecular dynamics simulation.

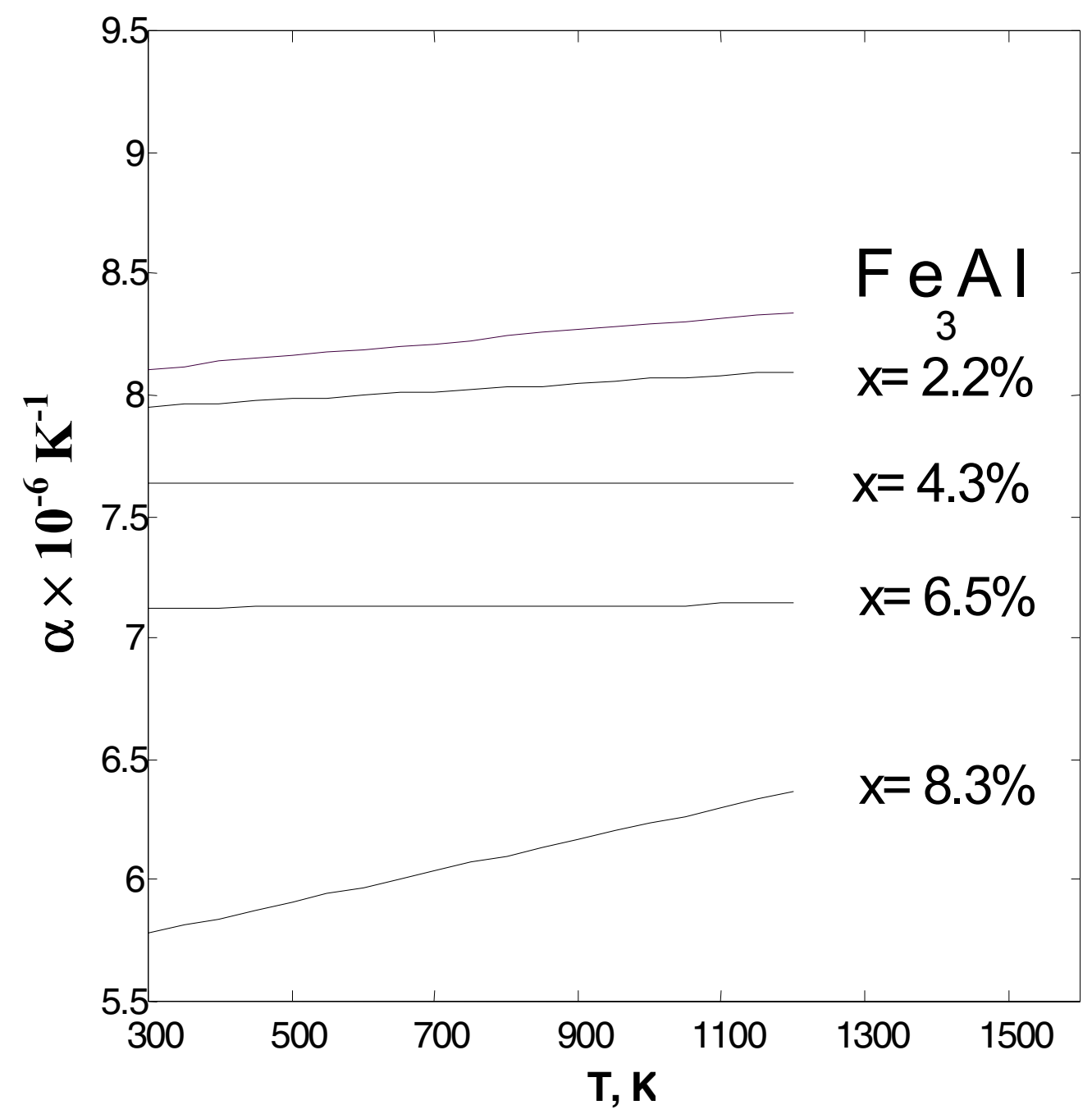


Thus, when $\mathrm{V}$ is substituted for Fe and FeAl compound the calculated CTE appears to be temperature independent for all Vanadium concentrations except 4.3\%. For $\mathrm{Fe}_{3} \mathrm{Al}$ the effect is opposite. For the concentrations 4.3 and $6.5 \%$ of Vanadium, the CTE is temperature independent. When Vanadium concentration reaches $8.3 \%$, CTE starts to increase with the temperature indicating the rising anharmonicity of the interatomic potential.

To study the effect of Mo additives, we considered only one concentration of this transition metal in $\mathrm{Fe}_{3} \mathrm{Al}$ compound, namely $4.3 \%$ of Mo substituted for the Fe content. The calculated CTE, presented in the Figure 5.15, decreases with temperature indicating the structural instability of the compound. A similar effect of Mo addition was observed in nickel binary alloys [5].

Figure 5.15 CTE of $\left(\mathrm{Fe}_{1-\mathrm{x}} \mathrm{Mo}_{\mathrm{x}}\right)_{3} \mathrm{Al}$ pseudo-binary alloys. The results of $\mathrm{MD}$ simulation.

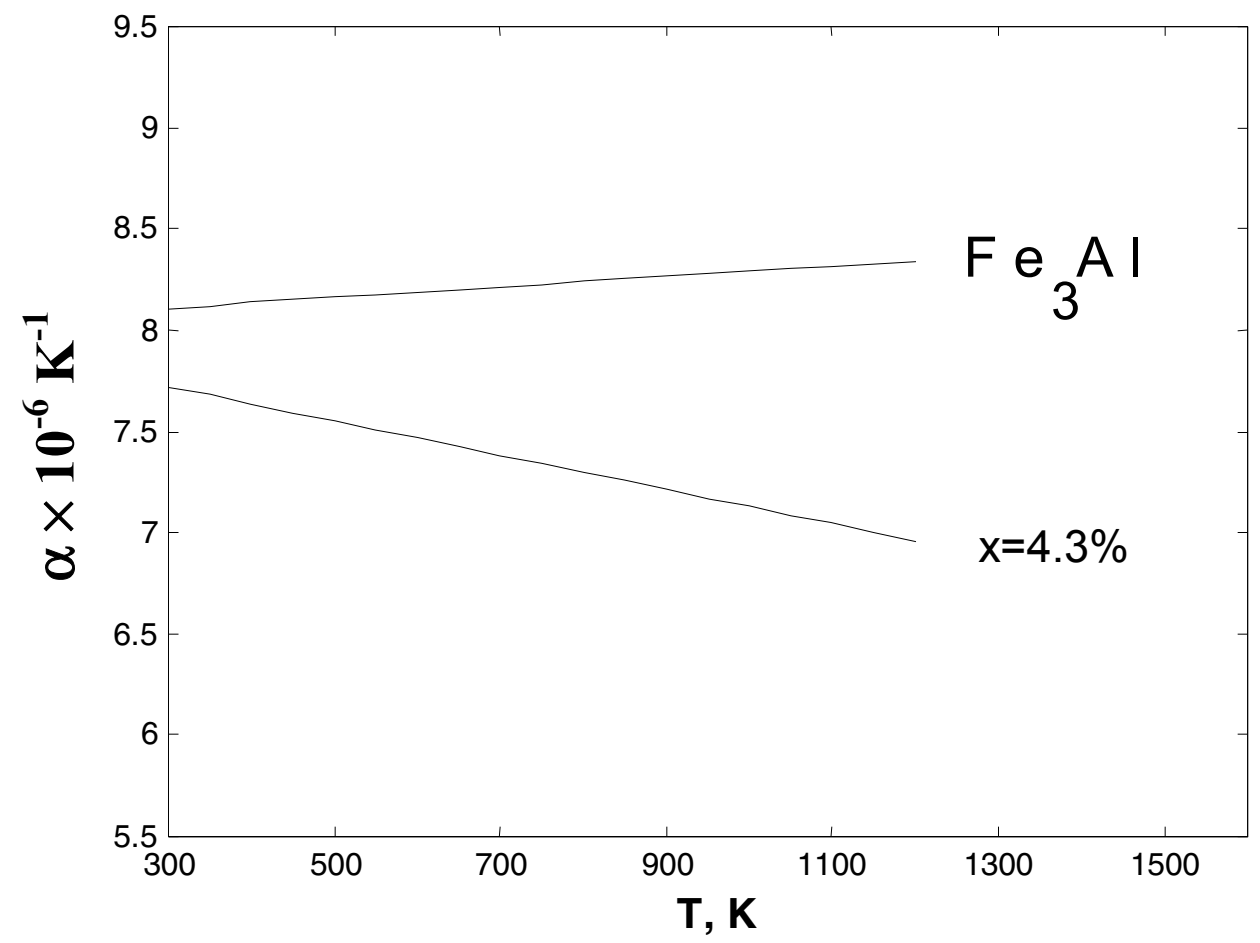




\subsection{Conclusions}

We obtained ab initio based many-body atomistic potentials that correctly describe ironaluminum compounds at two different compositions: $\mathrm{FeAl}$ and $\mathrm{Fe}_{3} \mathrm{Al}$. The calculated lattice parameters, defect formation energies, elastic constants, and thermal expasion are all consistent with $a b$ initio calculations. The deviation of the elastic constants and, consequently, thermal expansion from the experimental results is mostly referred to the LDA overestimating the strength of the interatomic bonding. For pure iron-aluminides the calculated thermal expansion within the Debye model is found to be in a good agreement with molecular dynamics simulation. This fact confirms that the Debye model serves as an appropriate approximation for these intermetallic compounds.

When the transition metal additives, $\mathrm{V}$ and $\mathrm{Mo}$, are present in the iron-aluminum system, the symmetry of a crystal gets broken therefore strongly modifying the phonon spectrum of the material. Here, the Debye model is no longer applicable and we used MD simulation to study the dependence of the coefficient of thermal expansion (CTE) on the additive concentrations. We found that addition of $\mathrm{V}$ significantly decreases CTEs of both $\mathrm{FeAl}$ and $\mathrm{Fe}_{3} \mathrm{Al}$, while at Mo concentration 4.3\%, CTE of $\mathrm{Fe}_{3} \mathrm{Al}$ compound decreases with temperature indicating the appearance of instability of $\mathrm{DO}_{3}$ structure.

The obtained many-body atomistic potentials can be further applied in studying the effect of Mo and $\mathrm{V}$ additives on the mechanical properties of iron-aluminides and simulation of the extended defects such as dislocations and grain-boundaries. 


\section{References}

1. R.Besson, J.Morillo, Phys.Rev. B, vol.55, 1 (1997), pp.193-204

2. Wen-qung Zhang, Qian Xie, Xi-jin Ge, J.Appl.Phys. 82(2), 1997

3. J.H.Rose, J.R.Smith, F.Guinea, J.Ferrante, Phys.Rev.B, vol.29, 6 (1984)

4. T.B.Massalski, Ed., Binary Alloy Phase Diagrams, vol.1\&2, American Society of Metals, 1986

5. J.Mei, B.R.Cooper, Y.G.Hao, F.L.Van Scoy, Alloy Modeling and Design, Edited by G.M.Stocks and P.E.A.Turchi, (The Minerals, Metals \& Materials Society, 1994)

6. J.Mei, B.R.Cooper, S.P.Lim, Phys.Rev. B, vol.54, 1, p.178-183 (1996)

7. J.A.Moriarty, Phys.Rev. B, vol.38, 3199 (1988)

8. J. M. Wills, O. Eriksson, M. Alouani, and D. L. Price, Full-Potential LMTO total energy and force calculation, Electronic Structure and Physics Properties of Solids, H. Dreysse(Ed.), Springer-Verlag, Berlin, 2000, pp 148-167.

9. M.P.Allen, D.J.Tildesley, Computer Simulation of Liquids, (Oxford 1994)

10. H.C.Andersen, Molecular dynamics simulation at constant pressure and/or temperature, J.Chem.Phys., vol.72, 2384-93 (1980)

11. M.S.Daw and M.I.Baskes, Phys.Rev.Lett.50, 1285 (1983)

12. M.I.Baskes, J.S.Nelson, A.F.Wright, Phys.Rev. B, vol.40, 6085 (1989)

13. M.I.Haftel, M.Rosen, Phys.Rev. B, vol.64, 19 (2001)

14. W.H.Press et al, Numerical Recipes: the art of scientific computing, $2^{\text {nd }}$ ed., (Cambridge University Press, 1992) 
15. A.E.Carlson and P.E.Mechter, Intermetallic Compounds: Principles nad Practice, edited by J.H.Westbrook and R.L.Fleischer (Wiley, New Yok, 1994)

16. F.Lechermann, F.Welsch, C.Eslasser, C.Ederer, M.Fahnle, J.M.Sanchez, B.Meyer, Phys. Rev. B, vol.65, 2002, p.132104-1 - 4

17. C.L.Fu, M.H.Yoo, Acta metal.mater., vol.40, No.4, pp.703-711, 1992

18. J.Rieu, C.Goux, Mem.Scient.Rev.Metall, vol.66, 869 (1969)

19. L.S.Muratov, B.R.Cooper, J.M.Wills, Mat.Res.Soc.Symp. Proc. Vol.538, 1999 Material Research Society

20. I.Baker, P.R.Munroe, High Temperature Aluminides \& Intermetallics, edited by S.H.Wang, C.T.Liu, D.P.Pope, J.O.Stiegler, (The Minerals, Metals \& Materials Society, 1990), p.425

21. D.Weber, M.Meurtin, D.Paris, A.Fourdeux, P.Lesbats, J.Phys. C, vol. 7, 332 (1997)

22. Y.A.Chang, L.M.Pike, C.T.Liu, A.R.Bilbrey, D.S.Stone, J.Intermetall. vol.1, 107 (1993)

23. H.Lafi, M.Dirand, L.Bourden, J.Hertz, D.Weber, P.Lesbats, Acta. Metall., vol.34, 425 (1986)

24. G.Simmons, H.Wang, Single Crystal Elastic Constants and Calculated Aggregate Properties (M.I.T.Press, Boston, 1971)

25. H.J.Leamy, E.D.Gibson, F.X.Kayser, Acta Metalurgica vol.15, December 1967, pp.18271838

26. B.Meyer, V.Scott, M.Fahnle, Phys. Rev. B 58, 22 (1998)

27. Y.S.Touloukian, R.K.Kirby, R.E.Taylor, P.D.Desai, Thermodynamical Properties of matter: Thermal Expansion Metallic Elements and Alloys, ( IFI/ Plenum, NY 1975) 
Tatiana S. Seletskaia

Home:

e-mail: tseletsk@mix.wvu.edu

39 North $15^{\text {th }}$ Street

Indiana, PA15701

(724)-349-6549
Work:

Physics Department, PO Box 6315

Morgantown, WV 26506-6315

(304)-293-3422 x 1448

\section{OBJECTIVE:}

A position providing the opportunities to further develop and apply skills and experience in the areas of professional interest

PROFESSIONAL INTEREST:

Application of theoretical models to realistic system simulations

\section{EDUCATION:}

- Ph.D.(Physics) - October, 2002

Physics Department, West Virginia University (GPA 3.71)

- M.S. (Physics) - August, 1999

Physics Department, West Virginia University

- September, 1995 - June, 1996

TEMPUS scholarship, Studies at Manchester University, United Kingdom

- B.S. (Physics) - June, 1996

Department of Physics and Mathematics, Latvian University, Latvia

\section{WORK EXPERIENCE:}

- June, 1998 - Present

Graduate Research Assistant, Supervisor Dr.B.Cooper

Physics Department, West Virginia University, Morgantown, WV

Project: Calculation of the Thermal Expansion Coefficients of

$\mathrm{Fe}_{3} \mathrm{Al}$ and FeAl with the Addition of Transition Elements.

- August, 1997 - May, 1998

Teaching Assistant

Physics Department, West Virginia University, Morgantown, WV

PROFESSIONAL SERVICE AND MEMBERSHIPS: Member of American Physical Society CURRENT RESEARCH INTERESTS:

- Density-functional theory in the local density approximation

- Ab-initio and ab-initio based calculations of transition metal systems and structural materials

- Application of semi-empirical methods such as tight-binding and Embedded Atom Method in large-scale simulations

- Calculations of thermal properties and solid state phase stability of structural materials

\section{COMPUTER EXPERIENCE:}

- Operating Systems:

- Languages:

- Parallel Programming: MPI library

- Numerical Methods:

PERSONAL INTERESTS: Languages: Russian, French, Latvian 